\title{
THERMAL PROPERTIES OF YTTRIUM ALUMINUM GARNETT FROM MOLECULAR DYNAMICS SIMULATIONS
}

\author{
by \\ Majid S. alDosari \\ Thesis \\ Submitted to the Faculty of the \\ Graduate School of Vanderbilt University \\ in fulfillment of the requirements \\ for the degree of \\ MASTER OF SCIENCE \\ in \\ Mechanical Engineering
}

May, 2012

Nashville, Tennessee

APPROVED:

Professor Greg Walker, Chair

Professor Deyu Li

Professor Jason Valentine 


\begin{abstract}
AKNOWLEDGEMENTS
Acknowledgment is mainly to Saudi ARAMCO and its Advanced Degree Program which sponsored my degree. Also, every Thermal Engineering Lab member has contributed in some way to this work. But, it is difficult to order their contribution so they are listed here according to their seating in counter-clockwise order starting from the lab's only window: Nicholas Roberts, Terry Musho, Ebonee Walker, Mike Myers, and Rachel Hansel. In addition, I'd like to acknowledge the Advanced Computing Center for Research and Engineering (ACCRE) at Vanderbilt for providing the necessary computational resources. Last but not least, I appreciate Dr. Walker's direction in this work which I have found to be interesting, enjoyable, and satisfying.
\end{abstract}


LIST OF FIGURES $\ldots \ldots \ldots \ldots \ldots \ldots \ldots \ldots \ldots \ldots \ldots \ldots \ldots \ldots \ldots, \mathrm{v}$

LIST OF TABLES $\ldots \ldots \ldots \ldots \ldots \ldots \ldots \ldots \ldots \ldots \ldots \ldots \ldots \ldots \ldots \ldots \ldots \ldots$ vi

NOMENCLATURE $\ldots \ldots \ldots \ldots \ldots \ldots \ldots \ldots \ldots \ldots \ldots \ldots \ldots \ldots \ldots$ vii

Chapter

I. INTRODUCTION $\ldots \ldots \ldots \ldots \ldots \ldots \ldots \ldots \ldots \ldots \ldots \ldots \ldots \ldots, 1$

II. YAG MODEL $\ldots \ldots \ldots \ldots \ldots \ldots \ldots \ldots \ldots \ldots \ldots \ldots \ldots \ldots \ldots, 4$

III. LATTICE CONSTANT, SPECIFIC HEAT CAPACITY, THERMAL

EXPANSION $\ldots \ldots \ldots \ldots \ldots \ldots \ldots \ldots \ldots \ldots \ldots \ldots \ldots \ldots \ldots \ldots \ldots \ldots$

1. Simulation Setup. .............................. 11

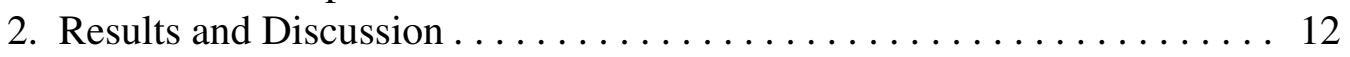

IV. MELTING TEMPERATURE......................... 15

1. Results and Discussion ........................ 17

V. THERMAL CONDUCTIVITY FROM NON-EQUILIBRIUM SIMULATIONS $\ldots \ldots \ldots \ldots \ldots \ldots \ldots \ldots \ldots \ldots \ldots \ldots \ldots \ldots \ldots .21$

1. Simulation Setup.............................. 22

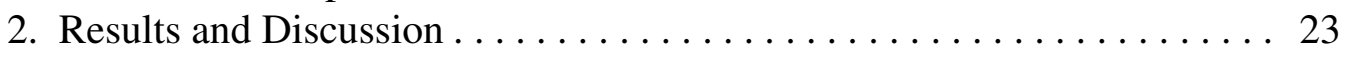

VI. COnCLUSIONS $\ldots \ldots \ldots \ldots \ldots \ldots \ldots \ldots \ldots \ldots \ldots \ldots \ldots \ldots \ldots$

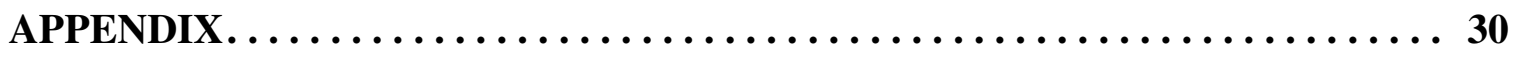


BIBLIOGRAPHY $\ldots \ldots \ldots \ldots \ldots \ldots \ldots \ldots \ldots \ldots \ldots \ldots \ldots \ldots \ldots \ldots \ldots$ 


\section{LIST OF FIGURES}

Figure 1 CONVENTIONAL UNIT CELL YAG SYMMETRY ON [1 100$]$

FACE. Red:O, Green:Al, Blue:Y. (Image generated by Jmol [1]). . . . 5

Figure 2 POLYHEDRA IN YAG [2] (with permission from the American Chemical Society) .................... 6

Figure 3 ENERGY CONSERVATION OF $2 \times 2 \times 2$ UNIT CELLS IN NVE

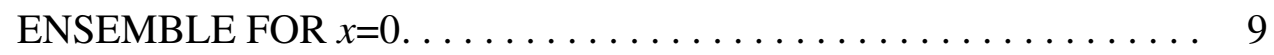

Figure 4 ENERGY CONSERVATION OF $2 \times 2 \times 2$ UNIT CELLS IN NVE

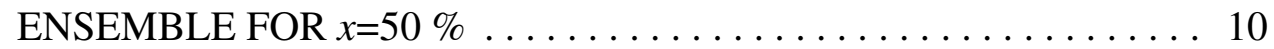

Figure 5 TEMPERATURE VS. TIME FOR 25\% Al SUBSTITUTION . . . . . 12

Figure $6 \quad c_{p}$ VS. SUBSTITUTION FRACTION, $x \ldots \ldots \ldots \ldots \ldots \ldots \ldots$

Figure 7 RADIAL DISTRIBUTION FUNCTION FOR 6 EVACUATED ALUMINUM ATOMS . . . . . . . . . . . . . . . . . . 17

Figure 8 POTENTIAL ENERGY FOR 6 EVACUATED ALUMINUM

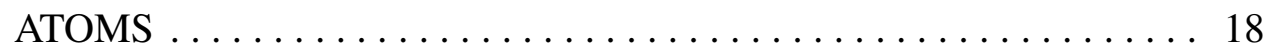

Figure 9 POTENTIAL ENERGY FOR 15 EVACUATED ALUMINUM ATOMS .............................. 19

Figure 10 LINDEMANN INDEX FOR 6 EVACUATED ALUMINUM ATOMS . 20

Figure 11 LINDEMANN INDEX FOR 15 EVACUATED ALUMINUM

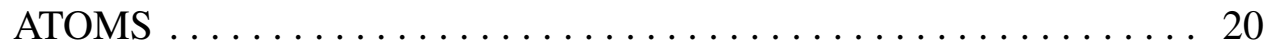

Figure 12 NON-EQUILIBRIUM MD CONFIGURATION . . . . . . . . . 22

Figure 13 AVERAGE TEMPERATURE PROFILE FOR A SAMPLE SIZE 4 UNIT CELLS WIDE, WITH 50\% Al SUBSTITUTION, $\Delta T=150$ K. . . 24

Figure 14 NON-EQUILIBRIUM THERMAL CONDUCTIVITY TRENDS SPECIFYING TEMPERATURE DIFFERENCE FOR $x=0,50$,

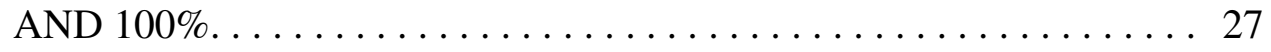

Figure 15 NON-EQUILIBRIUM THERMAL CONDUCTIVITY TRENDS SPECIFYING SPECIFYING TEMPERATURE GRADIENT FOR $x$ $=0,50$, AND $100 \% \ldots \ldots \ldots \ldots \ldots \ldots \ldots \ldots \ldots \ldots \ldots \ldots \ldots \ldots \ldots$ 


\section{LIST OF TABLES}

Table 1 THERMAL PROPERTIES CRYSTALLINE OF YAG . . . . . . . . 3

Table 2 TWO-BODY PARAMETERS .............. 5

Table $3 \quad$ THREE-BODY PARAMETERS $\ldots \ldots \ldots \ldots \ldots \ldots \ldots \ldots \ldots$

Table 4 THERMAL CONDUCTIVITY SIMULATION SETTINGS $\ldots \ldots \ldots 23$ 


\section{NOMENCLATURE}

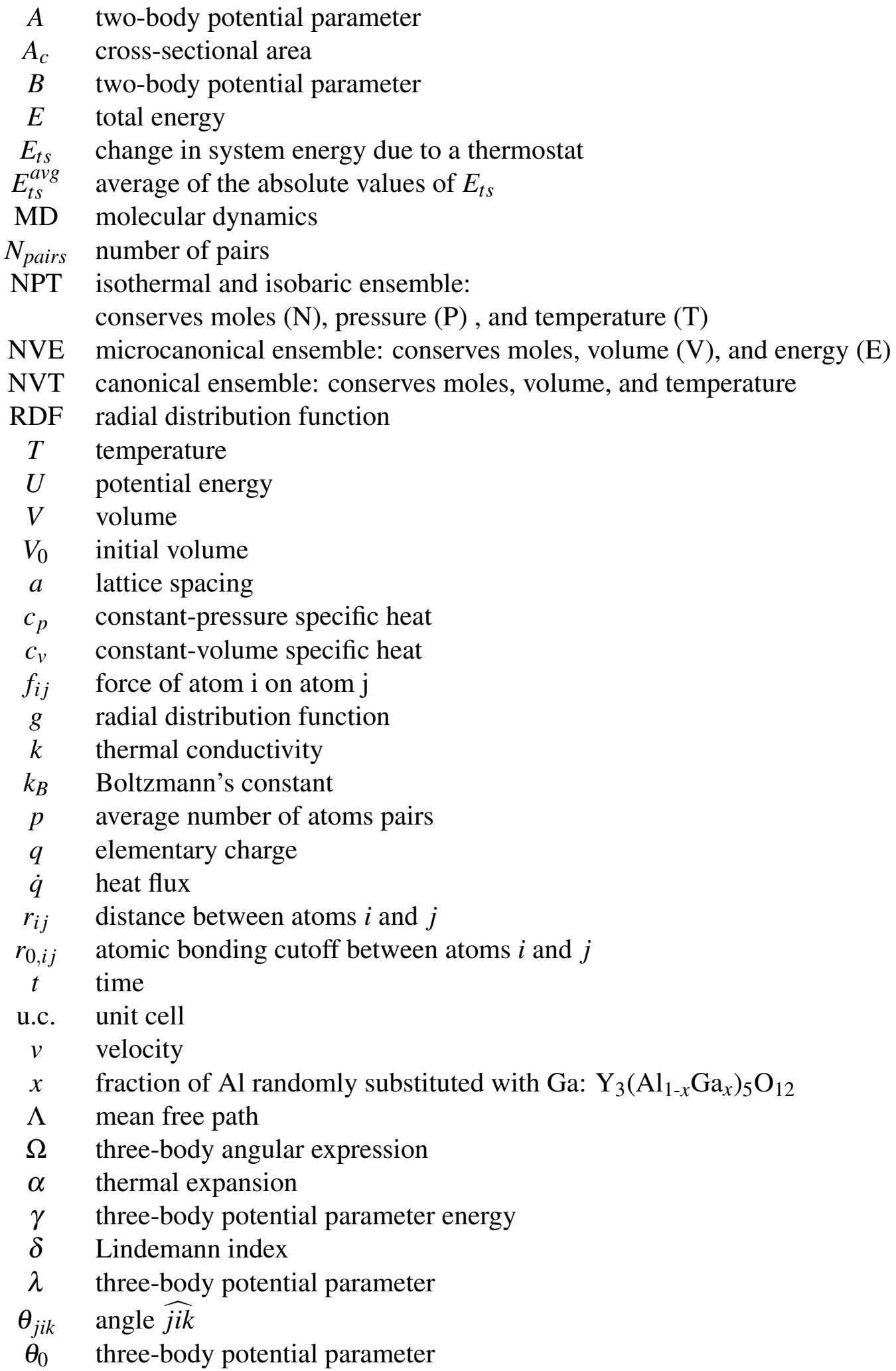




\section{CHAPTER I}

\section{INTRODUCTION}

Yttrium Aluminum Garnet ( $\mathrm{YAG}, \mathrm{Y}_{3} \mathrm{Al}_{5} \mathrm{O}_{12}$ ) is a synthetic crystal with a complicated atomic structure. Doped varieties are used for solid state lighting [3], as thermographic phosphors [4], in thermal barriers [5], and as lasing mediums [6]. The inherent thermal design issues of these applications motivate the study of the thermal transport and bulk properties in YAG. However, recent efforts to exploit and understand the small-scale behavior of YAG in, for example, radiation environments and luminescent excitation has prompted the study of the material from a atomistic point of view. In these detailed research studies quantities such as dominant vibrational modes, density of states and other non-equilibrium descriptions are important to deducing the interactions of thermal quantities with other physical effects.

In the foregoing work, a molecular dynamics simulation is used to extract bulk thermal properties of the YAG structure. By comparing the results to established bulk values, we can learn about the efficacy of the simulation approach to model thermal properties and transport of pure YAG crystals. This effort will lay the ground work for molecular dynamics in further studies of more detailed properties that can not be compared directly with measurements.

Since YAG has a relatively low thermal conductivity, its thermal transport is primarily governed by phonons, which are inferred from atomic motion. Therefore, classical molecular dynamics (MD) simulations can offer insight into the thermal properties of YAG

of varying system parameters as long as temperatures are above $50 \mathrm{~K}$, approximately $10 \%$ of the Debye temperature.

In the work introduced by Hansel [4], gallium is substituted for aluminum in vari- 
ous percentages to study its effect on luminescence. In this work, the perfect YAG structure will be defects by substituting aluminum with gallium in varying percentages. Subsequent calculation of the thermal properties will allow us to study the substitutional effects on constant-pressure specific heat, lattice constant, thermal expansion, and thermal conductivity. Realize that for these simulations, the mass of the aluminum atom will be changed to that of gallium, but the interatomic potential will remain the same as the aluminum. Therefore, we should be able to determine whether the mass of the substitution or the bonding dominates the thermal properties by examining the deviation of the simulation from expected values.

In addition to the thermal properties of substituted YAG, we will consider the melting temperature of pure YAG and extrapolation of the thermal conductivity to infinite systems for additional validation. However, molecular dynamics is notoriously poor at predicting melting temperature because melting is a process that requires localized initiation [7]. In periodic crystals used in simulations, the localized initiation does not exist; therefore, simulated melting temperatures are usually far greater than measured melting temperatures. Consequently, we will attempt to recover the melting temperature by introducing vacancies to mimic real crystals. To deduce the macroscale thermal conductivity, the system size will be systematically varied to provide adequate data for extrapolation. We also know that the extrapolation of size dependent data can be imprecise for complex systems, so we want to evaluate the efficacy of using standard extrapolation techniques to recover bulk thermal conductivity.

Thermal properties of pure, crystalline YAG are reported in Table 1 for comparison to results from our simulations. 
Table 1 THERMAL PROPERTIES CRYSTALLINE OF YAG

Melting Temperature, $\mathrm{T}_{\mathrm{m}}$

Thermal Expansion, $\alpha$

Specific Heat, $c_{p}$

Thermal Conductivity, $\mathrm{k}$

Debye Temperature, $T_{D}$
$2213 \mathrm{~K}$ [8]

$7.0 \cdot 10^{-6} \mathrm{~K}^{-1} @ 300 \mathrm{~K}[9]$

$0.6 \mathrm{~J} \cdot \mathrm{g}^{-1} \mathrm{~K}^{-1} @ 300 \mathrm{~K}$ [10] [11]

$10-14 \mathrm{~W} \cdot \mathrm{m}^{-1} \mathrm{~K}^{-1}[12]$

$750 \mathrm{~K}[13]$ 


\section{CHAPTER II}

\section{YAG MODEL}

YAG crystallizes in a highly symmetric cubic structure (detailed in Reference [2]). The conventional unit cell of YAG contains 160 atoms comprising of 24 yttrium, 40 aluminum, and 96 oxygen atoms; with a lattice parameter of $12.01 \AA$ [14]. The relative arrangement of $\mathrm{Y}, \mathrm{O}$, and $\mathrm{Al}$ can be described with polyhedra as shown in Figure 2, while the symmetry can be seen in Figure 1.

Jun, et al. [15] have modeled the complex structure of pure YAG for classical molecular dynamics simulation by fitting elasticity and lattice constant data to representative potential energy functions. We are leveraging this work to subsequently calculate thermal properties from these potentials that were designed for mechanical properties. As such, non-transport properties such as thermal expansion and specific heat should compare well. However, thermal conductivity calculations, which require precise description of the anharmonicty may deviate from an insufficiently tuned interatomic potential. Therefore the focus is to evaluate the use of the already generated potentials for thermal (both trans-

port and non-transport) properties. Moreover, we will substitute aluminum for gallium by changing only the mass of the oscillators; the interatomic potential will remain the same for the substitutional studies.

The potential energy for any pair of atoms (ions), $i$ and $j$, are given by the sum of a Coulombic and exponential term given by

$$
U_{i j}=c \frac{q_{i} q_{j}}{r_{i j}}+A_{i j} \exp \left(-\frac{r_{i j}}{B_{i j}}\right)
$$

where $c$ is an energy conversion constant equal to $14.4 \mathrm{eV} \cdot \AA \cdot e^{-1}$. 


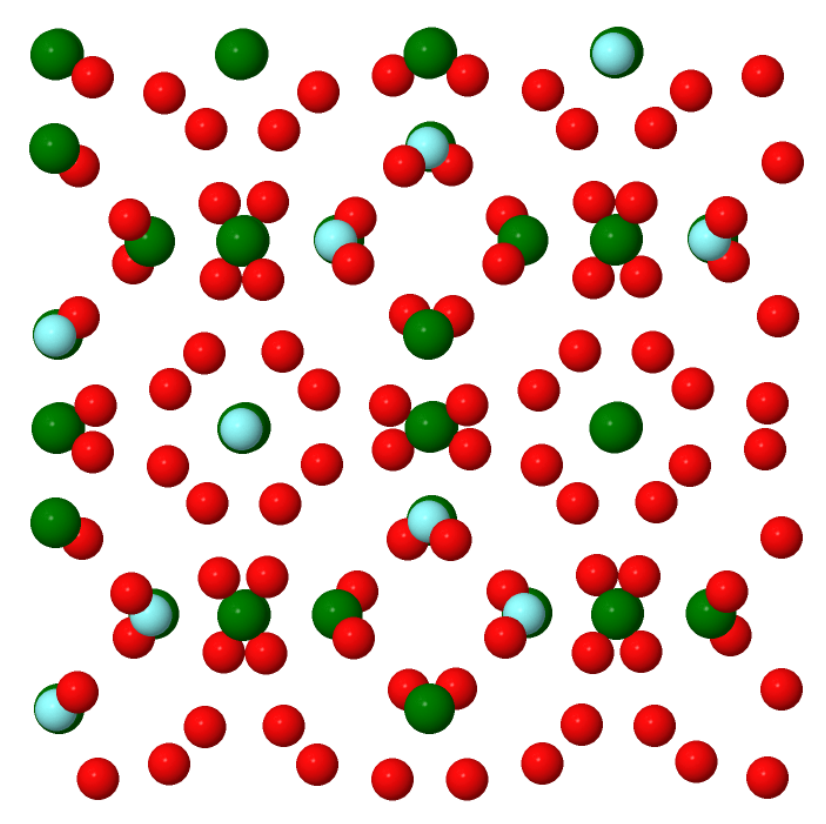

Figure 1 CONVENTIONAL UNIT CELL YAG SYMMETRY ON [ $\left.\begin{array}{lll}1 & 0 & 0\end{array}\right]$ FACE. Red:O, Green:Al, Blue:Y. (Image generated by Jmol [1])

Table 2 TWO-BODY PARAMETERS

\begin{tabular}{cccc}
\hline$i j$ & $A_{i j}(\mathrm{eV})$ & $B_{i j}^{-1}\left(\AA^{-1}\right)$ & $q_{i}(e)$ \\
\hline $\mathrm{O}-\mathrm{O}$ & 2449.44 & 3.44 & -2 \\
$\mathrm{Al}-\mathrm{O}$ & 1740.31 & 3.44 & +3 \\
$\mathrm{Y}-\mathrm{O}$ & 1250.85 & 2.86 & +3 \\
$\mathrm{Al}-\mathrm{Al}$ & 312.11 & 14.06 & \\
$\mathrm{Y}-\mathrm{Y}$ & 245.14 & 14.06 & \\
$\mathrm{Y}-\mathrm{Al}$ & 256.55 & 14.06 & \\
\hline
\end{tabular}




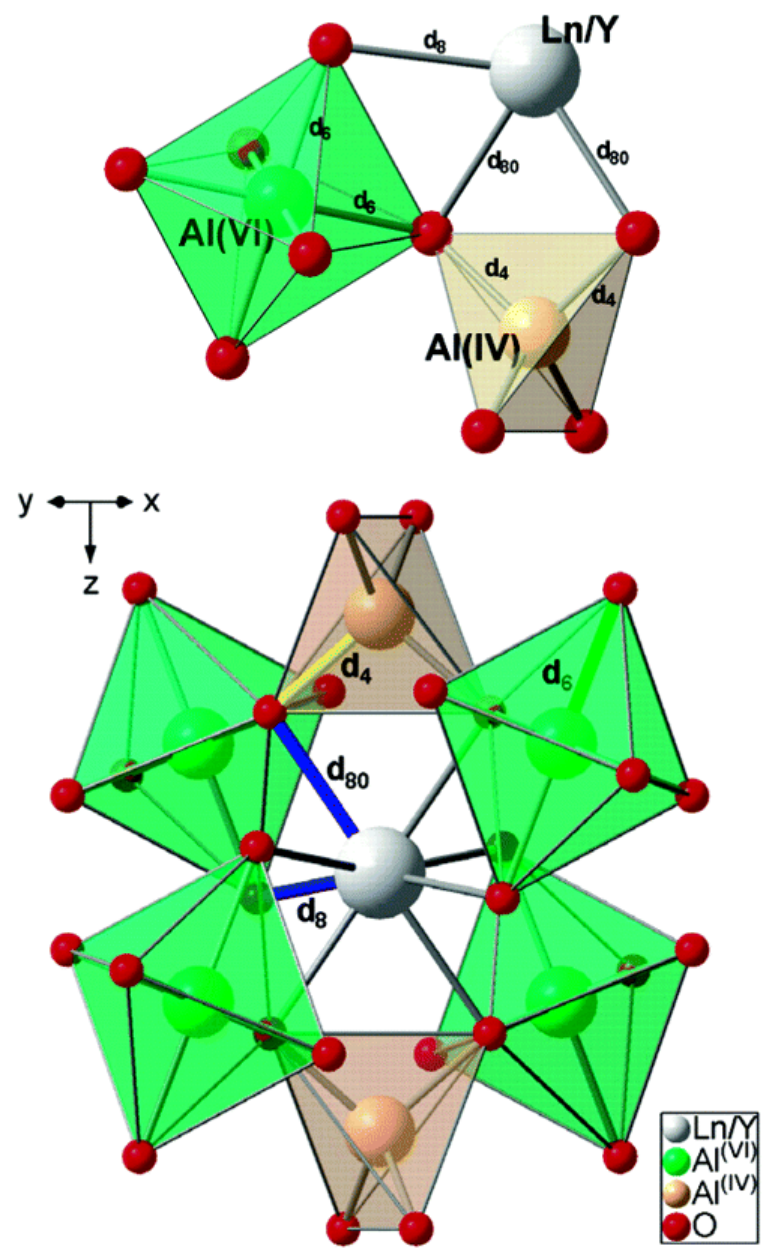

Figure 2 POLYHEDRA IN YAG [2] (with permission from the American Chemical Society) 
Table 3 THREE-BODY PARAMETERS

\begin{tabular}{ccccc}
\hline$j i k$ & $\lambda_{j i k}(\mathrm{eV})$ & $\gamma(\AA)$ & $r_{0, i j}(\AA)$ & $\theta_{0, j i k}\left({ }^{\circ}\right)$ \\
\hline $\mathrm{Al} / \mathrm{Y}-\mathrm{O}-\mathrm{Al} / \mathrm{Y}$ & 6.242 & 2.0 & 2.6 & 109.5 \\
$\mathrm{O}-\mathrm{Al}-\mathrm{O}$ & 149.324 & 2.8 & 3.0 & 109.5 \\
$\mathrm{O}-\mathrm{Y}-\mathrm{O}$ & 168.250 & 2.8 & 3.0 & 109.5 \\
\hline
\end{tabular}

In addition to pair potentials, three-body potentials were employed to enforce angular bonding between certain triplets of atoms as seen in Figure 2. For a triplet of atoms; $i, j$, and $k$; the three-body interaction, $U_{j i k}$, is expressed as

$$
U_{j i k}=\left\{\begin{array}{ll}
\lambda_{j i k} \exp \left(\frac{\gamma_{i j}}{r_{i j}-r_{i j, 0}}+\frac{\gamma_{i k}}{r_{i k}-r_{i k, 0}}\right) \Omega_{j i k} & r_{i j}<r_{i j, 0} \text { and } r_{i k}<r_{i k, 0} \\
0 & r_{i j} \geq r_{i j, 0} \text { or } r_{i k} \geq r_{i k, 0}
\end{array} .\right.
$$

$\Omega_{j i k}$ is an angular factor whose functional form depends on which triplet of atoms is being considered. For bonds where $j$ and $k$ represent oxygen atoms, and $i$ represents an aluminum or yttrium atom $(\mathrm{O}-\mathrm{Al} / \mathrm{Y}-\mathrm{O}), \Omega_{j i k}$ is expressed as

$$
\Omega_{j i k}=\left[\left(\cos \theta_{j i k}-\cos \theta_{j i k}^{0}\right) \sin \theta_{j i k} \cos \theta_{j i k}\right]^{2} .
$$

For triplets $\mathrm{Al} / \mathrm{Y}-\mathrm{O}-\mathrm{Al} / \mathrm{Y}, \Omega_{j i k}$ is

$$
\Omega_{j i k}=\left(\cos \theta_{j i k}-\cos \theta_{j i k}^{0}\right)^{2} .
$$

The parameters of Equations 2, 3, and 4 are in Table 3.

All simulations were performed using the June 8, 2011 version of the Large-scale Atomic/Molecular Massively Parallel Simulator (LAMMPS) package [16]. Furthermore, all simulations used the Verlet algorithm [17] for time integration. Periodic boundary conditions were imposed in all directions on all simulations to balance the repulsive forces 
from similarly charged two-body interactions. Periodic boundary conditions are also required to compute Coulombic interactions in reciprocal space.

The two-body interactions expressed by Equation 1 are implemented using the included Born-Mayer-Huggins potential [18] where Coulombic interactions greater than a specified cutoff were computed in k-space using a particle-particle particle-mesh (PPPM) solver. The LAMMPS tool, which does not include the proposed potential inherently, was modified to include the potential energies expressed by Equations 2, 3, and 4 (see Appendix).

\section{LAMMPS SOURCE MODIFICATION TEST}

The correctness of the source modification was tested by checking energy conservation in an NVE ensemble for one million timesteps (Figures II and II). A mistake in the code should not conserve energy at all, while an improper simulation parameter should result in poor energy conservation. The simulations ran with a $10^{-4}$ PPPM precision, a $4 \AA$ cutoff for non-Coulombic two-body interactions, and a $7 \AA$ cutoff for real space Coulombic interactions, and a $.002 \mathrm{ps}$ timestep. These parameters were found to conserve energy while maintaining decent execution speed. The simulation parameters used for the results presented here were the same or more stringent. 


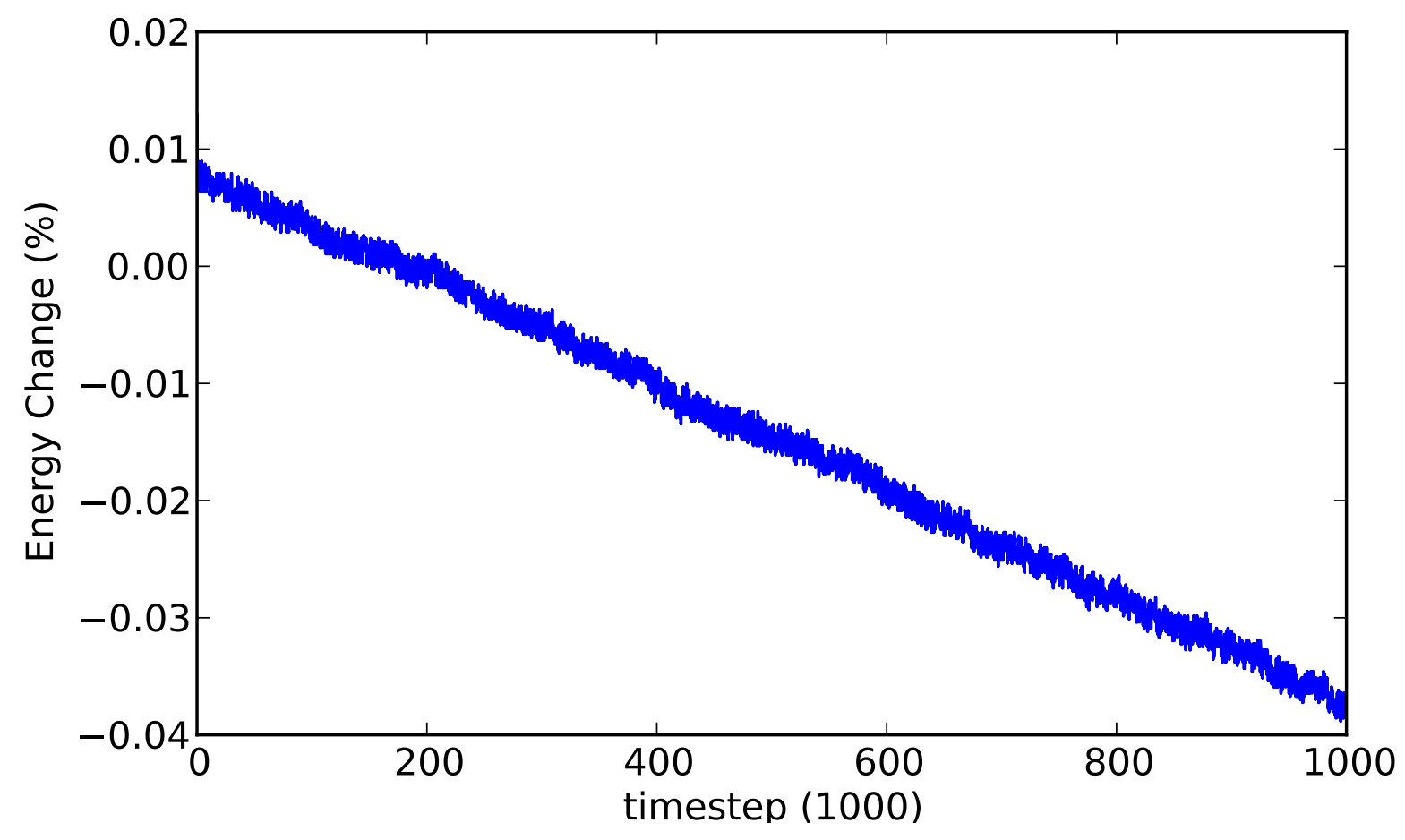

Figure 3 ENERGY CONSERVATION OF $2 \times 2 \times 2$ UNIT CELLS IN NVE ENSEMBLE FOR $x=0$ 


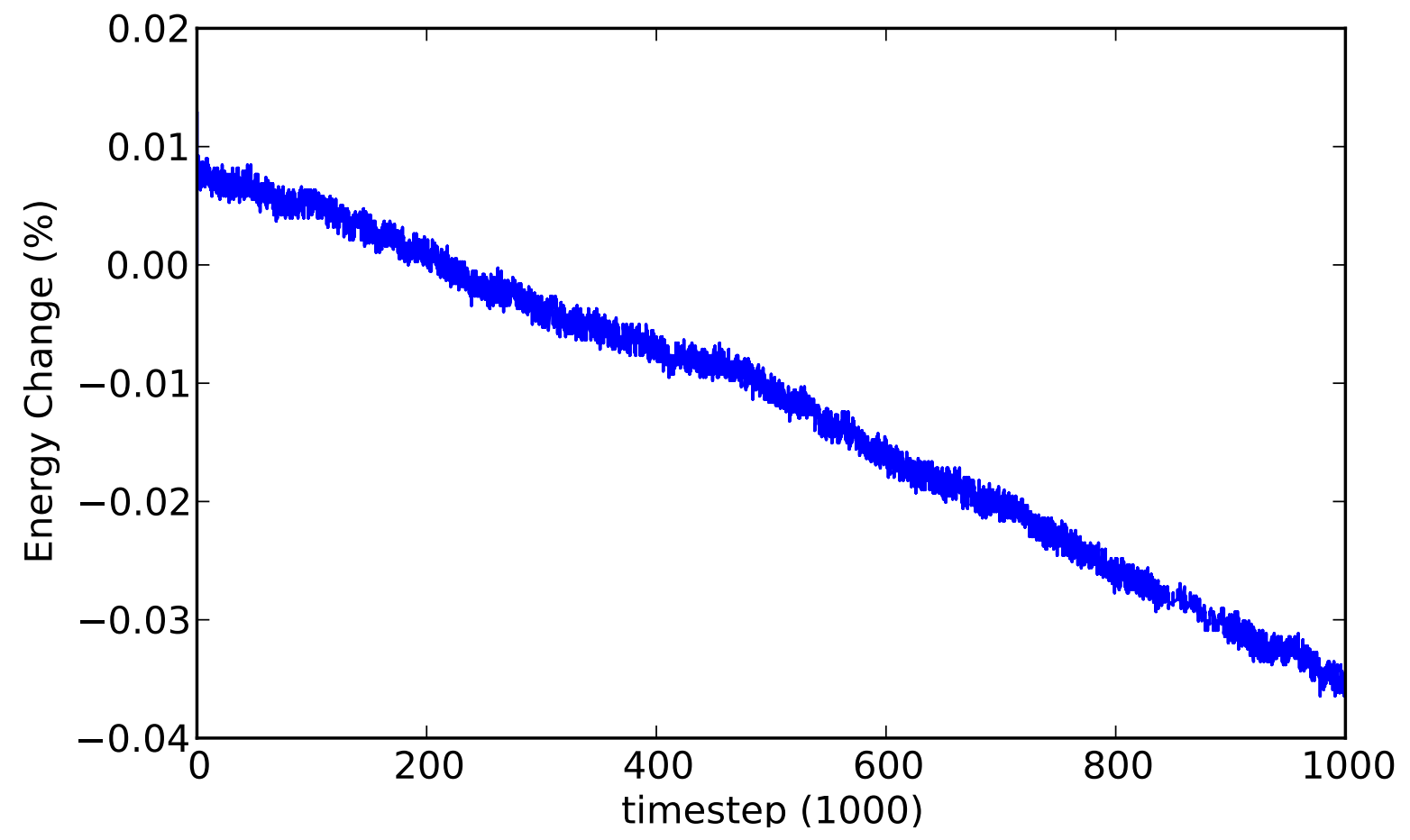

Figure 4 ENERGY CONSERVATION OF $2 \times 2 \times 2$ UNIT CELLS IN NVE ENSEMBLE FOR $x=50 \%$ 


\section{CHAPTER III}

\section{LATTICE CONSTANT, SPECIFIC HEAT CAPACITY, THERMAL EXPANSION}

This section describes how the constant-pressure specific heat, thermal expansion, and the lattice constant are found from simulated heating. The agreement of these quantities with experimental values indicates whether the interatomic potential is suitable for nontransport thermal properties.

\subsection{Simulation Setup}

$a, c_{p}$, and $\alpha$ are calculated from five simulations to represent a random aluminum substitution percentage of $0,25,50,75$, and 100 percent. The system size was $2 \times 2 \times 2$ unit cells (1280 atoms) for all simulations. Each simulation was initialized by selecting a normally distributed random velocity to give a temperature of $10 \mathrm{~K}$. Then, using NPT time integration, the system is thermostatted at $10 \mathrm{~K}$ and barostatted at 10 bars for 80,000 timesteps. Subsequently, the temperature is ramped up by $100 \mathrm{~K}$. After that, the system is again barostatted at 10 bars, thermostatted at the new temperature of $110 \mathrm{~K}$, and timeintegrated for another 80,000 timesteps. This process is repeated until the system reaches

a temperature of $3000 \mathrm{~K}$. Except for aluminum substitution, this simulation setup closely follows that of Jun [15] to allow for comparison.

The simulations ran with a 0.002 ps timestep, $10^{-4}$ PPPM precision, and a $7 \AA$ cutoff (for two-body interactions). The damping parameter on the thermostat was 1 ps while the damping parameter on the barostat was 10 ps. An additional drag factor of 1 was imposed to dampen unstable oscillations.

A time series for the total energy, temperature, pressure, and the lattice constant was collected from the simulation by a time averaging procedure: the quantities are sampled 


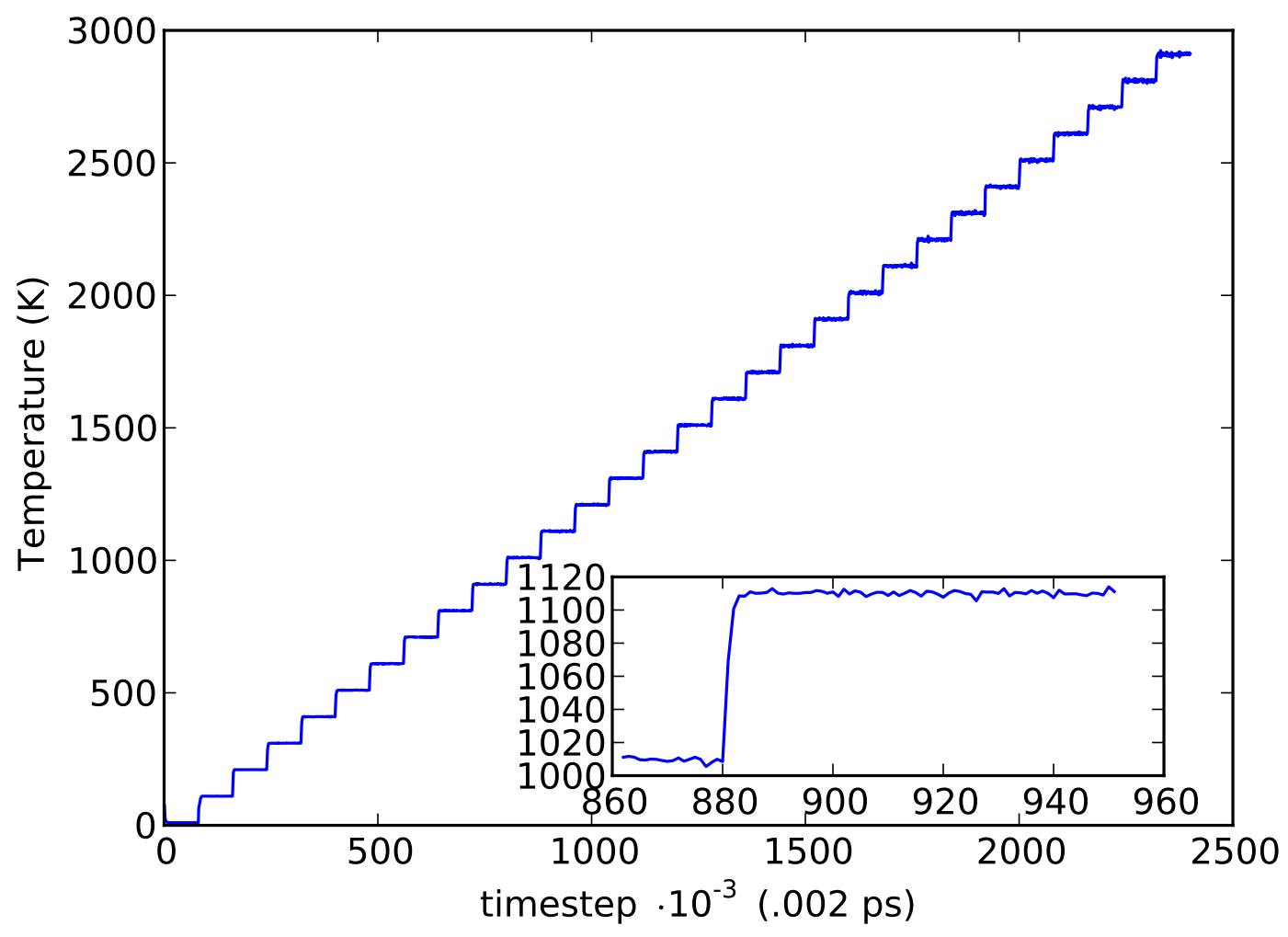

Figure 5 TEMPERATURE VS. TIME FOR 25\% Al SUBSTITUTION

every 10 timesteps 100 times, and subsequently averaged. This average is then recorded.

\subsection{Results and Discussion}

The stability of kinetic energy in the simulation can be seen in the time series in Figure 5 where the temperature is increased every 80,000 time steps. Variations in each temperature "step" were within $10 \mathrm{~K}$ as seen in the inset of Figure 5 while exhibiting almost no long-term transients over the 80,000 times steps. Therefore, we conclude that 80,000 time steps is sufficient to produce an equilibrated system for each temperature. Similarly, the lattice parameter, $a$, exhibited variations of $0.1 \%$ at most for each temperature.

From these data, the relationship between temperature and total energy can be es- 
timated, and it was found to be linear across the whole range of temperatures. Therefore, the specific heat, which is proportional to the slope of the energy/temperature relationship is constant in temperature.

$$
c_{p}=\frac{\Delta E}{m \Delta T}
$$

In a classical simulation, this finding is expected and the value should be similar to that of the law of Dulong and Petit $\left(c_{p}=3 N k_{B}\right.$, where $N$ is the number of oscillators). If we assume the density of YAG is $\rho=4.56 \mathrm{~g} / \mathrm{cm}^{3}$ and the lattice constant is $a=12.01 \AA$, then the Dulong and Petit specific heat reduces to

$$
c_{p}=3 \frac{160 \text { atoms/unit cell }}{12.01 \AA \text { Ànit cell }} \frac{k_{B}}{\rho}=0.84 \frac{\mathrm{J}}{\mathrm{g} \mathrm{K}}
$$

Figure 6 shows $c_{p}$ as a function of the percentage substitution. Note that the law of Dulong and Petit compares well with $0 \%$ substitution. However, the calculated $c_{p}$ of pure YAG is about $40 \%$ greater than the experimental value of $0.6 \mathrm{~J} / \mathrm{g} \mathrm{K}$. The discrepancy is probably due to imperfections in the measured materials. We do not have density information for the gallium substituted varieties, so direct comparison for the substituted varieties is not possible. Because $\Delta E / \Delta T$ were close to equal for all simulations, $c_{p}$ is primarily a function of mass as indicated by the trend for substituted systems. The molecular weight of YGG (100\% substitution) compared to YAG is 1.36 times larger. Therefore, the specific heat should be $1 / 1.36=0.74$ times smaller, or about $0.64 \mathrm{~J} / \mathrm{kg} \mathrm{K}$. This result is exactly the result of the molecular dynamics calculation.

A linear relationship was also found between temperature and lattice constant for all simulations. Therefore, the coefficient of thermal expansion $\alpha$, which is proportional to the slope of this relationship, is a constant.

$$
\alpha=\frac{\Delta V}{V_{0} \cdot 3 \Delta T} .
$$




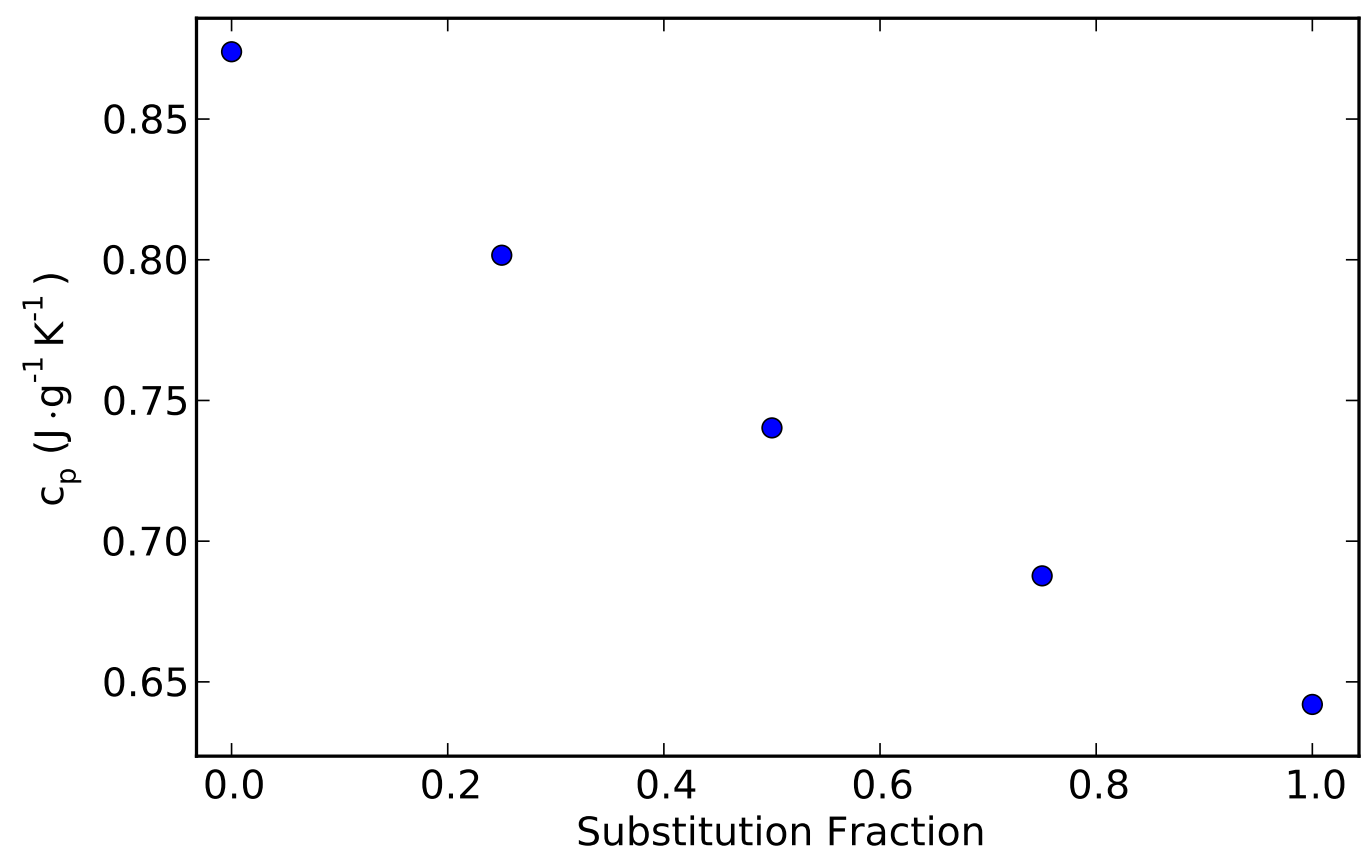

Figure $6 c_{p}$ VS. SUBSTITUTION FRACTION, $x$

The resulting thermal expansion of $\alpha=7.8 \cdot 10^{-6} \mathrm{~K}^{-1}$ for all simulations showed no dependence on aluminum substitution. This is expected given that the potential energy functions were the same for the substituted atoms. Nevertheless, this compares well with the established figure of $7.0 \times 10^{-6} \mathrm{~K}^{-1}$ for pure YAG. As a result, a fit of the lattice constant data as a function of temperature yields

$$
a=9.67 \cdot 10^{-5} T\left(\frac{\AA}{\mathrm{K}}\right)+12.4(\AA) .
$$

So at $300 \mathrm{~K}$, the lattice constant is $12.43 \AA$ which is $3.6 \%$ greater than published figures [14] but only $0.6 \%$ greater than the result from MD simulations of Reference [15]. However, we can not validate the linear thermal expansion coefficient for gallium gallium-substituted YAG, and these values should be questioned. 


\section{CHAPTER IV}

\section{MELTING TEMPERATURE}

The melting temperature can inferred from three independent measures: 1) system potential energy, 2) the radial distribution function (RDF), and 3) the Lindemann index $\delta$ [19]. All of these metrics indicate that atoms have changed their local environment and all are a function of temperature.

The RDF is defined as

$$
g(r)=\lim _{d r \rightarrow 0} \frac{p(r)}{4 \pi\left(N_{\text {pairs }} / V\right) r^{2} d r}
$$

where $r$ is the distance between a pair of particles, $p(r)$ is the average number of atom pairs found at a distance between $r$ and $r+d r, V$ is the volume of the system, and $N_{\text {pairs }}$ is the number of unique pairs of atoms. The RDF can be interpreted as the probability of finding an atom at a distance $r$ relative to that of an ideal gas. In the solid phase, RDFs are characterized by peaks which become less pronounced with increasing temperature [20] indicating less structural order. Therefore, as the substance melts, the distance between atomic sites becomes blurred.

The system-averaged Lindemann index is an average over unique atom pairs as expressed in Equation 10.

$$
\delta=\frac{2}{N(N-1)} \sum_{i, j(i \neq j)}^{N} \frac{\sqrt{\left\langle\mathbf{r}_{i j}^{2}\right\rangle-\left\langle\mathbf{r}_{i j}\right\rangle^{2}}}{\left\langle\mathbf{r}_{i j}\right\rangle}
$$

It measures disorder and the mobility of the atoms relative the distances between them. During a heating process, the index increases linearly with temperature. Deviation from 
linearity is taken as a change in the structure.

Finally, changes in the trend of potential energy as a function of temperature also indicates structural changes.

\section{Simulation Setup}

The simulations devised to predict melting temperature are based on the simulations that produced data for lattice constant, specific heat and thermal expansion. However, because periodic boundaries are used to mimic an infinite crystal, there are no surfaces to initiate melting. As described previously, this lack of defect structure delays the onset of melting to physically unrealistic values. Therefore, vacancies were introduced by removing 6 random aluminum atoms in a $2 \times 2 \times 2$ unit cell system. To study the effects of number fo defects on the melting process, another simulation of the same size was conducted with 15 randomly selected aluminum atoms removed. In both simulations, the heating process started from $1500 \mathrm{~K}$ and proceeded to $3500 \mathrm{~K}$ in $10 \mathrm{~K}$ increments. Each temperature was kept for 100,000 time steps.

The potential energy, temperature, pressure, and the lattice constant were collected in time from the simulation by the same averaging procedure as before. The time step, potential cutoff, PPPM precision, and thermostatting were also the same. Meanwhile, the RDF was calculated every 10 timesteps. To calculate the Lindemann index, the coordinates for 20 randomly chosen atoms were sampled every 5 timesteps. In a separate simulation it was found that the summands in the Lindemann index follow a well-defined distribution so that the Lindemann index evaluated with only 20 atoms was within $2 \%$ of the evaluation using all atoms. Sampling the atom trajectories in this way saves significant computational resources. 


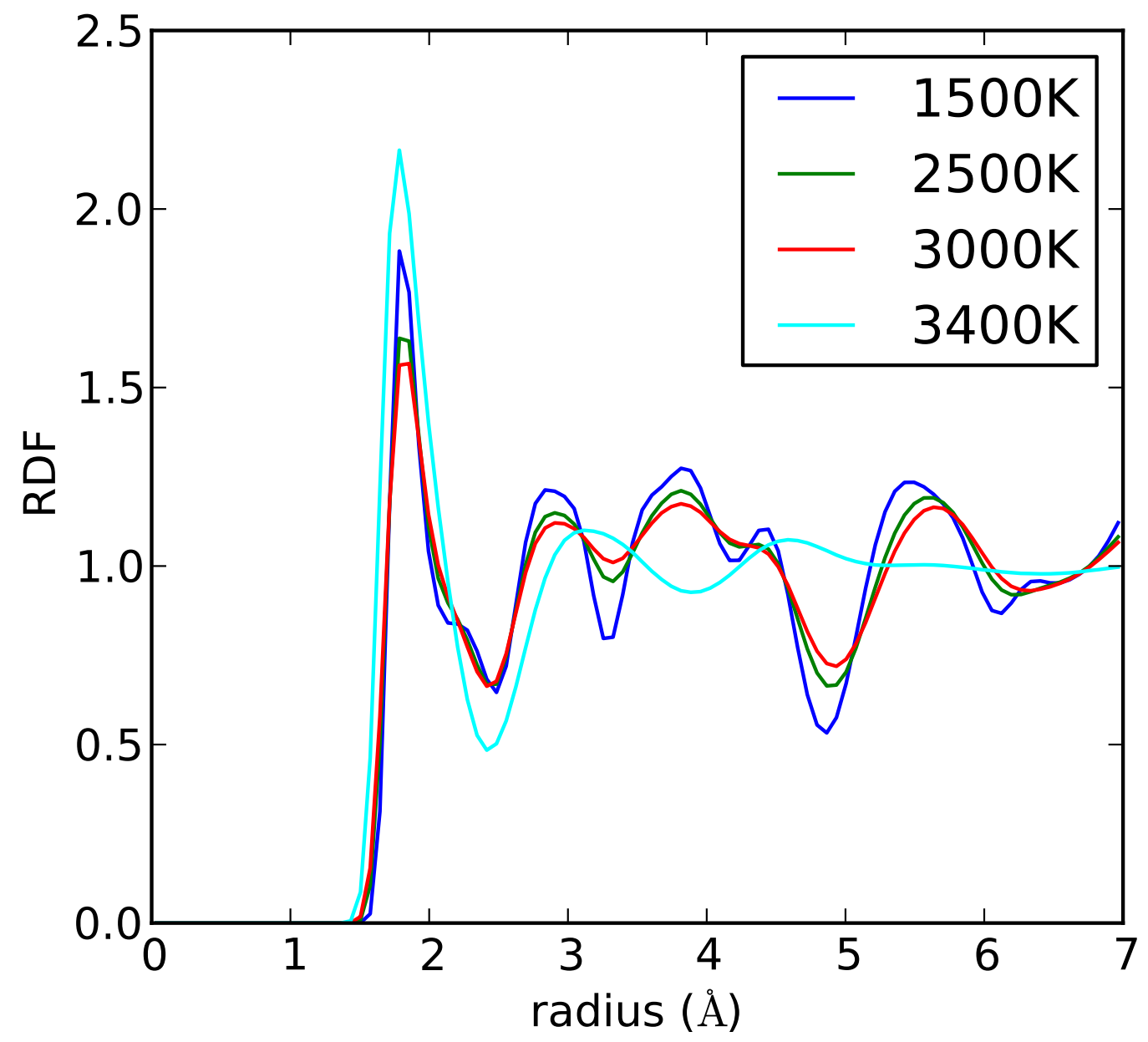

Figure 7 RADIAL DISTRIBUTION FUNCTION FOR 6 EVACUATED ALUMINUM ATOMS

\subsection{Results and Discussion}

The RDF of both simulations were similar at the temperatures plotted in Figure 7. We see a systematic but small loss of order between $1500 \mathrm{~K}$ and $3000 \mathrm{~K}$. However, the difference in the RDF between $300 \mathrm{~K}$ and $3400 \mathrm{~K}$ is dramatic indicating that atoms are staring to migrate within the lattice and that the melting temperature is between these values. This transition indicates a loss of order beyond $4 \AA$.

The potential energy also shows a dramatic change between the temperatures iden- 


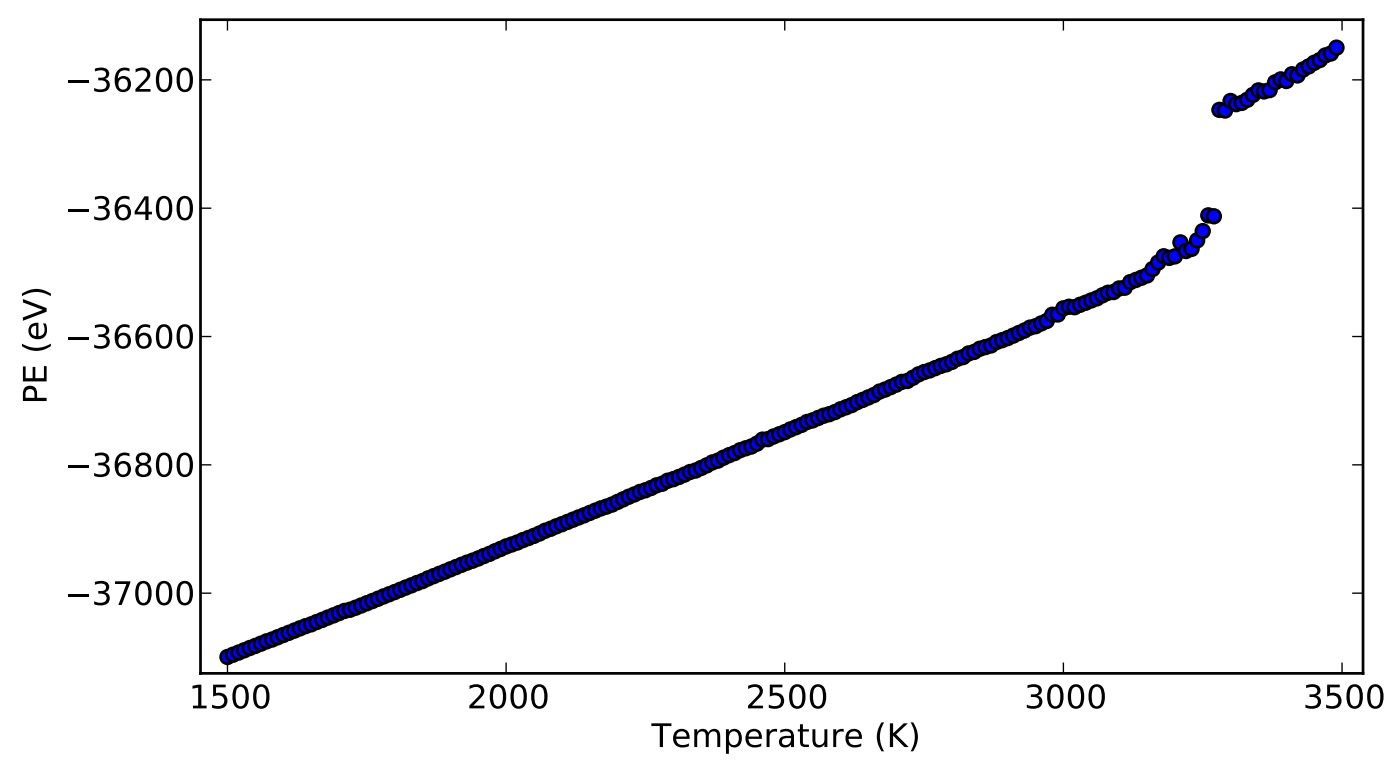

Figure 8 POTENTIAL ENERGY FOR 6 EVACUATED ALUMINUM ATOMS

tified by the RDF. An abrupt transition is seen by looking at a plot of potential energy versus temperature [21] as in Figures 8 and 9. Once the atoms begin to migrate relative to each other, which is the onset of melting, the potential energy remains large as the atoms are not constrained to the bottom of the potential well formed between atoms. The transitions occurred at two temperatures: one at about $3075 \mathrm{~K}$ and the other at approximately $3250 \mathrm{~K}$. As expected the more highly defected material melts sooner than the less defected material. These results corroborate those found from the RDF analysis.

Meanwhile, the Lindemann index for the systems, shown in Figures 10 and 11, shows transitions occurring at temperatures below $3000 \mathrm{~K}$ by deviating from linearity. However the transitions were hundreds of degrees above the experimental melting temperature of YAG $(2213 \mathrm{~K})$. Nonetheless, the deviation from linearity is due to the coexistence of the liquid and solid state in the material (dynamic coexistence) [21] where the YAG ceases to be a crystal and is instead broken down into mobile clusters. These clusters must have all elements $(\mathrm{Y}, \mathrm{Al}$, and $\mathrm{O})$ in order to be cohesive since the force between like atoms 


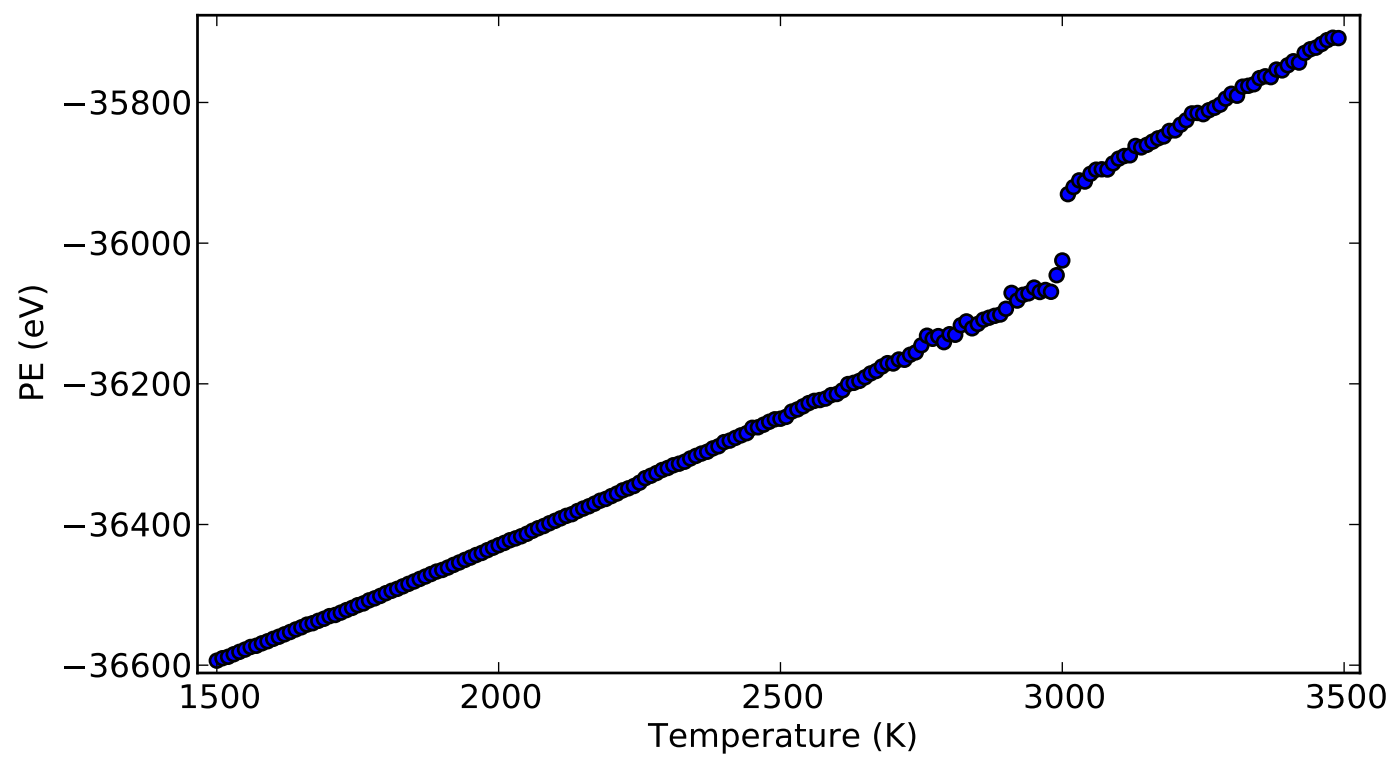

Figure 9 POTENTIAL ENERGY FOR 15 EVACUATED ALUMINUM ATOMS

is repulsive. So, oxygen may be seen as a binder of yttrium and aluminum. This explains why the RDF does not show much change through most of the temperature range. The $\mathrm{RDF}$ captures the relatively short-range order of these clusters while they may be mobile in the simulation. The defectiveness of the structure is more apparent with the more defective system as seen Figure 11 in comparison to Figure 10. The system with more vacancies is less stable. So, precisely determining the melting temperature from these simulations is difficult given this transition region over a wide temperature range. Furthermore, the configurations may be stable at a certain temperature in the timescale of the simulation but not in the timescale required for melting [7]. Even further, once the YAG structure is broken, the potentials used are no longer strictly applicable. 


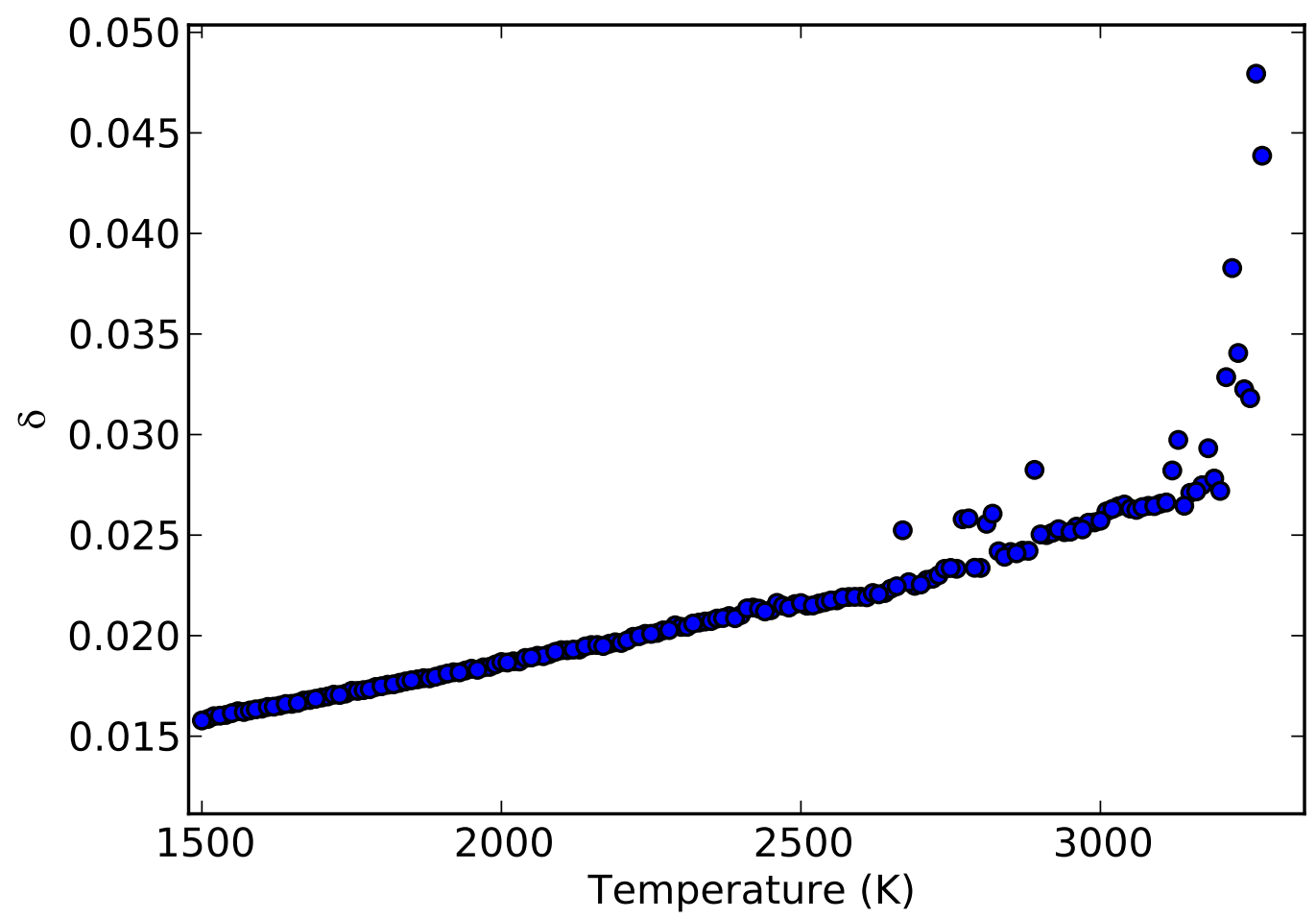

Figure 10 LINDEMANN INDEX FOR 6 EVACUATED ALUMINUM ATOMS

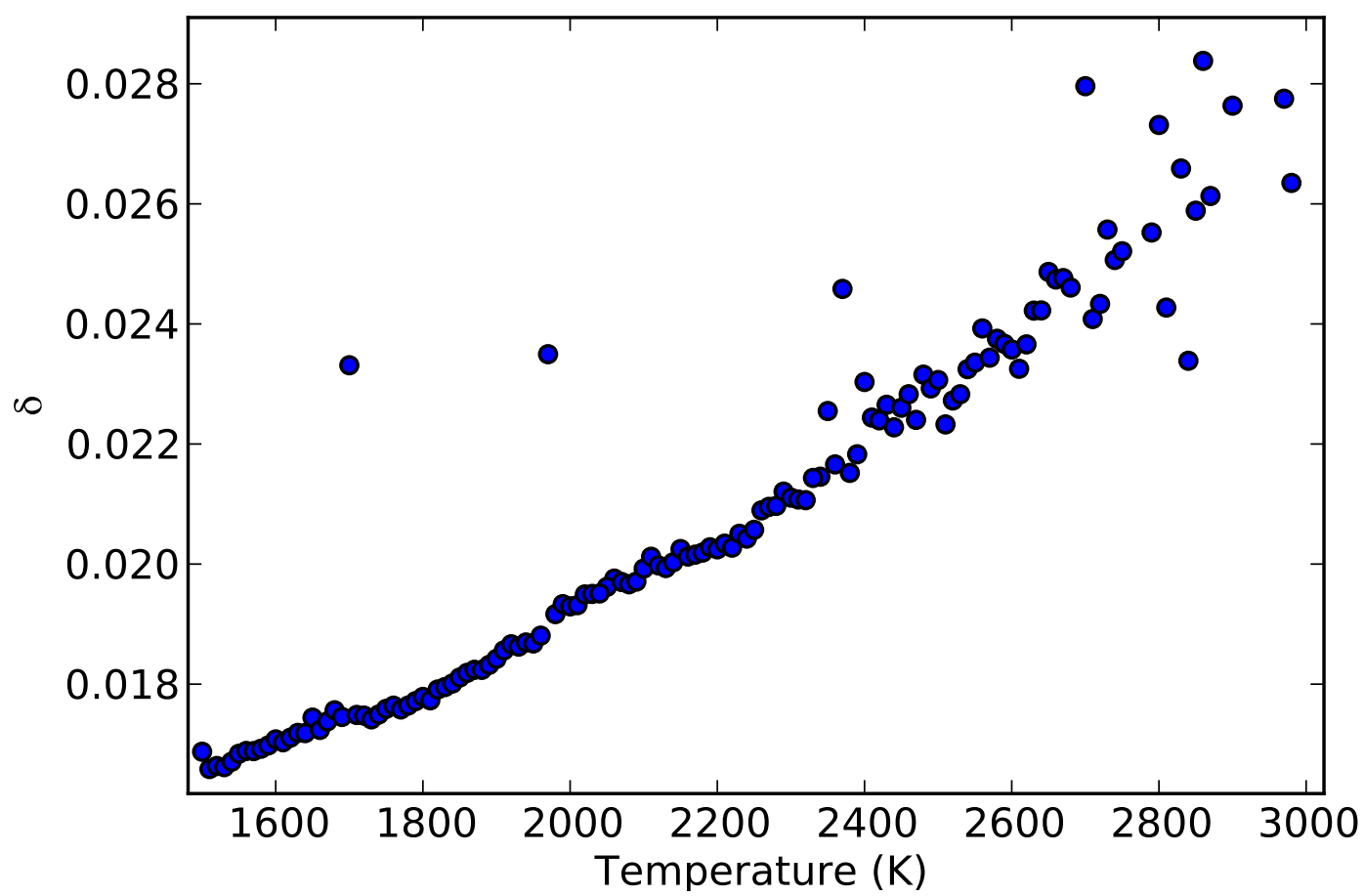

Figure 11 LINDEMANN INDEX FOR 15 EVACUATED ALUMINUM ATOMS 


\section{CHAPTER V}

\section{THERMAL CONDUCTIVITY FROM NON-EQUILIBRIUM SIMULATIONS}

The simulations described in this section measure thermal conductivity in the [100] direction. Since YAG is arranged in a cubic structure, its thermal conductivity is given by a diagonal tensor

$$
\mathbf{k}_{i j}=\left|\begin{array}{ccc}
k & 0 & 0 \\
0 & k & 0 \\
0 & 0 & k
\end{array}\right| .
$$

Therefore, the thermal conductivity tensor is fully described by this one measure.

An effective thermal conductivity, $k$, is obtained by imposing a temperature gradient so that Fourier's law of conduction in one dimension may be evaluated directly. In one set of simulations the temperature gradient is specified directly. In another set of simulations, the temperature gradient is specified indirectly by specifiying a temperature difference between each side of a slab. In either case, the thermal conductivity is calculated as

$$
k=-\frac{\dot{q} x}{A_{c}} \frac{d x}{d T}
$$

where $\dot{q}$ is the amount of energy added to the hot reservoir or removed from the cold reservoir to maintain the temperature. The derivative is the inverse of the temperature gradient that is a result of the simulation. 


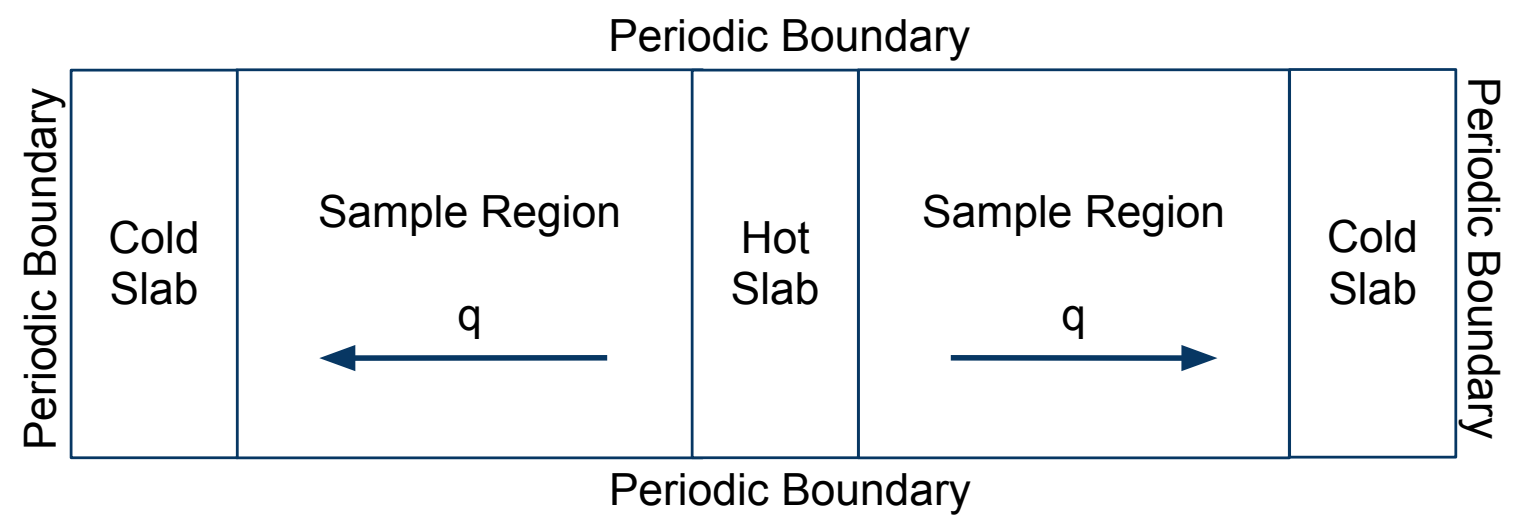

Figure 12 NON-EQUILIBRIUM MD CONFIGURATION

\subsection{Simulation Setup}

Two temperature gradients were imposed in a symmetric configuration, as represented in Figure 12, since the simulations feature periodic boundary conditions. This configuration ensures that the hot slab is not exposed to the cold slab at the simulation boundary.

For all simulations, the lattice spacing was $12.43 \AA$. Furthermore, the hot slab was $8 \times 1 \times 1(\mathrm{~L} \times \mathrm{H} \times \mathrm{D})$ unit cells, while each cold slab was $4 \times 1 \times 1$ unit cells. As such, the atoms in each $4 \times 1 \times 1$ region were assigned to four thermostats. The thermostats were applied to the hot slab and cold slab such that the average temperature of the temperature gradient was fixed at $300 \mathrm{~K}$.

Simulations were varied in the size of the sample region, in random aluminum substitution fraction, $x$, and in the temperature difference between the hot and cold themostats, $\Delta T$, for one set of simulations. The sample sizes were $4,6,8,12,16,24$, and $32 ; x$ was 0 , 50, and 100 percent; and $\Delta T$ was 40,100 , and $150 \mathrm{~K}$ for a total of 63 simulations. In the other set of simulations, instead of specifiying $\Delta T$, the temperature gradients were $0.4,0.6$, 0.8 , and $1 \mathrm{~K}$ per lattice constant. The other variables were the same giving 84 simulations.

An initial linear temperature gradient was imposed over the sample region with a random gaussian velocity distribution. The temperature gradient is then maintained by the 


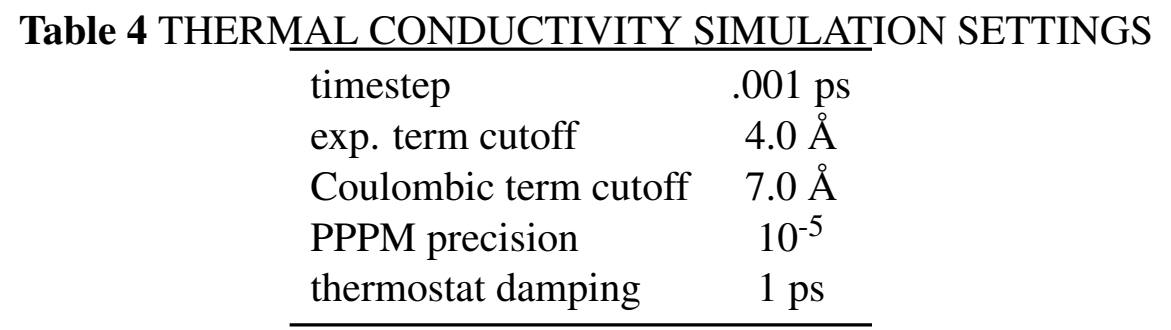

thermostats for $10^{6}$ timsteps $(10 \mathrm{~ns})$. The region controlled by the thermostats are timeintegrated with the NVT integrator, while the sample region was time-integrated with the NVE integrator.

During the run, the average temperature in each unit cell is calculated every 10 timesteps. The time average is then recorded for 100 of these calculations. Meanwhile, the data from the thermostats are recorded every 1000 timesteps. The data from the thermostats represent the cumulative energy change of the system due to their action. In particular, here it represents an energy addition or subtraction due to the flow of energy from the hot slab to the cold slab.

Table 4 lists the simulation settings. The cutoffs apply to the two-body potential (Equation 1).

\subsection{Results and Discussion}

A linear temperature profile is exhibited in all simulations when averaged over their run time including systems of 4 unit cells wide as in Figure 13.

To calculate thermal conductivity according to Fourier's law (Equation 12), the slope from a linear fit to the sample region from each side of the temperature profile is averaged to obtain $\Delta x /\left(A_{c} \Delta T\right)$. In addition, $\dot{q}_{x}=\Delta E / \Delta t$ where $\Delta E$ is represented by the energy difference of $E_{t s}^{a v g}$ between the start and end of the simulation in steady state and $A_{c}$ is the sample cross-sectional area. 


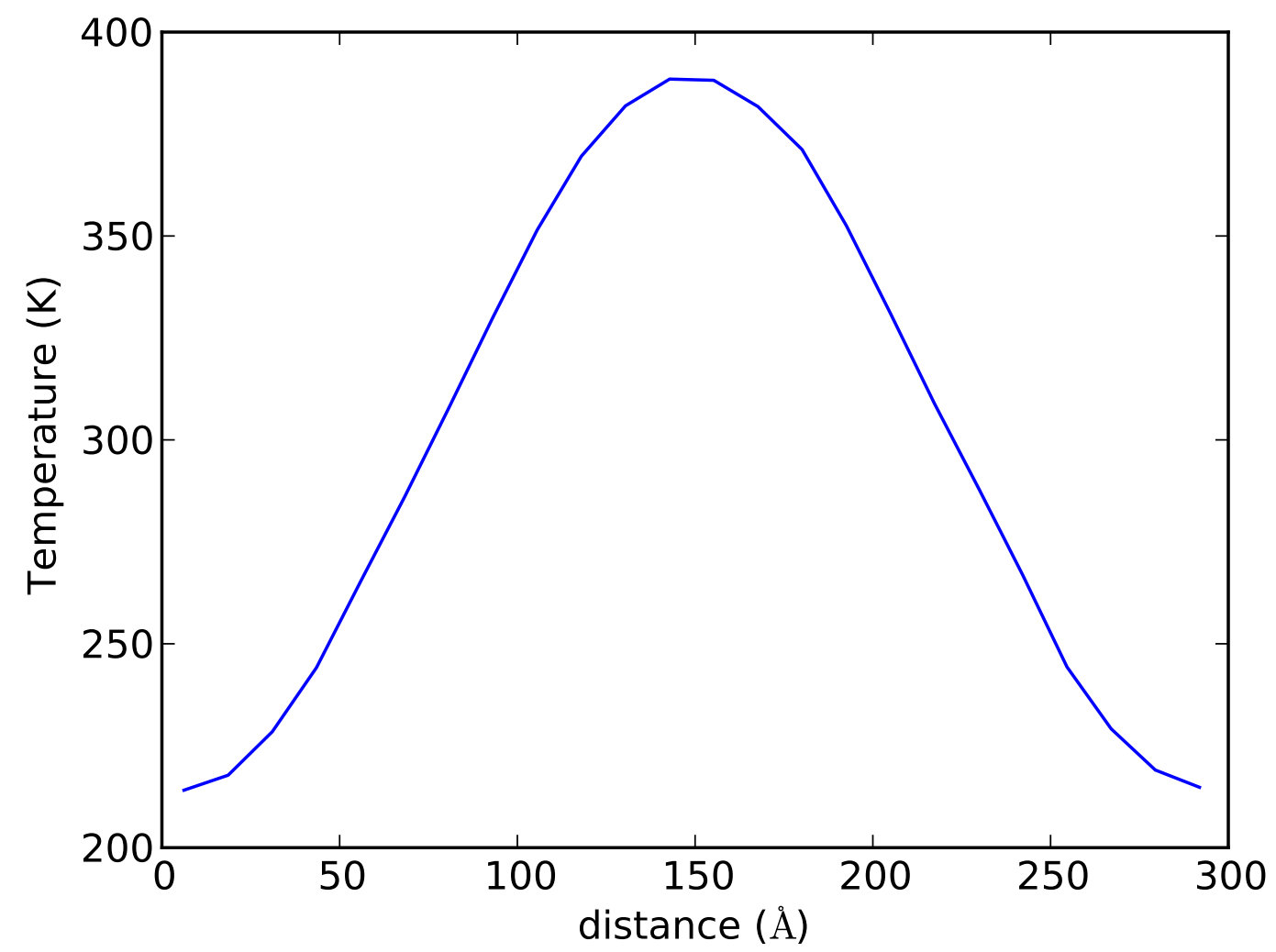

Figure 13 AVERAGE TEMPERATURE PROFILE FOR A SAMPLE SIZE 4 UNIT CELLS WIDE, WITH $50 \%$ Al SUBSTITUTION, $\Delta T=150 \mathrm{~K}$ 
Figure 14 shows the results of the thermal conductivity calculation for the set of simulations specifying $\Delta T$ while Figure 15 shows the calculations for the set of simulations specifiying temperature gradient. The error bars represent the standard deviation in calculating the thermal conductivity for different temperature gradients or temperature differences. For pure YAG, the trend is approaching (the lower end of) its accepted bulk value of $10 \mathrm{~W} \cdot \mathrm{m}^{-1} \mathrm{~K}^{-1}[12]$. All trends of a certain substitution fraction (varying system size) can be explained by simplified kinetic theory: the relationship between thermal conductivity, specific heat capacity, group velocity, and mean free path is given by

$$
k=\frac{1}{3} v \Lambda c_{v}
$$

Because system size is the only variable, the decrease in thermal conductivity with decreasing system size is due to either decreased mean free path or a reduction in available vibrational modes for heat transfer. Long wavelength modes are more efficient at transferring energy, so in systems that involve only 4 unit cells, only 4 acoustic modes (vibrations among unit cells) are possible. Transport, therefore, relies primarily on optical modes (vibrations within unit cells), which are known to be inefficient thermal carriers. Moreover, the acoustic modes will be of short wavelength, whose group velocity will be small. In a system whose size is comparable to the mean free path, reduced thermal conductivity is observed due to the boundary scattering, which reduces the effective mean free path. However, we do not know what the intrinsic mean free path of YAG is; therefore, we can not make strong statements about this effect. Nevertheless, phonon mean free paths are often larger than a few unit cells. So it seems plausible that this effect is present as well.

Meanwhile, group velocity is needed to fully explain the trends for a certain system size (varying substitution fraction). Assuming acoustic phonons are the primary thermal carriers, the lower thermal conductivity of YAG fully substituted with gallium compared to pure YAG can be explained by the difference in their phonon dispersions: as gallium is a 
heavier element than aluminum, the group velocity of fully substituted YAG will be lower, resulting in lower thermal conductivity. Still, decreased mean free path explains why the thermal conductivity for $x=50 \%$ is less than $x=0 \%$ and $x=100 \%$. The presence of gallium atoms distributed randomly in the structure represents mass-difference impurity phonon scattering centers that reduce the mean free path of phonons.

The increase in thermal conductivity with increasing system length is expected due to the contribution of longer wavelength phonon modes. However, the trend does not converge over the simulated lengths, and simulating larger lengths is computationally impractical. While an inverse $\mathrm{k}$ versus inverse length is a typical approach to estimate the infinite size or bulk thermal conductivity from a reduced system size, others have noted similar difficulties in getting the data to appear linear or converge [22]. These artifacts seems to appear in relatively complex systems such as buckyballs, and YAG certainly qualifies as a complex system. The lack of convergence is probably due to new modes that are introduced as the system size increases rather than simply adding more frequencies of the same modes, which is what happens in smaller, simpler systems. In addition, the mean free path of various modes are likely different. Therefore, as the system size is increased, a host of scattering parameters come into play making the dominant mechanism become non-linear. Nevertheless, the data show a definite trend that appears to converge to expected bulk values for thermal conductivity of YAG. 


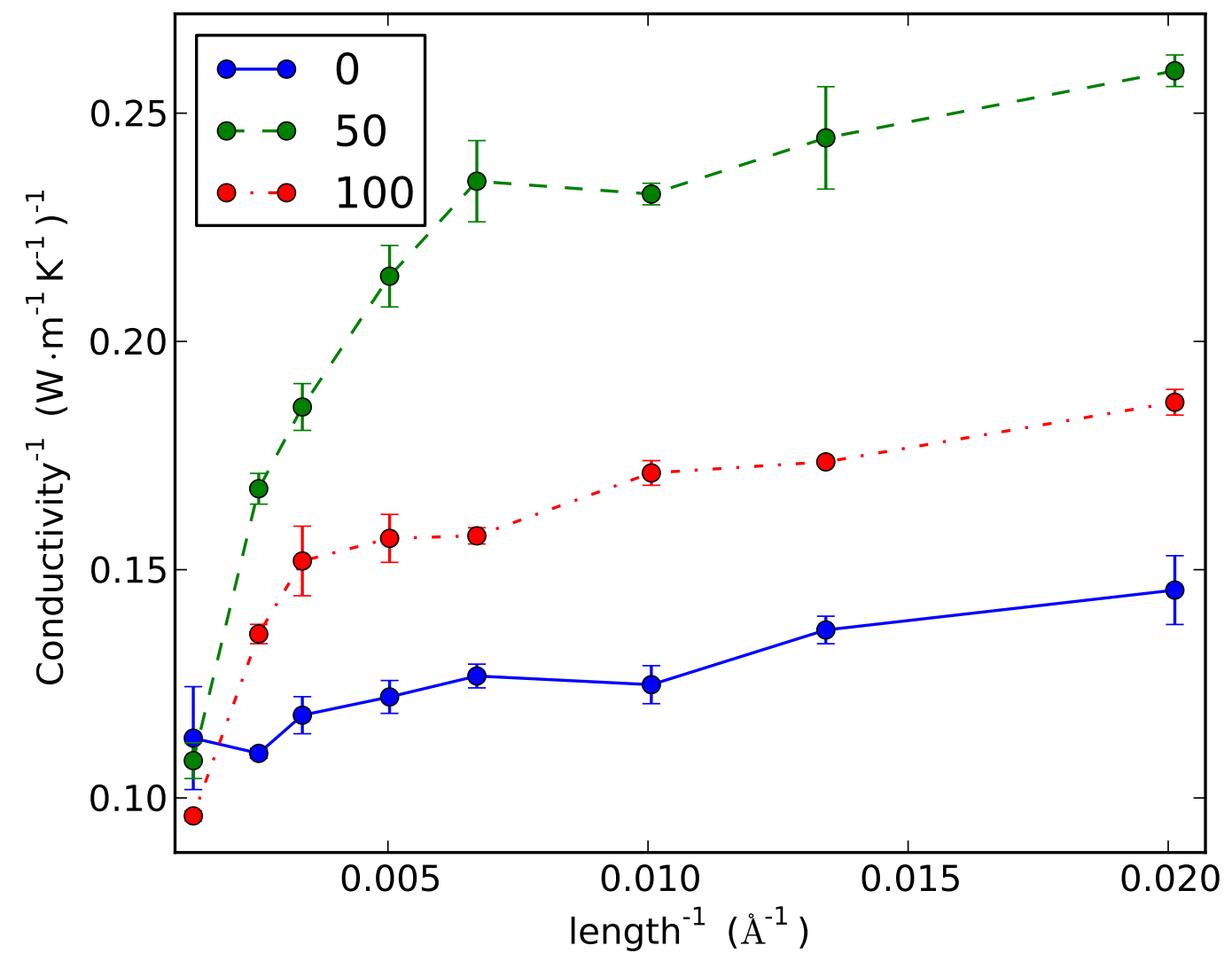

Figure 14 NON-EQUILIBRIUM THERMAL CONDUCTIVITY TRENDS SPECIFYING TEMPERATURE DIFFERENCE FOR $x=0,50$, AND $100 \%$. 


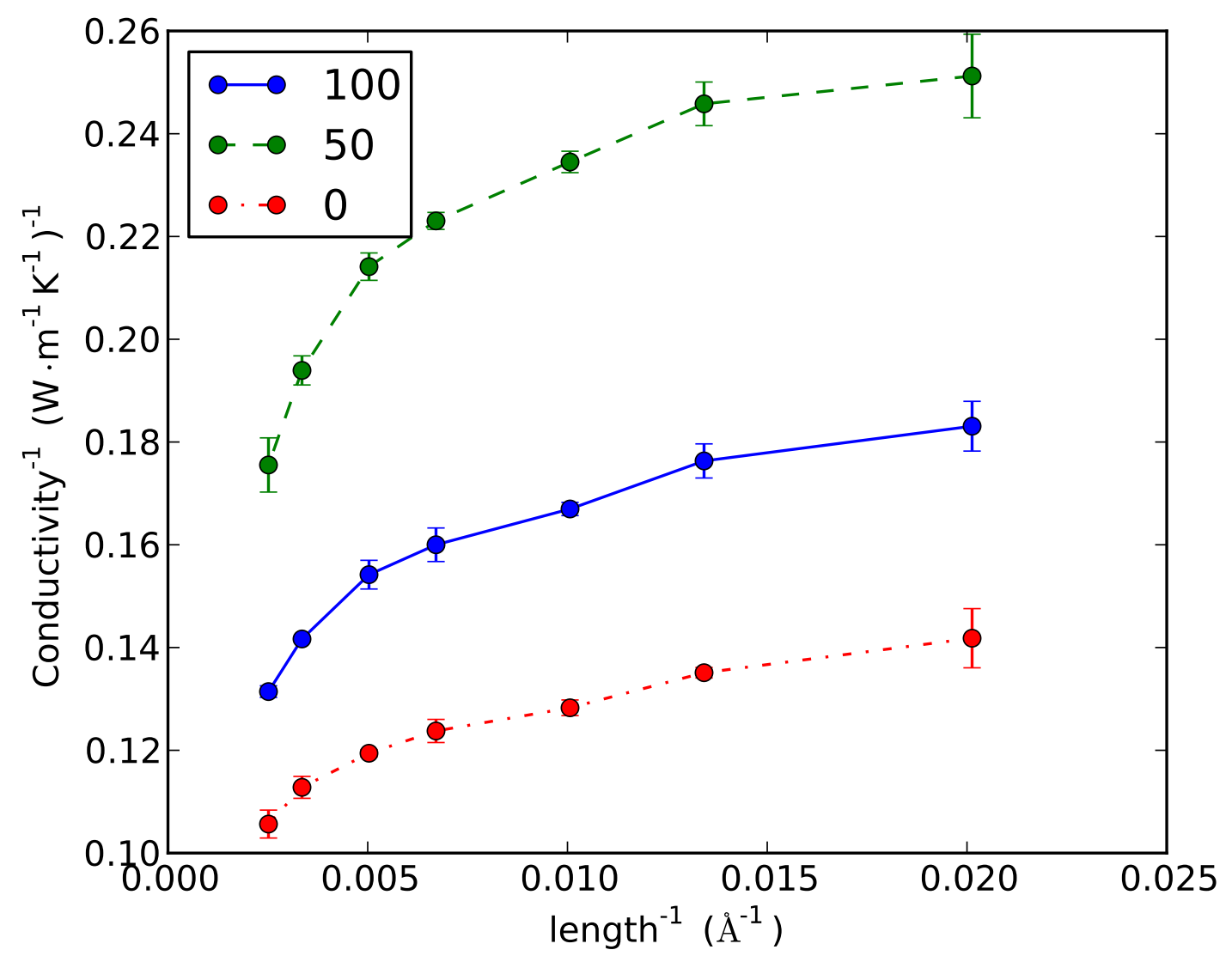

Figure 15 NON-EQUILIBRIUM THERMAL CONDUCTIVITY TRENDS SPECIFYING SPECIFYING TEMPERATURE GRADIENT FOR $x=0,50$, AND $100 \%$. 


\section{CHAPTER VI}

\section{CONCLUSIONS}

The results for pure YAG shows good agreement with experimental values for constant-pressure specific heat, thermal expansion, and non-equilibrium thermal conductivity at $300 \mathrm{~K}$, suggesting that the interatomic potentials that were originally developed for mechanical properties are nearly suitable or thermal properties. Of course the quality of the potential must be graded against the quality of the thermal property that needs to be estimated. For order of magnitude and trends, we are confident that these potentials are suitable.

The melting of YAG is not a well-defined process occuring over a wide temperature range. Modeling the surface of YAG, where interatomic potentials are not readily available, is needed to better simulate its melting process. In this case, new potentials are likely needed, so defects can be treated more rigorously. Consideration of this relationship is important in high temperature-transient applications.

The relationship between thermal properties and and aluminum substitution may not be robust. Although qualitative trends were established and corroborated, the simulations do not consider slight modifications to the interatomic potentials due to changes in bonding between gallium and oxygen versus aluminum and oxygen. These changes in bonding could be important, although the foregoing analysis was unable to discern whether

the interatomic potentials were sufficient. Therefore, experimental thermal properties for gallium-substituted YAG are needed to confirm the YAG model used in this work. 


\section{APPENDIX}

\section{LAMMPS SOURCE MODIFICATION}

In order to compute three-body energies and their forces for this work, code for two potentials was added based on the included Stillinger-Weber potentials (composed of two and three-body interactions). One implemented the three-body interaction formed by Equation 2 and Equation 3 while zeroing the original two-body interaction. The other involved only zeroing the two-body part of the Stillinger-Weber potentials since the threebody interaction formed by Equation 2 and Equation 4 is compatible with the original code. The following code shows the implementation of Equations 2 and 3 ('mbmh' potential in LAMMPS script).

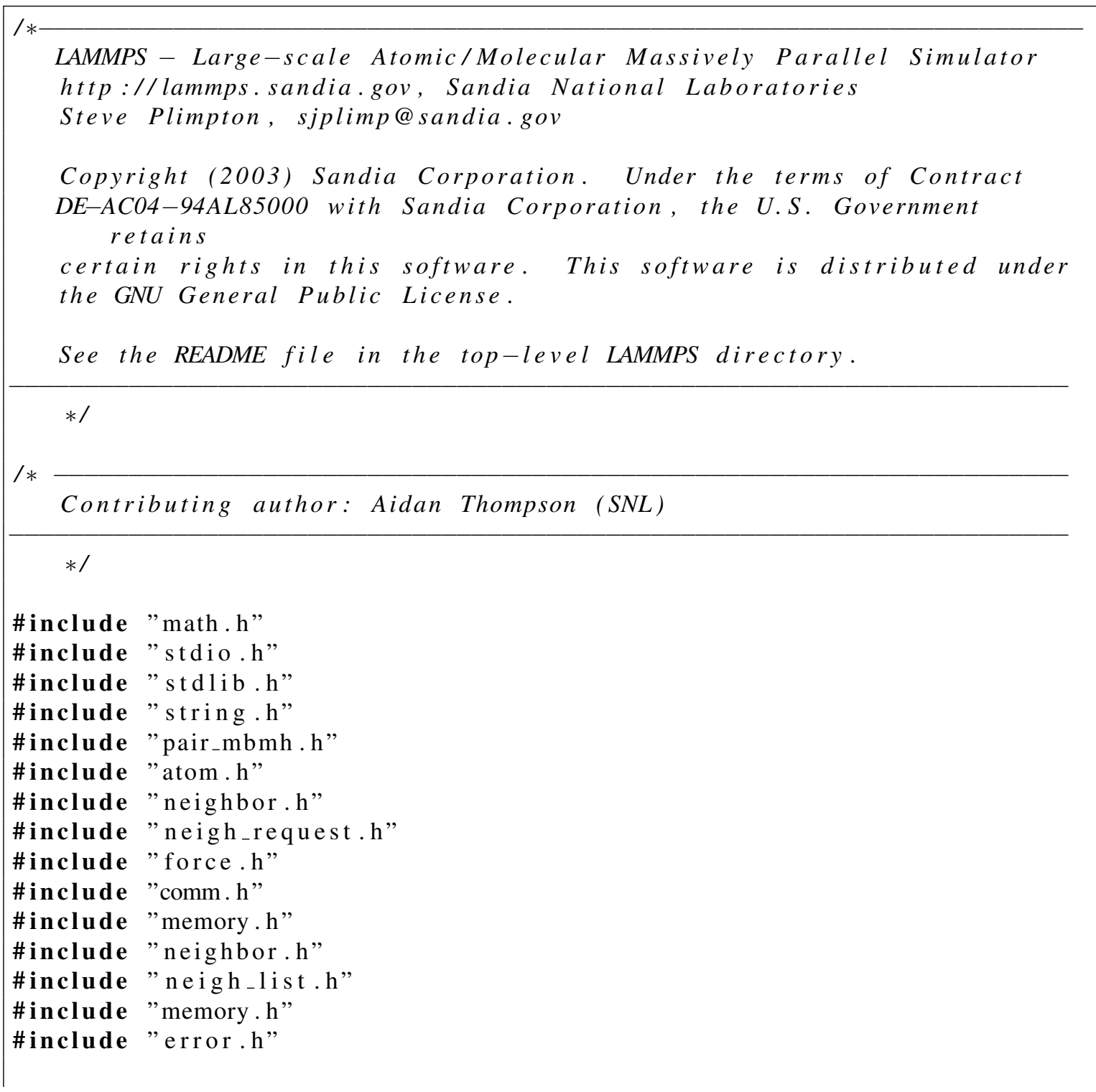




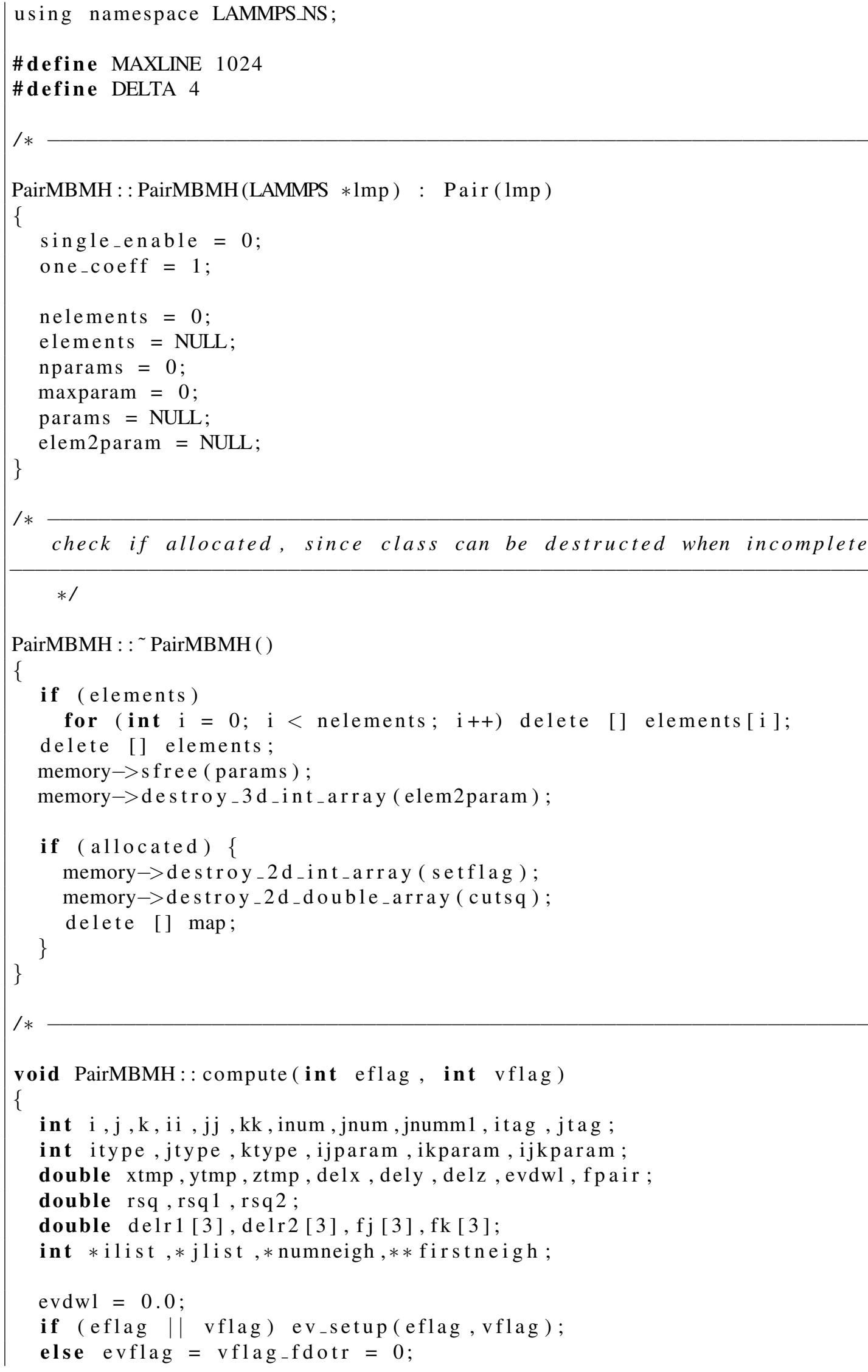




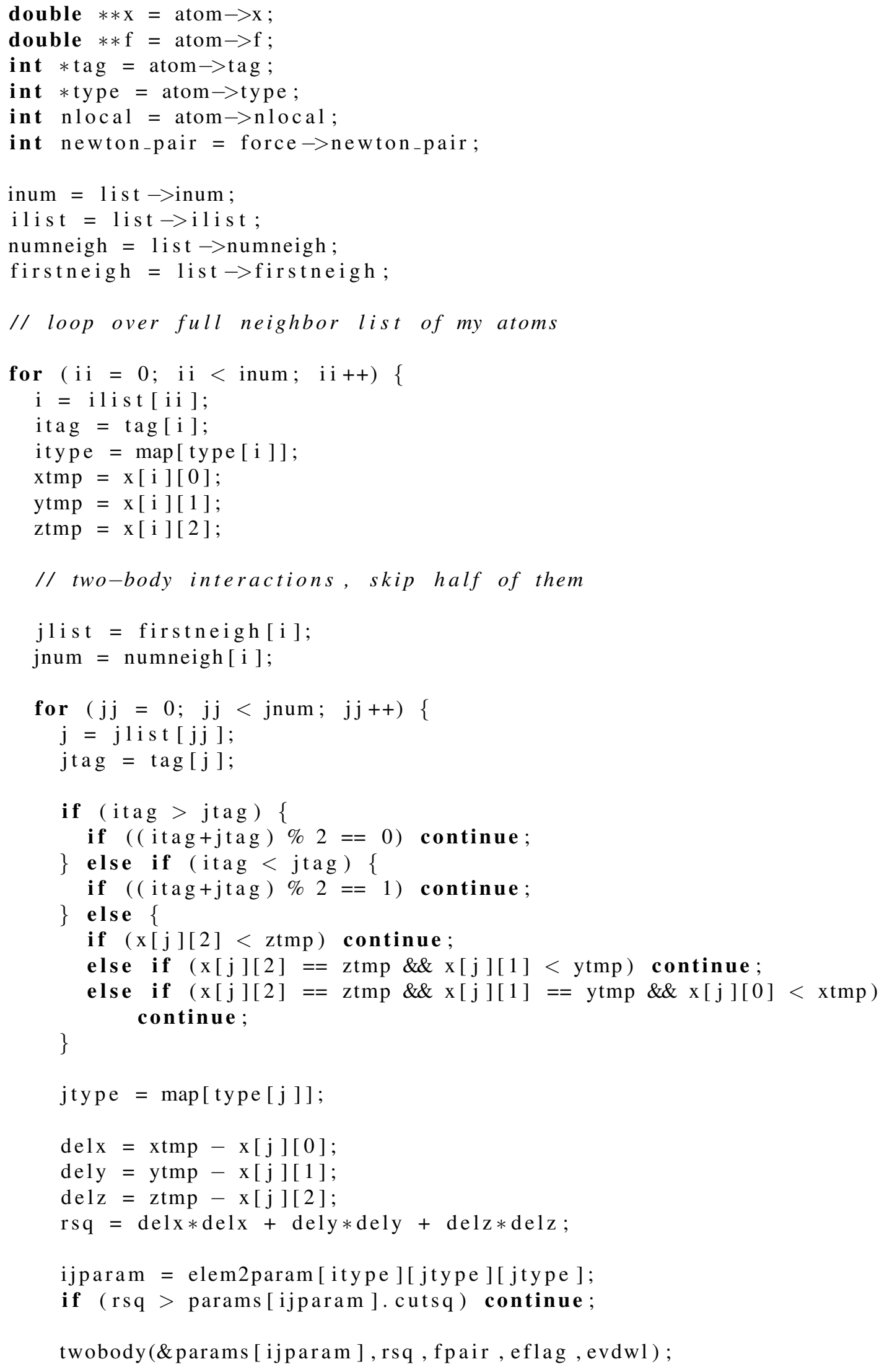




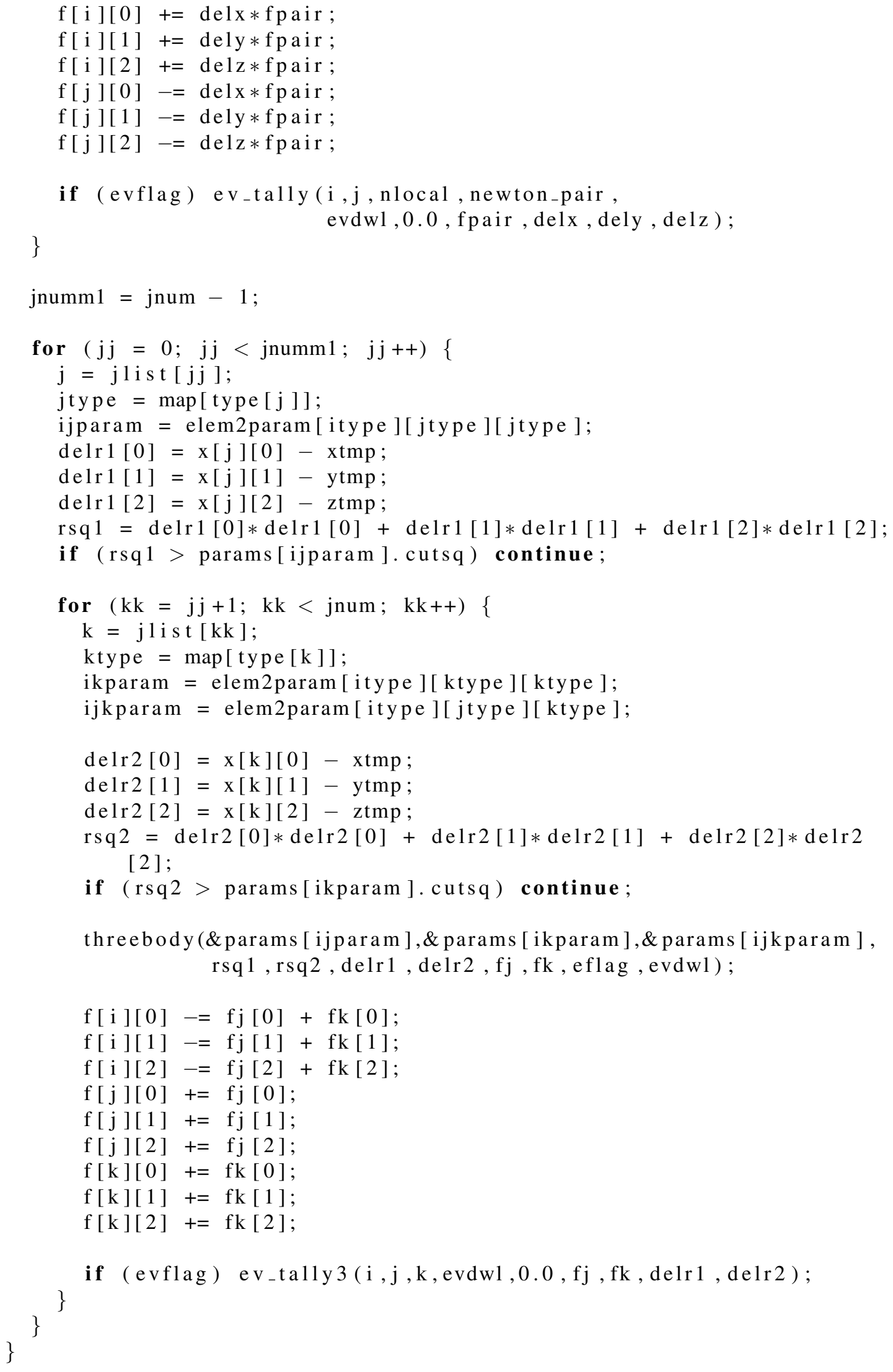




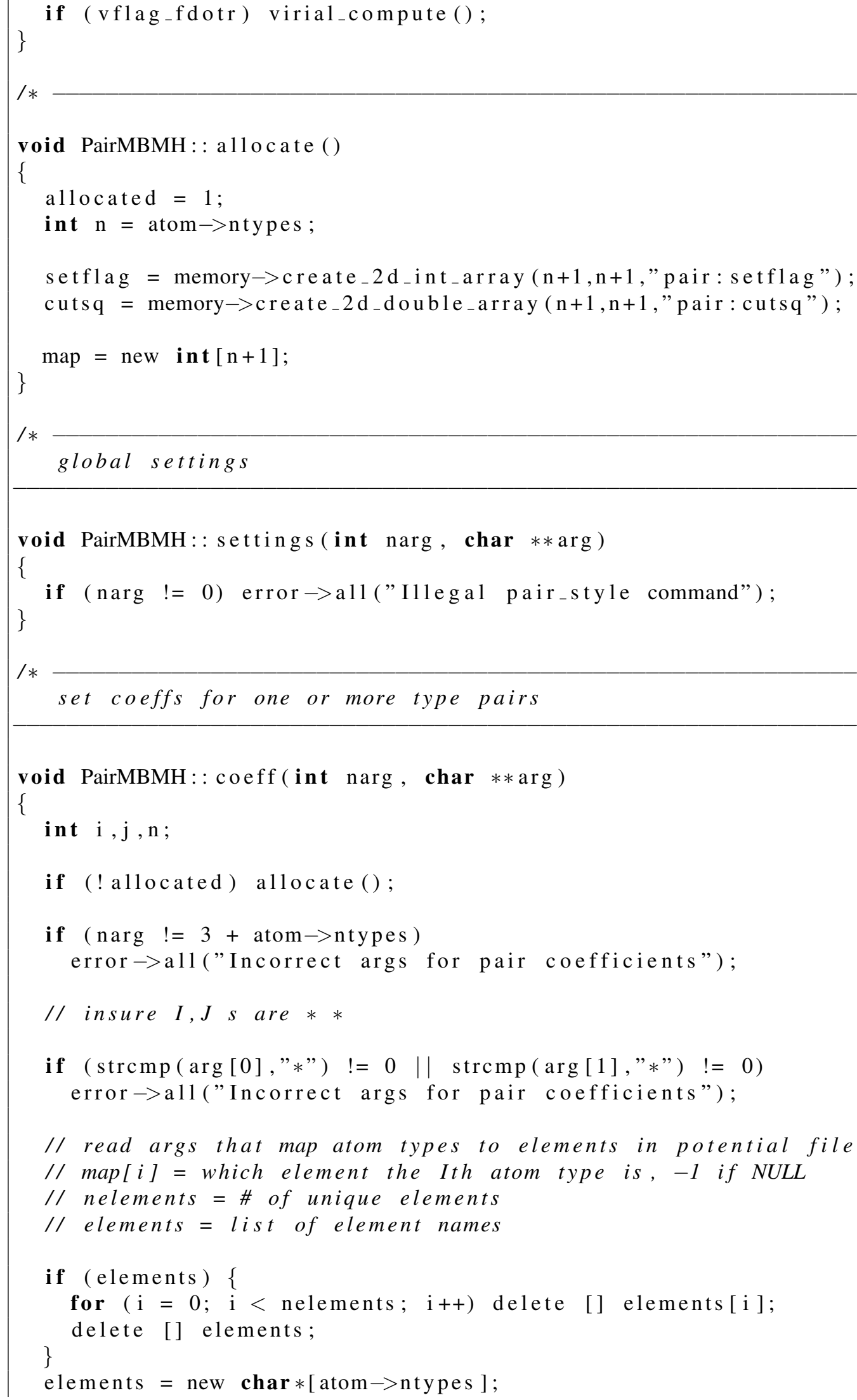




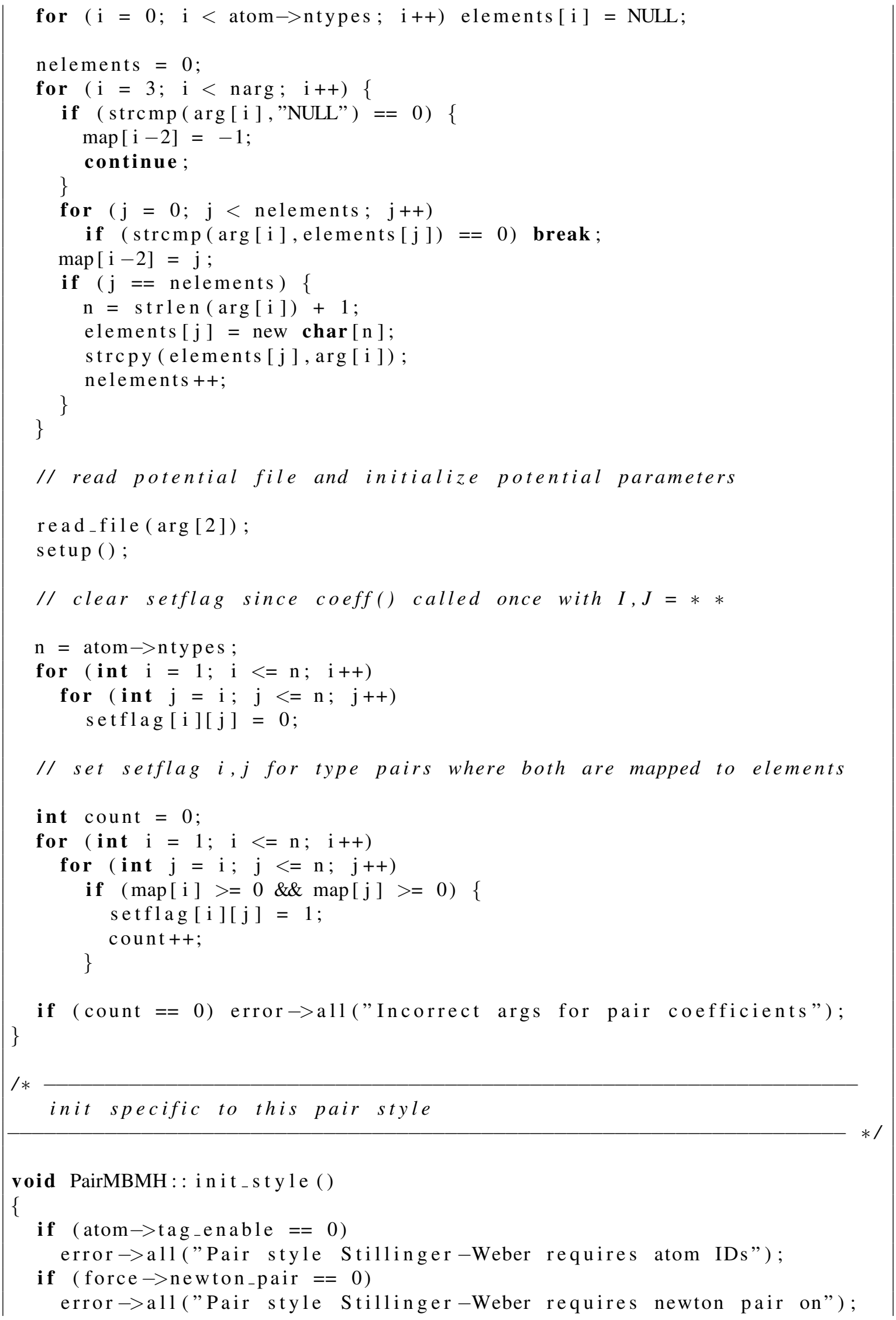




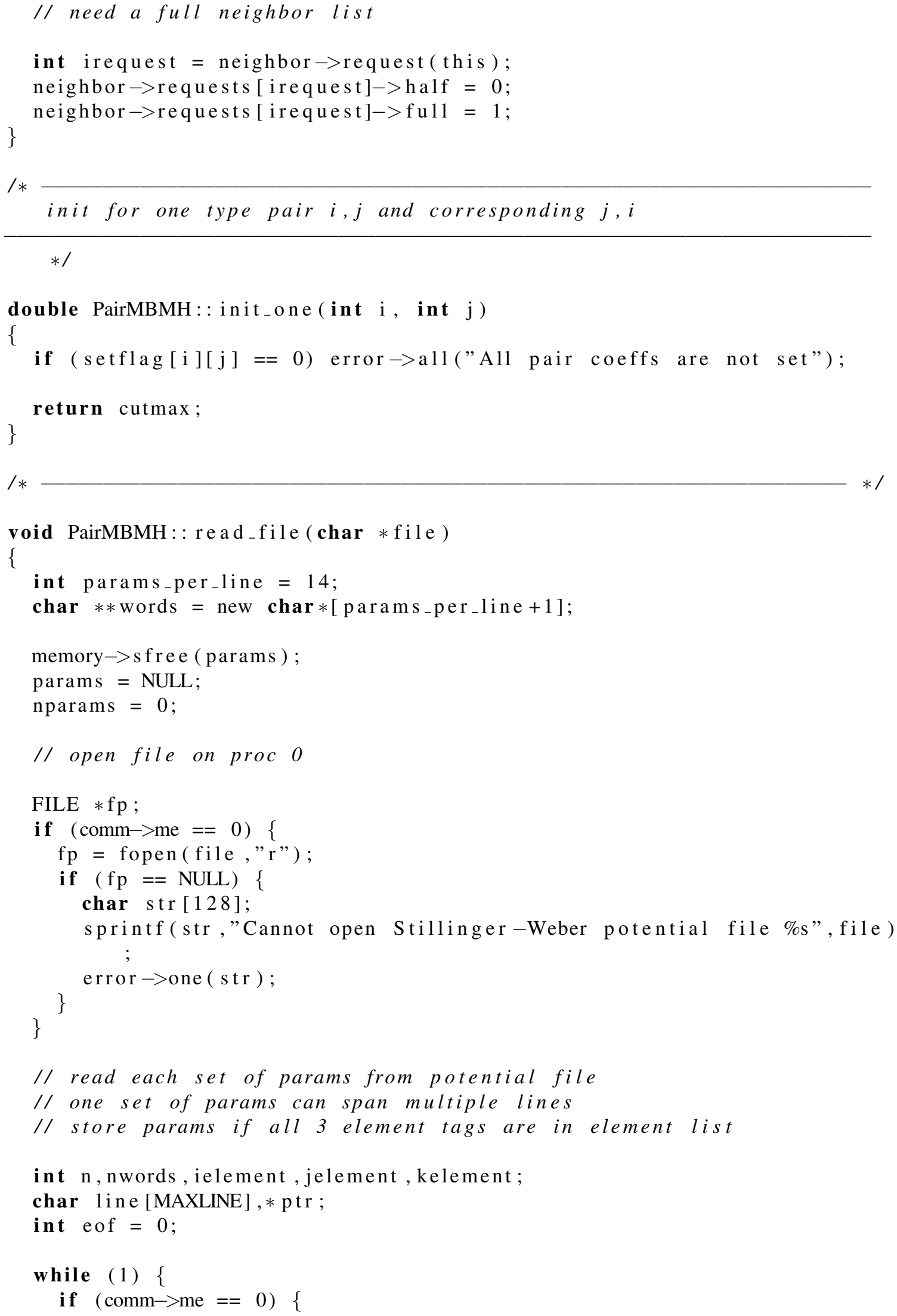

int n, nwords, ielement, jelement, kelement;

char line [MAXLINE], $*$ ptr;

int e of $=0$;

while (1) \{

if $($ comm $-\rightarrow$ me $==0)\{$ 


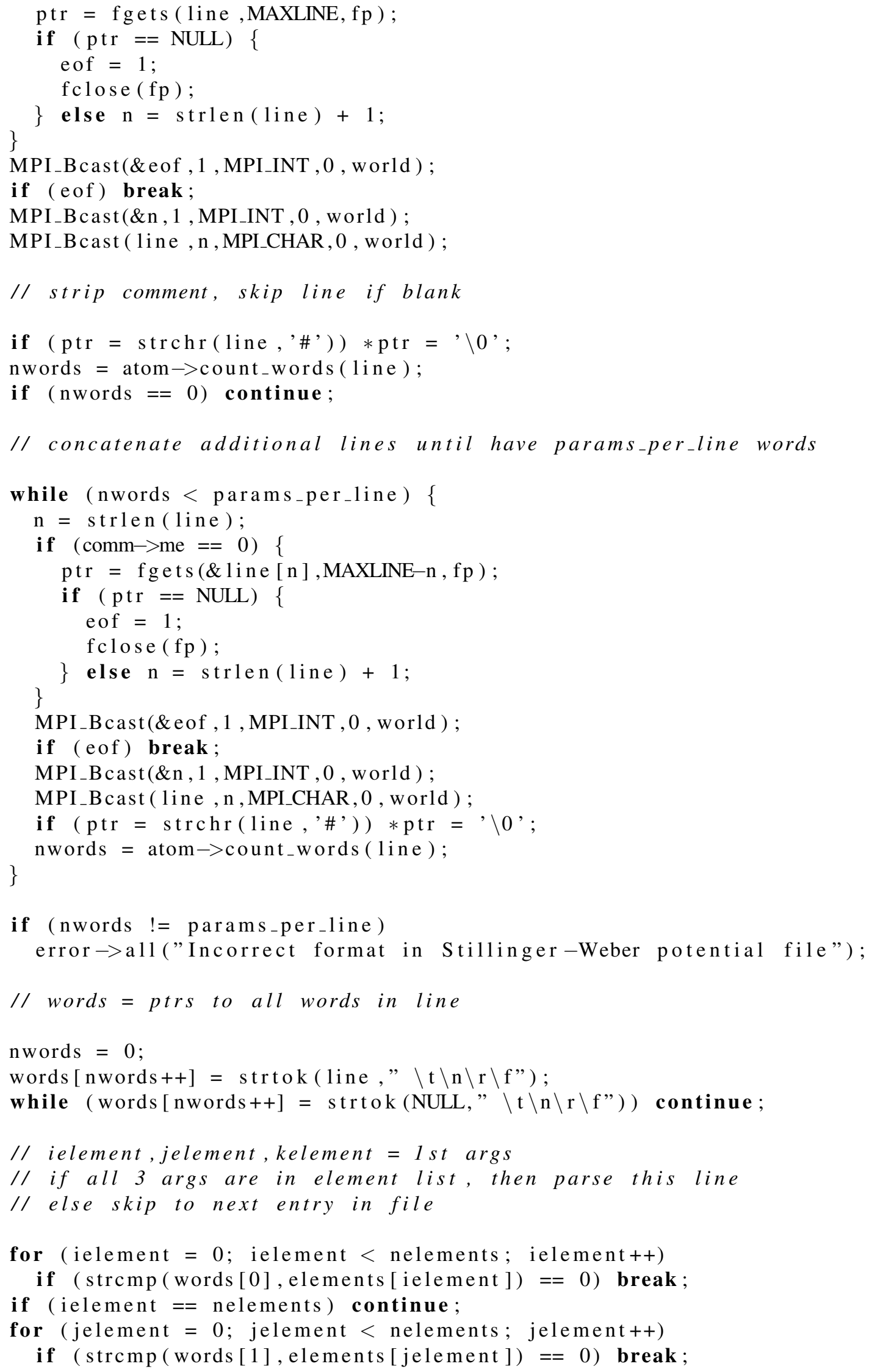




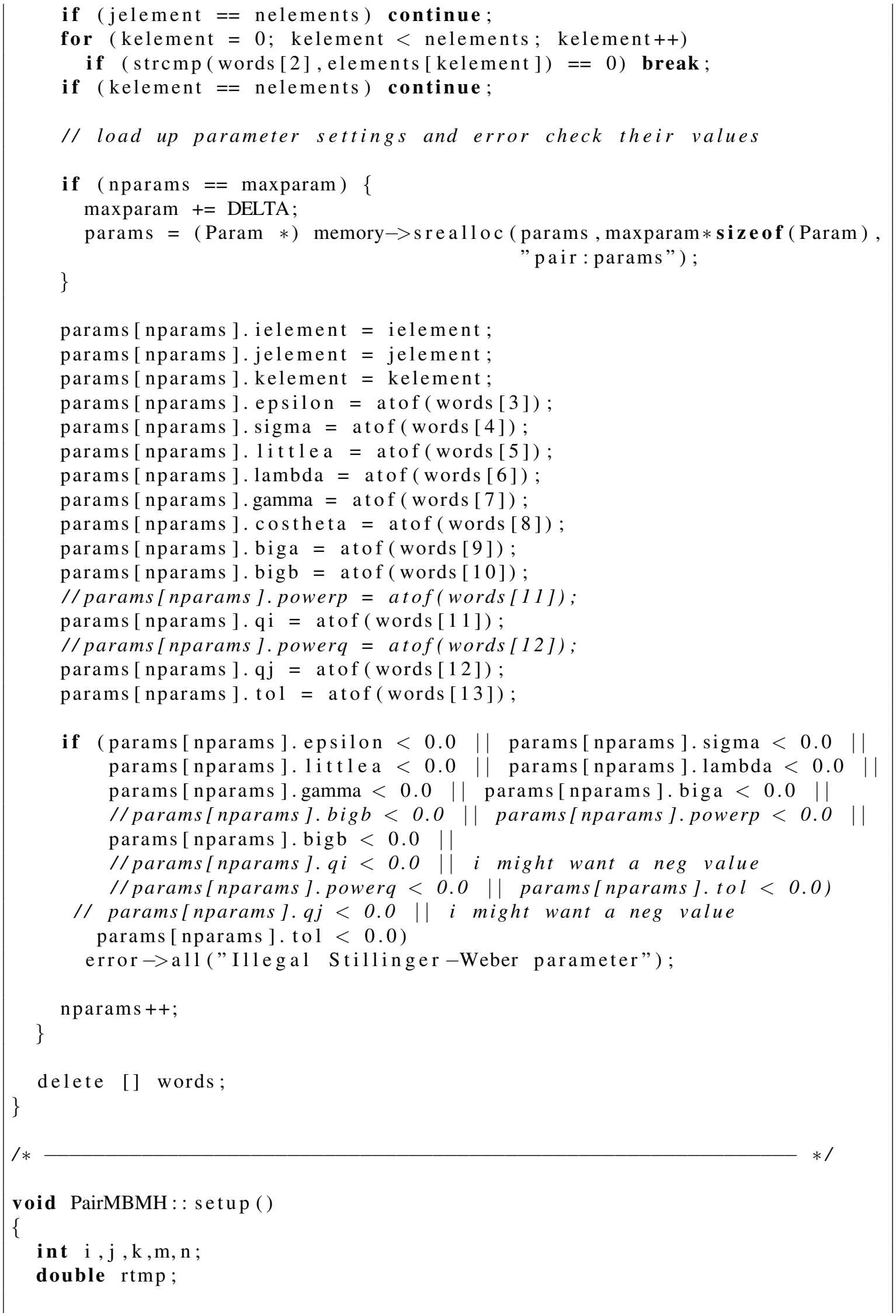




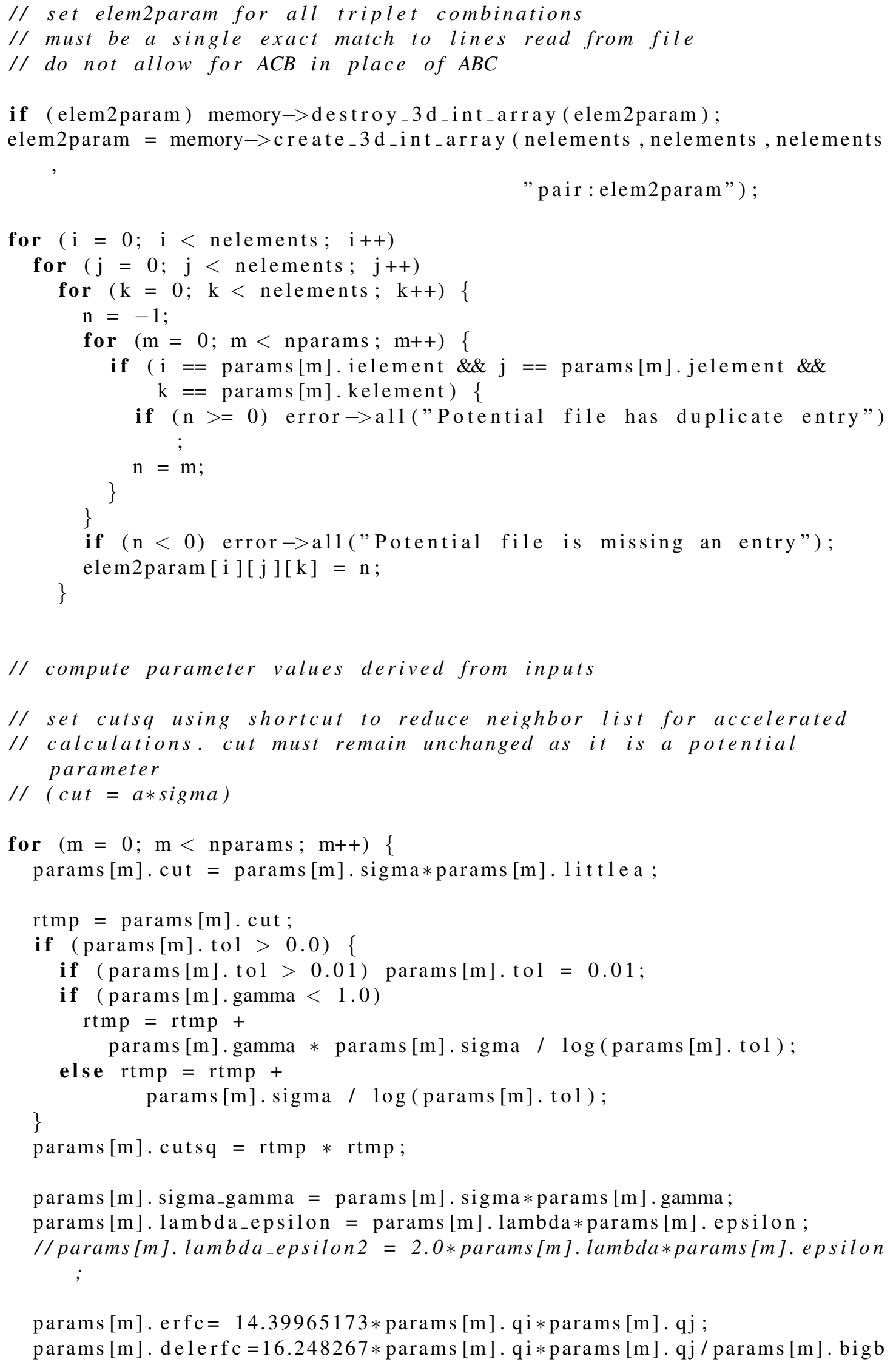




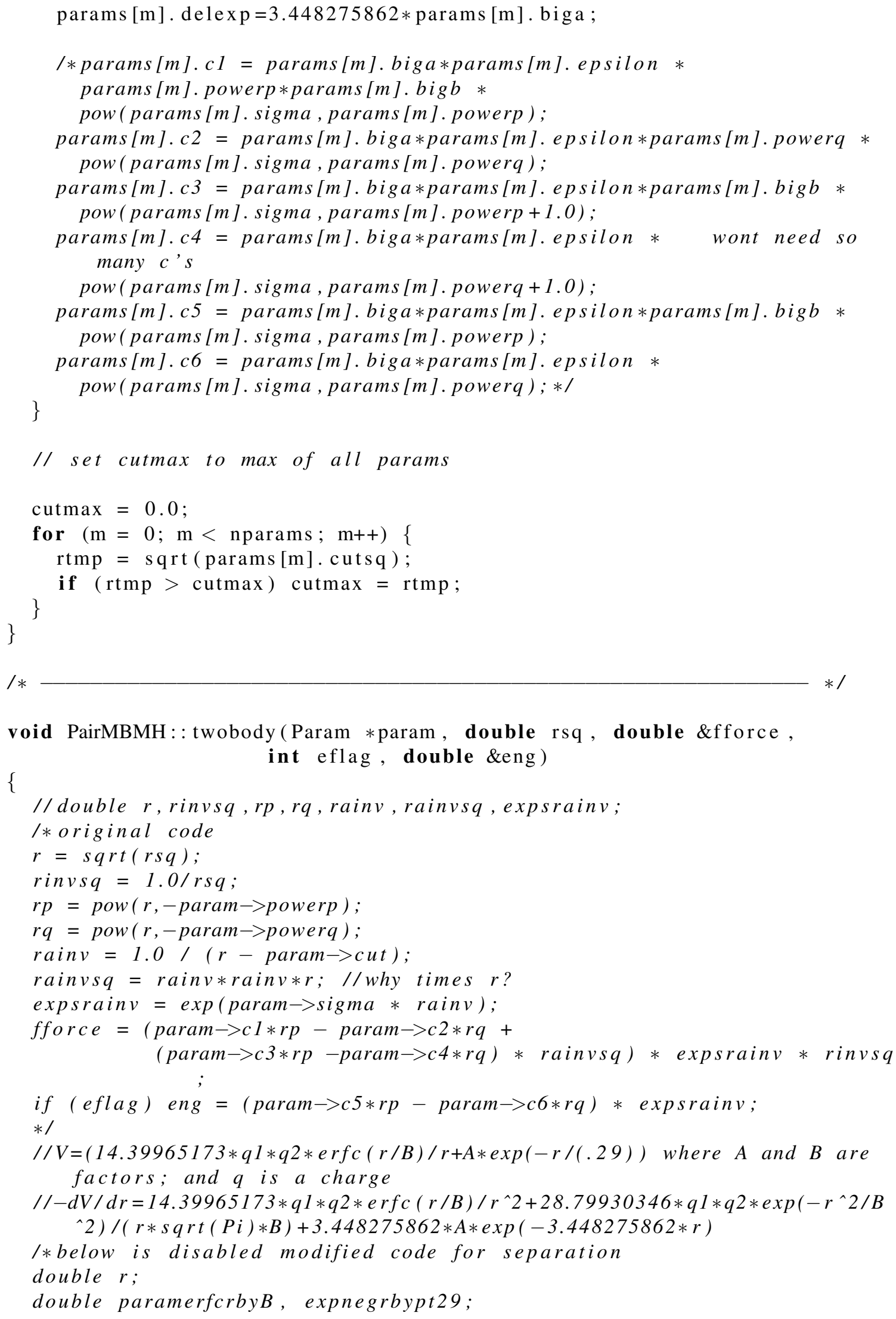




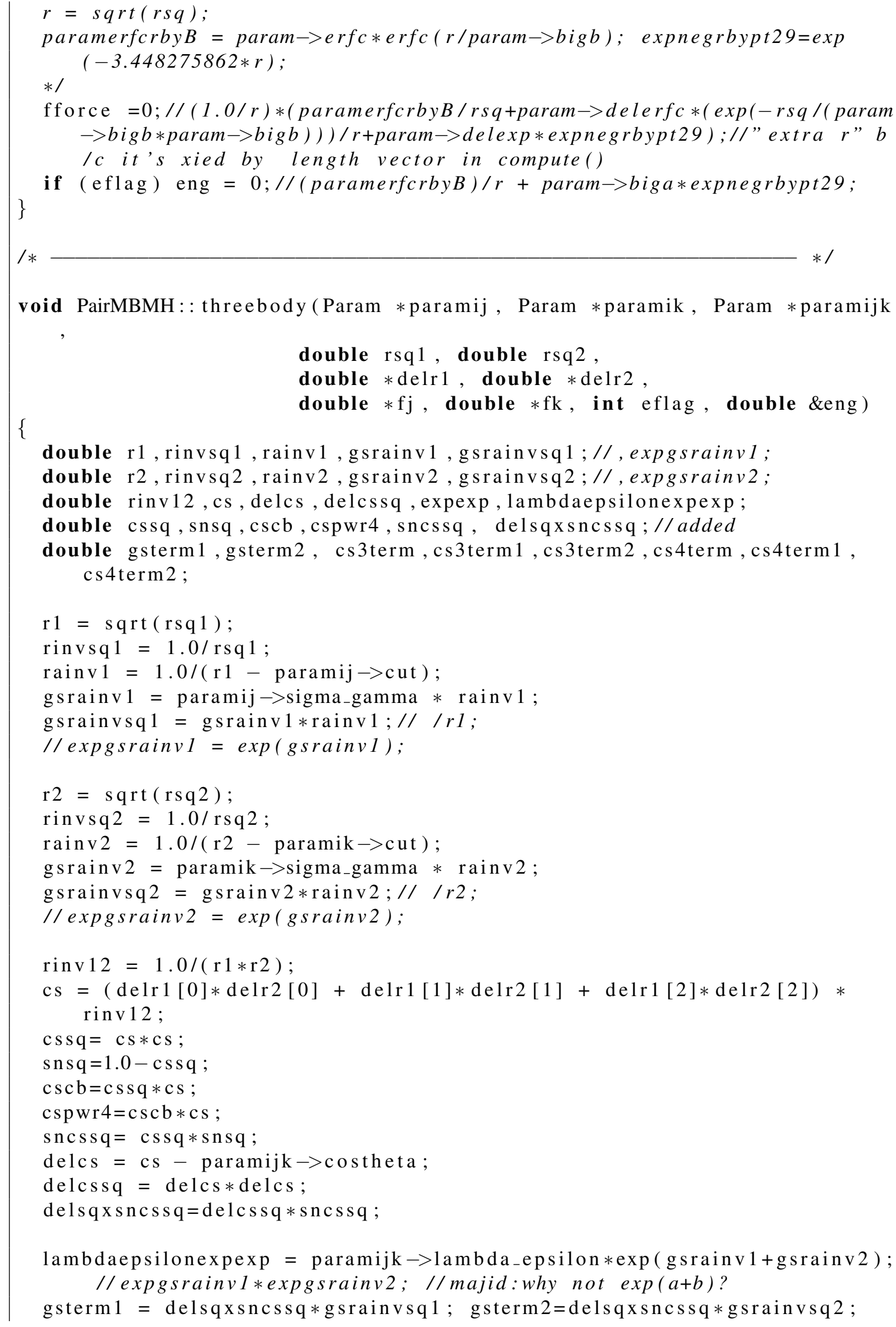




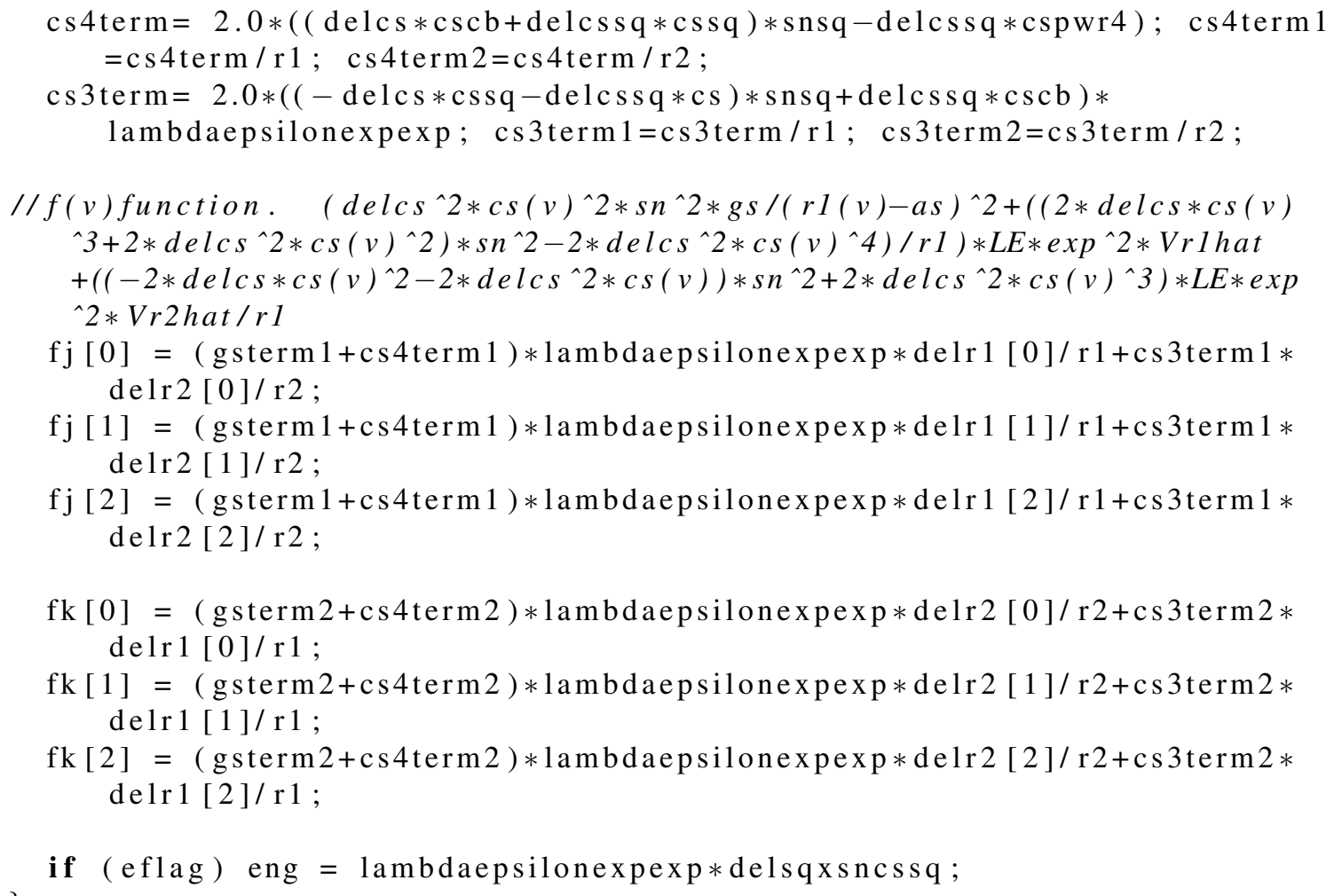

$\mathrm{fj}[0]=(\operatorname{gsterm} 1+\operatorname{cs} 4 \operatorname{term} 1) * \operatorname{lambdaepsilonexpexp} * \operatorname{de} \operatorname{lr} 1[0] / \mathrm{r} 1+\operatorname{cs} 3 \operatorname{term} 1 *$ delr2 $[0] / \mathrm{r} 2$

$\mathrm{fj}[1]=(\operatorname{gsterm} 1+\operatorname{cs} 4 \operatorname{term} 1) * \operatorname{lambdaepsilonexpexp} * \operatorname{del} 1 \mathrm{r}[1] / \mathrm{r} 1+\operatorname{cs} 3 \operatorname{term} 1 *$ delr2 [1]/r2 ;

$\mathrm{fj}[2]=(\mathrm{gsterm} 1+\operatorname{cs} 4 \operatorname{term} 1) * \operatorname{lambdaepsilonexpexp} * \operatorname{del} 1 \mathrm{r} 12] / \mathrm{r} 1+\operatorname{cs} 3 \operatorname{term} 1 *$ delr2 $[2] / \mathrm{r} 2$

$\mathrm{fk}[0]=(\operatorname{gsterm} 2+\operatorname{cs} 4 \operatorname{term} 2) * 1 \mathrm{ambdaepsilonexpexp} * \operatorname{delr} 2[0] / \mathrm{r} 2+\operatorname{cs} 3 \operatorname{term} 2 *$ delr1 $[0] / \mathrm{r} 1$;

$\mathrm{fk}[1]=(\mathrm{gsterm} 2+\operatorname{cs} 4 \operatorname{term} 2) * \operatorname{lambdaepsilonexpexp} * \operatorname{delr} 2[1] / \mathrm{r} 2+\operatorname{cs} 3 \operatorname{term} 2 *$ delr1 $[1] / \mathrm{r} 1$;

$\mathrm{fk}[2]=(\mathrm{gsterm} 2+\operatorname{cs} 4 \operatorname{term} 2) *$ lambdaepsilonexpexp $*$ delr $2[2] / \mathrm{r} 2+\operatorname{cs} 3 \operatorname{term} 2 *$ delr1 $[2] / \mathrm{r} 1$;

if (eflag) eng = lambdaepsilonexpexp*delsqxancssq; 
LAMMPS SOURCE MODIFICATION TEST

Simulation Setup Script

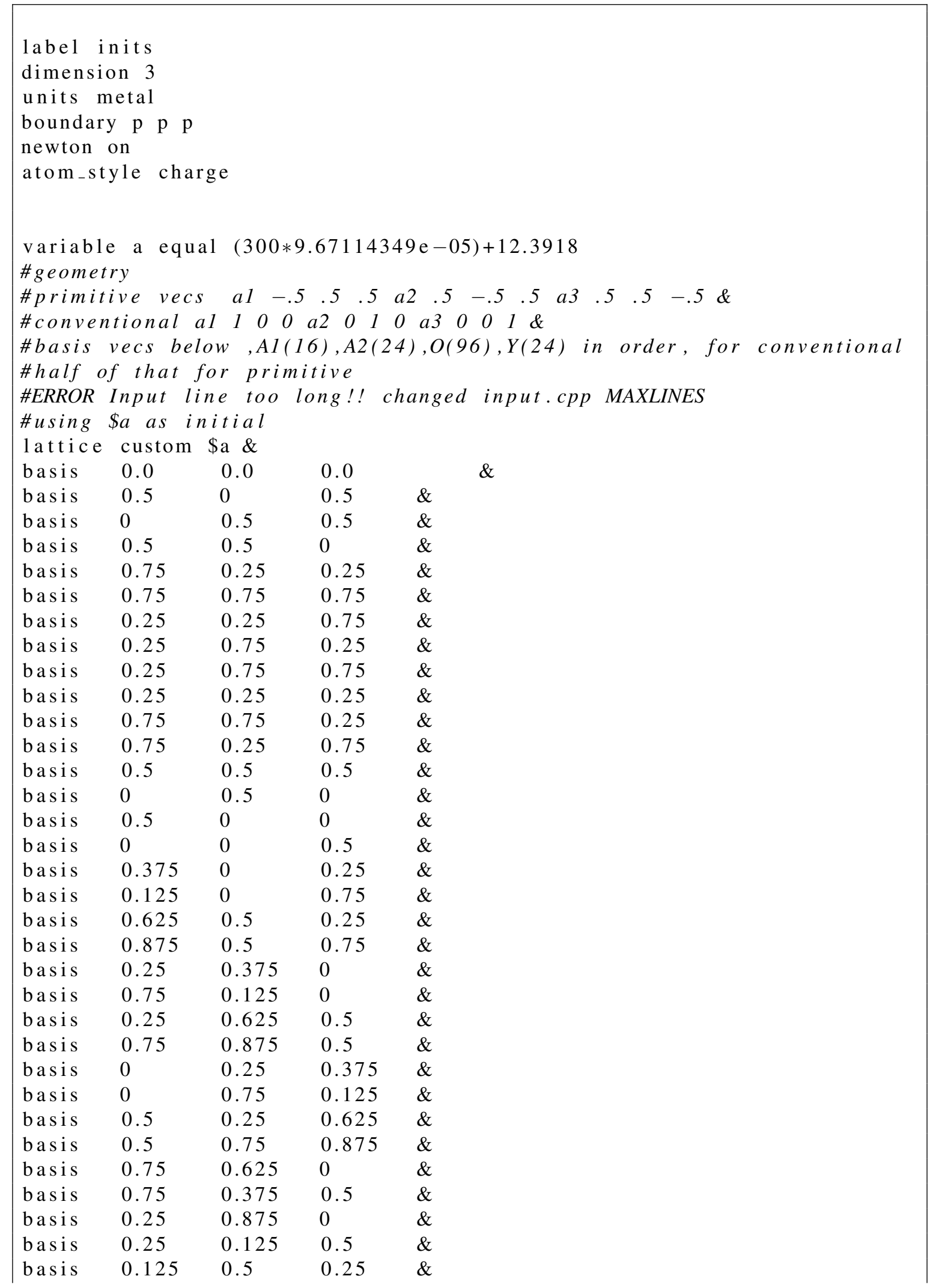




\begin{tabular}{|lllll} 
basis & 0.875 & 0 & 0.25 & $\&$ \\
basis & 0.375 & 0.5 & 0.75 & $\&$ \\
basis & 0.625 & 0 & 0.75 & $\&$ \\
basis & 0 & 0.25 & 0.875 & $\&$ \\
basis & 0.5 & 0.25 & 0.125 & $\&$ \\
basis & 0 & 0.75 & 0.625 & $\&$ \\
basis & 0.5 & 0.75 & 0.375 & $\&$ \\
basis & 0.28023 & 0.1011 & 0.19922 & $\&$ \\
basis & 0.21977 & 0.8989 & 0.69922 & $\&$ \\
basis & 0.71977 & 0.6011 & 0.30078 & $\&$ \\
basis & 0.78023 & 0.3989 & 0.80078 & $\&$ \\
basis & 0.19922 & 0.28023 & 0.1011 & $\&$ \\
basis & 0.69922 & 0.21977 & 0.8989 & $\&$ \\
basis & 0.30078 & 0.71977 & 0.6011 & $\&$ \\
basis & 0.80078 & 0.78023 & 0.3989 & $\&$ \\
basis & 0.1011 & 0.19922 & 0.28023 & $\&$ \\
basis & 0.8989 & 0.69922 & 0.21977 & $\&$ \\
basis & 0.6011 & 0.30078 & 0.71977 & $\&$ \\
basis & 0.3989 & 0.80078 & 0.78023 & $\&$ \\
basis & 0.8511 & 0.53023 & 0.05078 & $\&$ \\
basis & 0.6489 & 0.46977 & 0.55078 & $\&$ \\
basis & 0.3511 & 0.96977 & 0.94922 & $\&$ \\
basis & 0.1489 & 0.03023 & 0.44922 & $\&$ \\
basis & 0.03023 & 0.44922 & 0.1489 & $\&$ \\
basis & 0.96977 & 0.94922 & 0.3511 & $\&$ \\
basis & 0.46977 & 0.55078 & 0.6489 & $\&$ \\
basis & 0.53023 & 0.05078 & 0.8511 & $\&$ \\
basis & 0.94922 & 0.3511 & 0.96977 & $\&$ \\
basis & 0.44922 & 0.1489 & 0.03023 & $\&$ \\
basis & 0.05078 & 0.8511 & 0.53023 & $\&$ \\
basis & 0.55078 & 0.6489 & 0.46977 & $\&$ \\
basis & 0.71977 & 0.8989 & 0.80078 & $\&$ \\
basis & 0.78023 & 0.1011 & 0.30078 & $\&$ \\
basis & 0.28023 & 0.3989 & 0.69922 & $\&$ \\
basis & 0.21977 & 0.6011 & 0.19922 & $\&$ \\
basis & 0.80078 & 0.71977 & 0.8989 & $\&$ \\
basis & 0.30078 & 0.78023 & 0.1011 & $\&$ \\
basis & 0.69922 & 0.28023 & 0.3989 & $\&$ \\
basis & 0.19922 & 0.21977 & 0.6011 & $\&$ \\
basis & 0.8989 & 0.80078 & 0.71977 & $\&$ \\
basis & 0.1011 & 0.30078 & 0.78023 & $\&$ \\
basis & 0.3989 & 0.69922 & 0.28023 & $\&$ \\
basis & 0.6011 & 0.19922 & 0.21977 & $\&$ \\
basis & 0.1489 & 0.46977 & 0.94922 & $\&$ \\
basis & 0.3511 & 0.53023 & 0.44922 & $\&$ \\
basis & 0.6489 & 0.03023 & 0.05078 & $\&$ \\
basis & 0.8511 & 0.96977 & 0.55078 & $\&$ \\
basis & 0.96977 & 0.55078 & 0.8511 & $\&$ \\
basis & 0.03023 & 0.05078 & 0.6489 & $\&$ \\
basis & 0.53023 & 0.44922 & 0.3511 & $\&$ \\
basis & 0.46977 & 0.94922 & 0.1489 & $\&$ \\
basis & 0.05078 & 0.6489 & 0.03023 & $\&$ \\
basis & 0.55078 & 0.8511 & 0.96977 & $\&$ \\
basis & 0.94922 & 0.1489 & 0.46977 & $\&$
\end{tabular}




\begin{tabular}{|c|c|c|c|}
\hline bas is & 0.44922 & & \\
\hline basis & 0.78023 & 0.6011 & 0.69922 \\
\hline oasis & 0.71977 & 0.3989 & 0.19922 \\
\hline asis & 0.21977 & 0.1011 & 0.80078 \\
\hline asis & 0.28023 & 0.8989 & 0.30078 \\
\hline sis & 0.69922 & 0.78023 & 0.6011 \\
\hline asis & 0.19922 & 0.71977 & \\
\hline sis & 0.80078 & 0.21977 & \\
\hline s is & 0.30078 & 0.28023 & \\
\hline is is & & 0.69922 & \\
\hline sis & & & \\
\hline asis & & & \\
\hline asis & & & \\
\hline sis & & & \\
\hline sis & & & \\
\hline as is & & & \\
\hline is is & & & \\
\hline is is & & & \\
\hline $\mathrm{sis}$ & & 0.4 & \\
\hline sis & & & \\
\hline sis & & & \\
\hline sis & & & \\
\hline sis & & & \\
\hline sis & & & \\
\hline s is & & & \\
\hline $\mathrm{sis}$ & & & \\
\hline is is & 23 & 0.6 & 78 \\
\hline is is & 23 & & 22 \\
\hline sis & 77 & 0.1 & 922 \\
\hline is is & & 0.2 & \\
\hline s is & & 0.2 & \\
\hline sis & & 0.7 & \\
\hline sis & 22 & 0.7 & \\
\hline sis & & 0.3 & \\
\hline sis & 1 & 0.8 & 023 \\
\hline sis & & 0.1 & 023 \\
\hline $\mathrm{sis}$ & 11 & 0.6 & 977 \\
\hline is is & 9 & 0.9 & 922 \\
\hline sis & 11 & 0.0 & 922 \\
\hline asis & 9 & 0.5 & 0.55078 \\
\hline asis & 1 & 0.46977 & 0.05078 \\
\hline basis & 977 & 5078 & \\
\hline basis & 023 & 5078 & \\
\hline basis & 023 & 4922 & \\
\hline basis & 977 & 0.44922 & \\
\hline basis & 55078 & 0.1 & 023 \\
\hline basis & 05078 & 0.3 & \\
\hline sis & .44922 & 0.6 & 977 \\
\hline & .94922 & 0.8511 & 0.03023 \\
\hline & & 0 & \\
\hline & & 0 & \\
\hline & & 0 . & \\
\hline & & 0.5 & 0.75 \\
\hline & 0.25 & 0.125 & \\
\hline
\end{tabular}




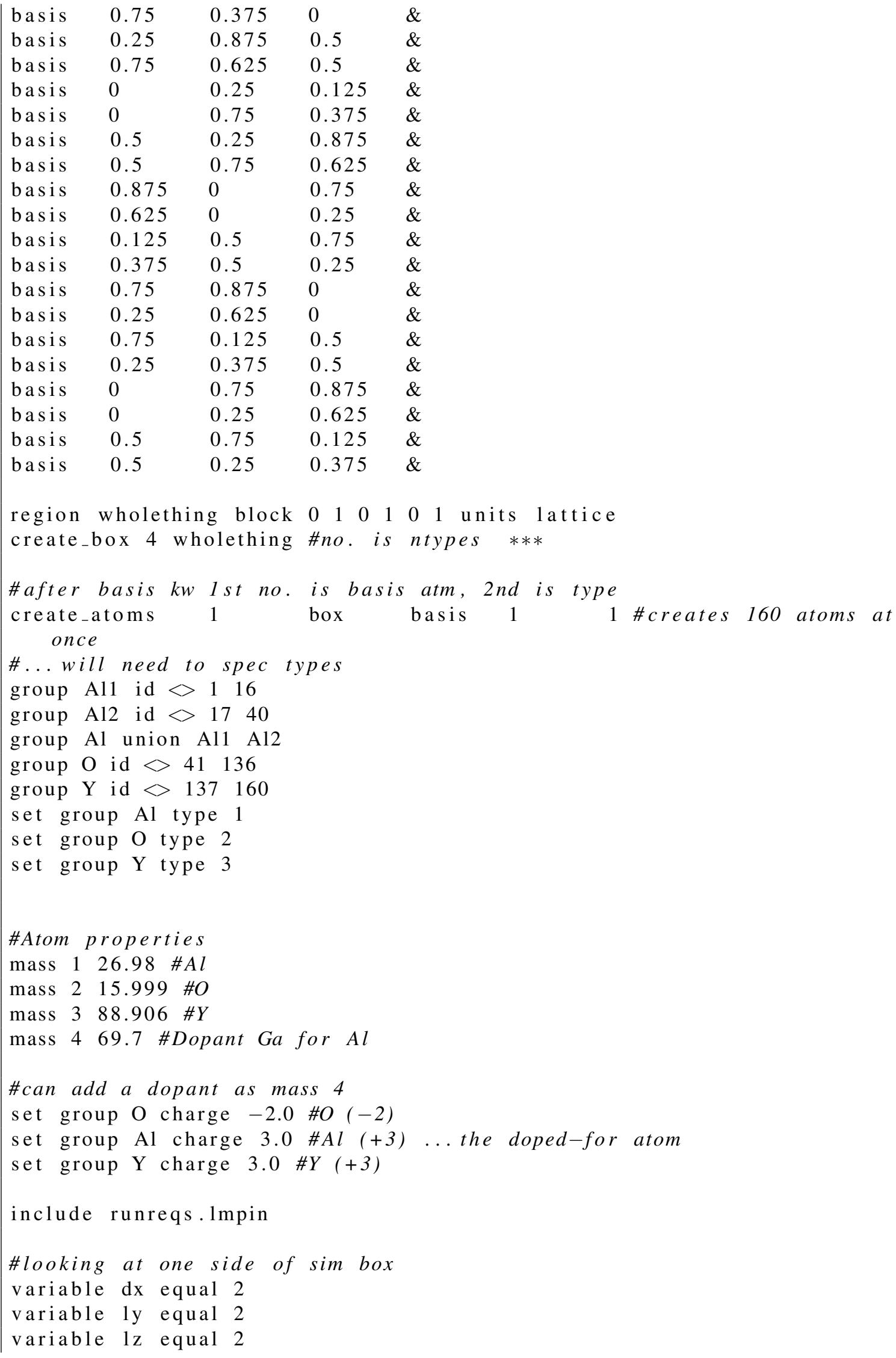




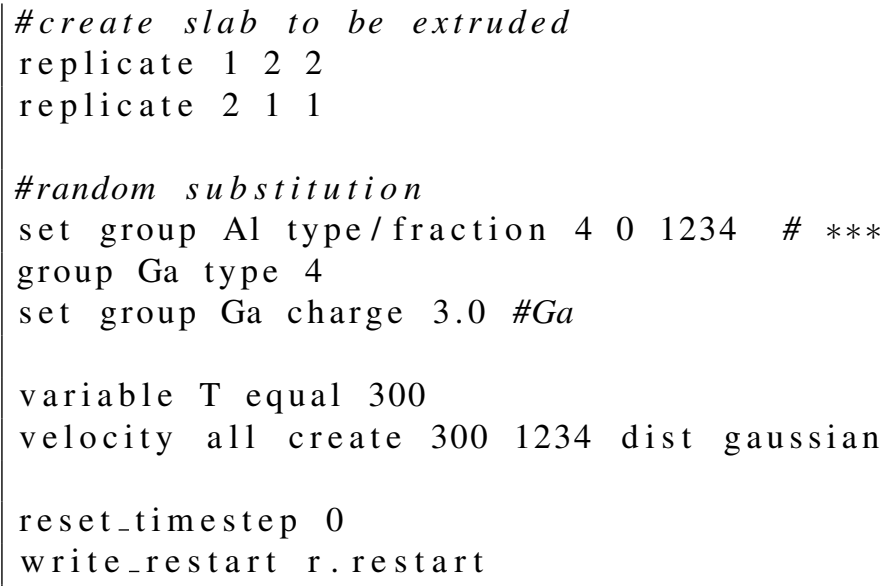

\section{Interatomic Potentials}

\section{runreqs.lmpin}

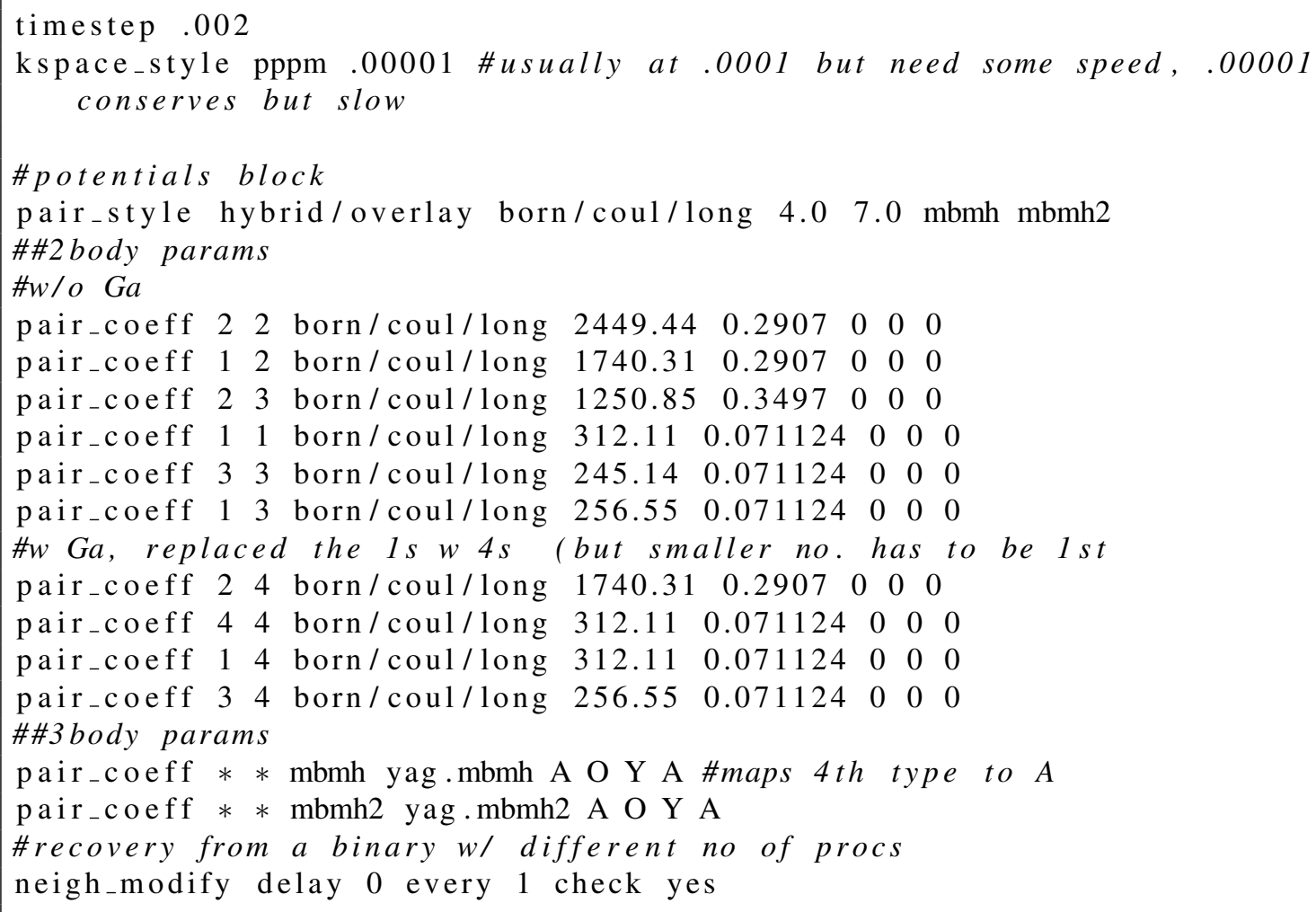

3-Body parameters (yag.mbmh)

\#3body only for certain kinds and only for XYY

\# $(a *(\operatorname{sigma}=1))$ is rijO, lambda*(epsilon=1) is just lambda \# KEEP IT 1 FOR ALL EVEN IF NO THREE BODY INTERACTION!

$\# b / c$ same param used in 2 body and we dont want to zero that

$\begin{array}{ccccccccc}\text { A A A } 1.0 & 1.0 & 5.0 & 0.0 & 0.0 & -0.333333333333 & \text { \#cut@ } 5 \\ & & 312.11 & 14.06 & 3.0 & 3.0 & 0.0\end{array}$ 


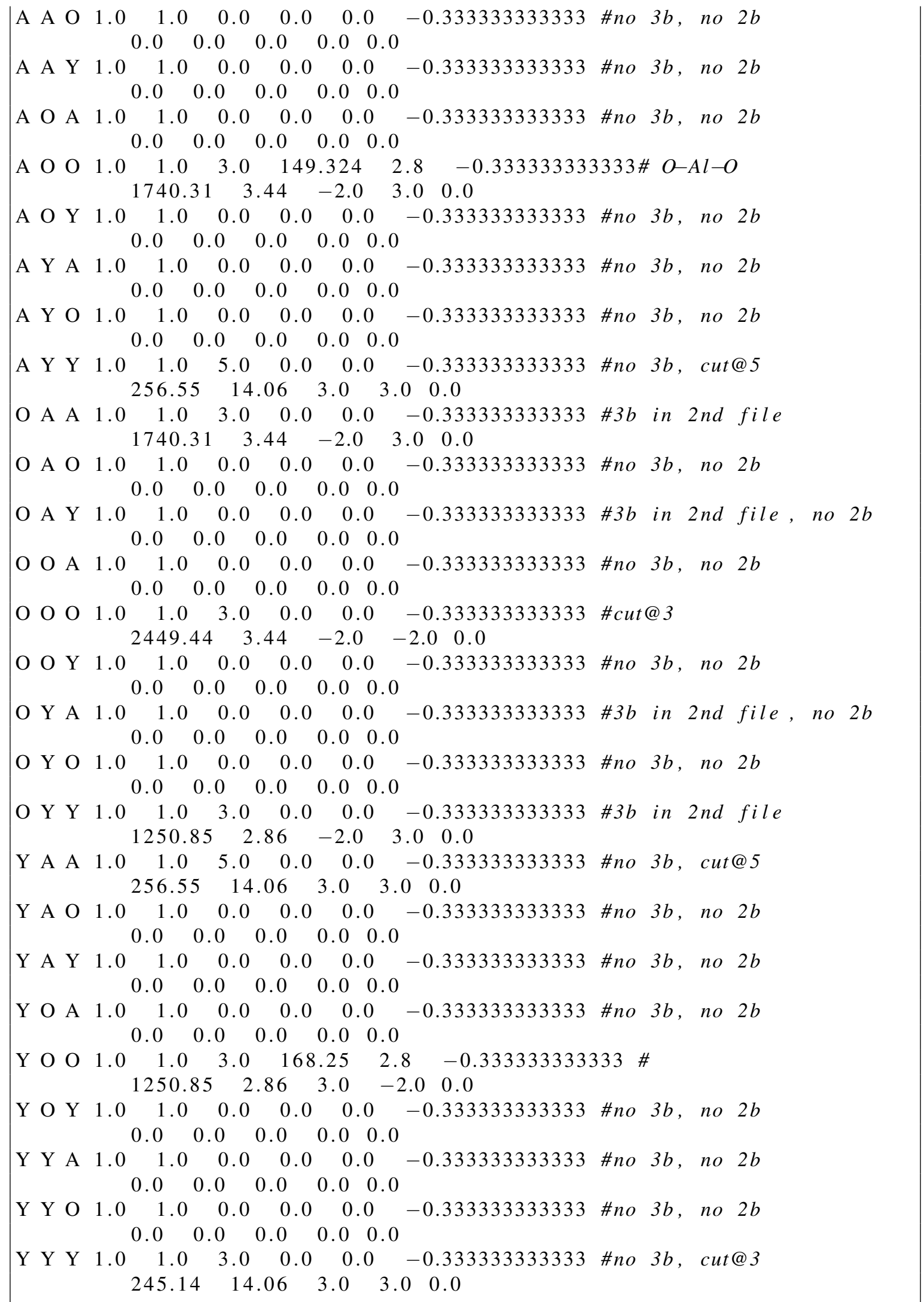

3-Body parameters (yag.mbmh2) 


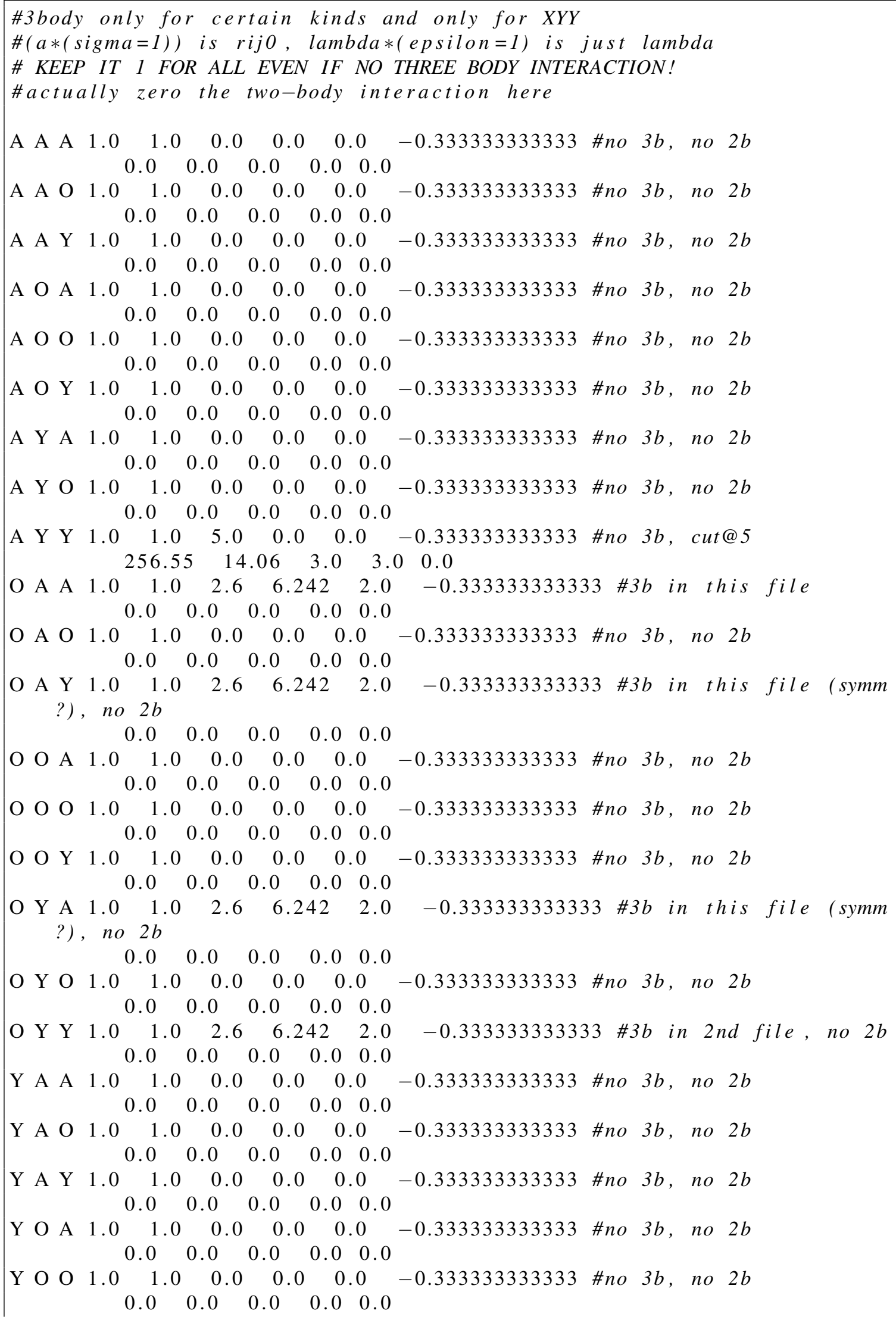




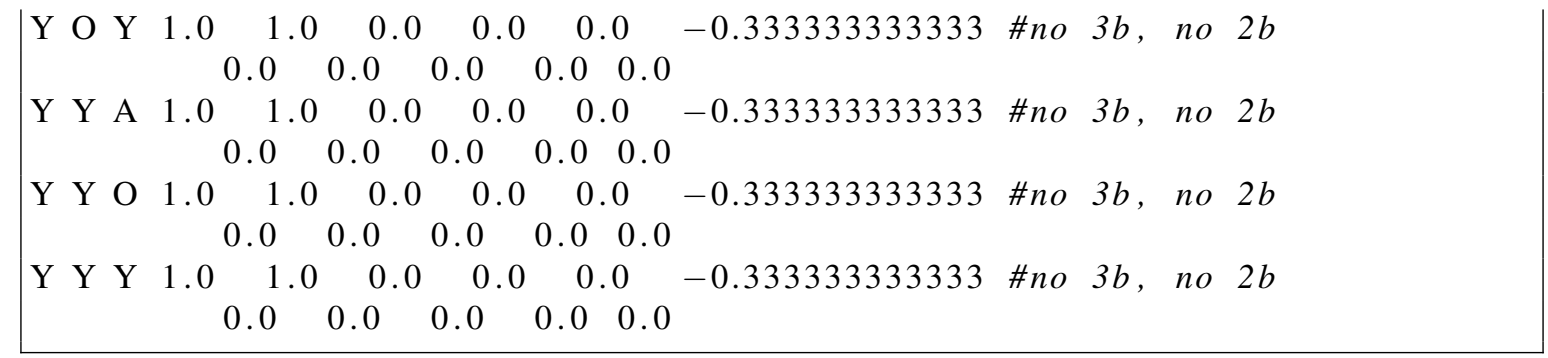

\section{Simulation Execution}

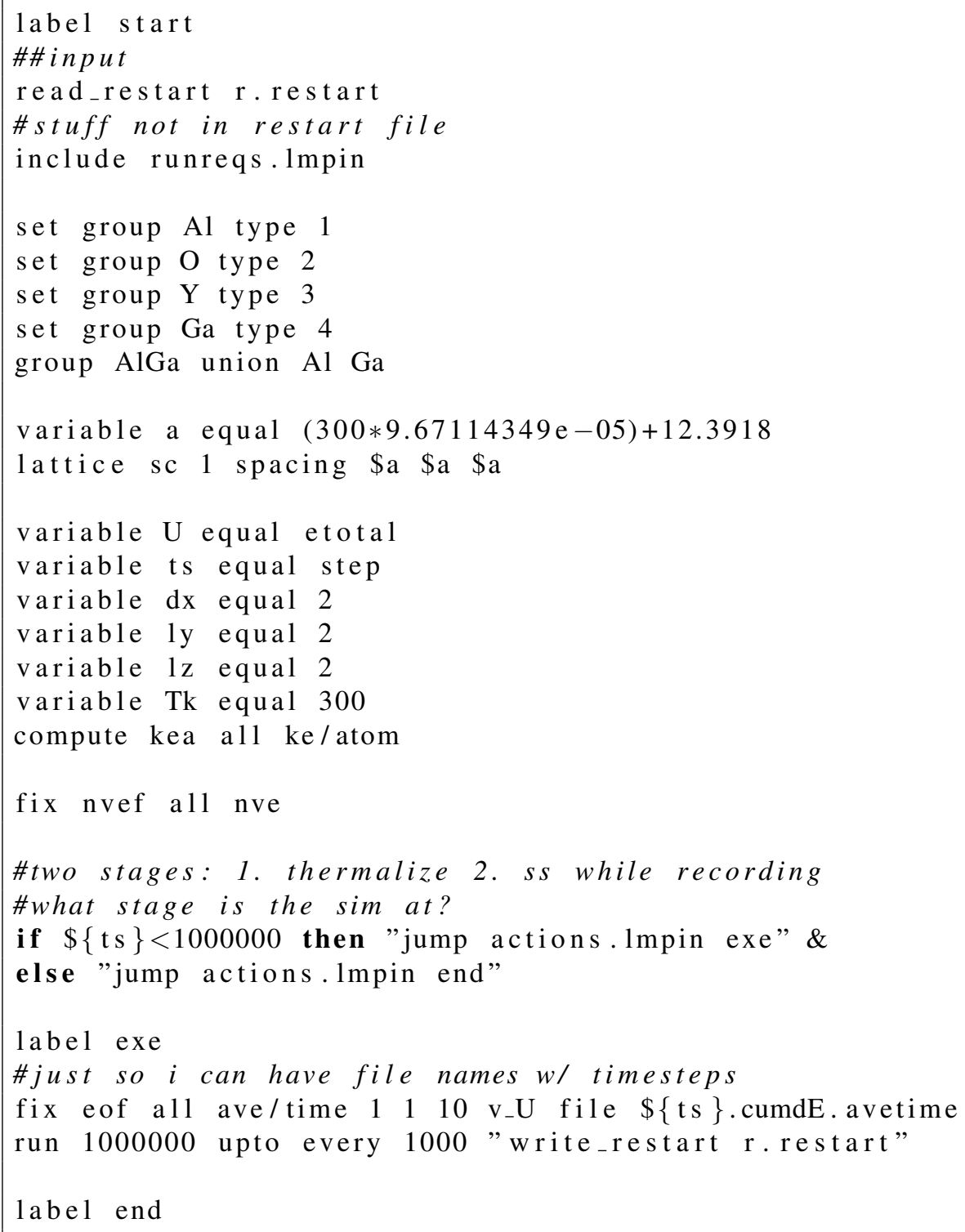




\section{LAMMPS SCRIPTS FOR LATTICE CONSTANT, SPECIFIC HEAT, THERMAL EXPANSION}

\section{Simulation Setup}

This script sets up the simulation.

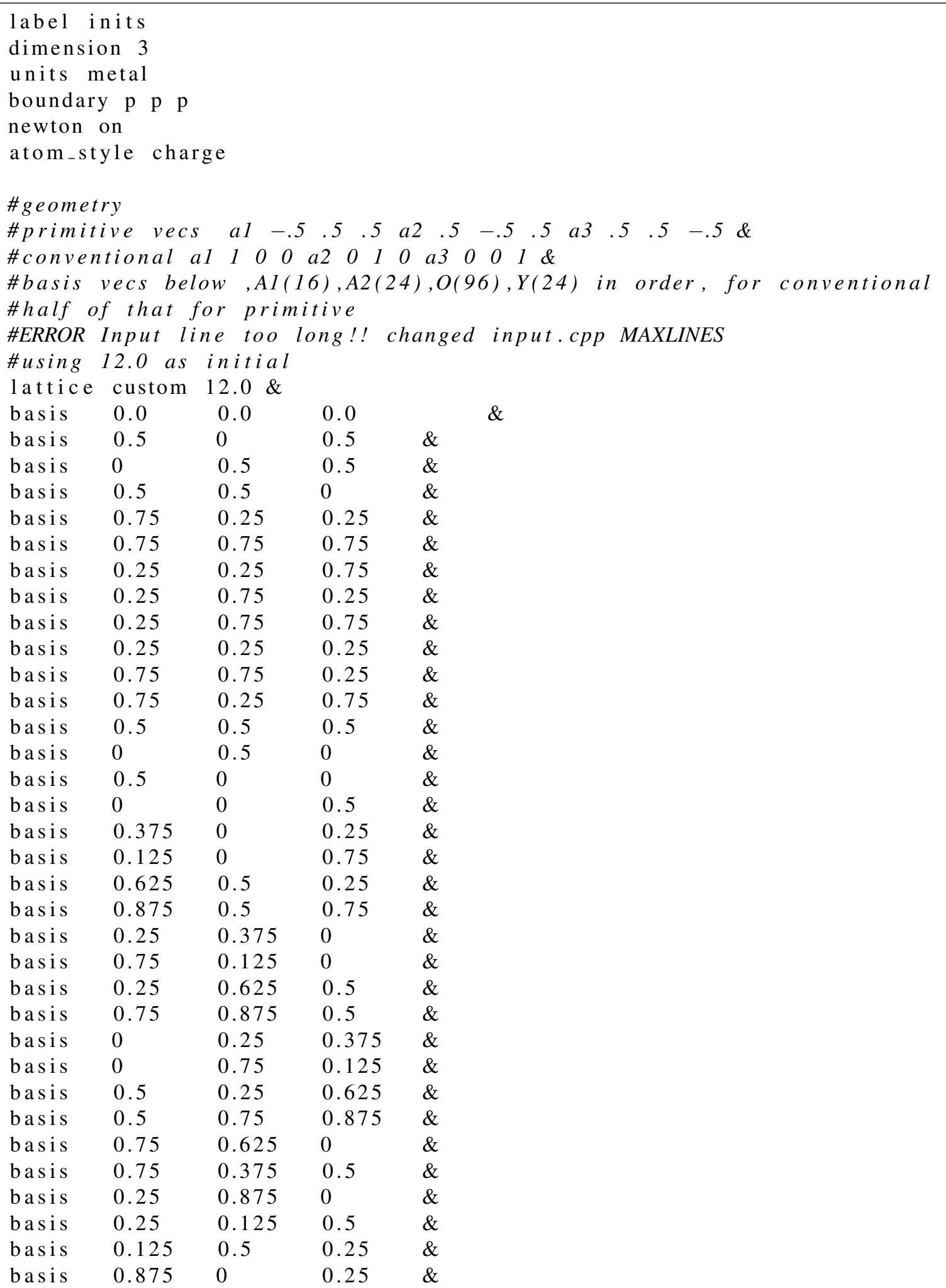




\begin{tabular}{|lllll} 
basis & 0.375 & 0.5 & 0.75 & $\&$ \\
basis & 0.625 & 0 & 0.75 & $\&$ \\
basis & 0 & 0.25 & 0.875 & $\&$ \\
basis & 0.5 & 0.25 & 0.125 & $\&$ \\
basis & 0 & 0.75 & 0.625 & $\&$ \\
basis & 0.5 & 0.75 & 0.375 & $\&$ \\
basis & 0.28023 & 0.1011 & 0.19922 & $\&$ \\
basis & 0.21977 & 0.8989 & 0.69922 & $\&$ \\
basis & 0.71977 & 0.6011 & 0.30078 & $\&$ \\
basis & 0.78023 & 0.3989 & 0.80078 & $\&$ \\
basis & 0.19922 & 0.28023 & 0.1011 & $\&$ \\
basis & 0.69922 & 0.21977 & 0.8989 & $\&$ \\
basis & 0.30078 & 0.71977 & 0.6011 & $\&$ \\
basis & 0.80078 & 0.78023 & 0.3989 & $\&$ \\
basis & 0.1011 & 0.19922 & 0.28023 & $\&$ \\
basis & 0.8989 & 0.69922 & 0.21977 & $\&$ \\
basis & 0.6011 & 0.30078 & 0.71977 & $\&$ \\
basis & 0.3989 & 0.80078 & 0.78023 & $\&$ \\
basis & 0.8511 & 0.53023 & 0.05078 & $\&$ \\
basis & 0.6489 & 0.46977 & 0.55078 & $\&$ \\
basis & 0.3511 & 0.96977 & 0.94922 & $\&$ \\
basis & 0.1489 & 0.03023 & 0.44922 & $\&$ \\
basis & 0.03023 & 0.44922 & 0.1489 & $\&$ \\
basis & 0.96977 & 0.94922 & 0.3511 & $\&$ \\
basis & 0.46977 & 0.55078 & 0.6489 & $\&$ \\
basis & 0.53023 & 0.05078 & 0.8511 & $\&$ \\
basis & 0.94922 & 0.3511 & 0.96977 & $\&$ \\
basis & 0.44922 & 0.1489 & 0.03023 & $\&$ \\
basis & 0.05078 & 0.8511 & 0.53023 & $\&$ \\
basis & 0.55078 & 0.6489 & 0.46977 & $\&$ \\
basis & 0.71977 & 0.8989 & 0.80078 & $\&$ \\
basis & 0.78023 & 0.1011 & 0.30078 & $\&$ \\
basis & 0.28023 & 0.3989 & 0.69922 & $\&$ \\
basis & 0.21977 & 0.6011 & 0.19922 & $\&$ \\
basis & 0.80078 & 0.71977 & 0.8989 & $\&$ \\
basis & 0.30078 & 0.78023 & 0.1011 & $\&$ \\
basis & 0.69922 & 0.28023 & 0.3989 & $\&$ \\
basis & 0.19922 & 0.21977 & 0.6011 & $\&$ \\
basis & 0.8989 & 0.80078 & 0.71977 & $\&$ \\
basis & 0.1011 & 0.30078 & 0.78023 & $\&$ \\
basis & 0.3989 & 0.69922 & 0.28023 & $\&$ \\
basis & 0.6011 & 0.19922 & 0.21977 & $\&$ \\
basis & 0.1489 & 0.46977 & 0.94922 & $\&$ \\
basis & 0.3511 & 0.53023 & 0.44922 & $\&$ \\
basis & 0.6489 & 0.03023 & 0.05078 & $\&$ \\
basis & 0.8511 & 0.96977 & 0.55078 & $\&$ \\
basis & 0.96977 & 0.55078 & 0.8511 & $\&$ \\
basis & 0.03023 & 0.05078 & 0.6489 & $\&$ \\
basis & 0.53023 & 0.44922 & 0.3511 & $\&$ \\
basis & 0.46977 & 0.94922 & 0.1489 & $\&$ \\
basis & 0.05078 & 0.6489 & 0.03023 & $\&$ \\
basis & 0.55078 & 0.8511 & 0.96977 & $\&$ \\
basis & 0.94922 & 0.1489 & 0.46977 & $\&$ \\
basis & 0.44922 & 0.3511 & 0.53023 & $\&$
\end{tabular}




\begin{tabular}{|lllll} 
basis & 0.78023 & 0.6011 & 0.69922 & $\&$ \\
basis & 0.71977 & 0.3989 & 0.19922 & $\&$ \\
basis & 0.21977 & 0.1011 & 0.80078 & $\&$ \\
basis & 0.28023 & 0.8989 & 0.30078 & $\&$ \\
basis & 0.69922 & 0.78023 & 0.6011 & $\&$ \\
basis & 0.19922 & 0.71977 & 0.3989 & $\&$ \\
basis & 0.80078 & 0.21977 & 0.1011 & $\&$ \\
basis & 0.30078 & 0.28023 & 0.8989 & $\&$ \\
basis & 0.6011 & 0.69922 & 0.78023 & $\&$ \\
basis & 0.3989 & 0.19922 & 0.71977 & $\&$ \\
basis & 0.1011 & 0.80078 & 0.21977 & $\&$ \\
basis & 0.8989 & 0.30078 & 0.28023 & $\&$ \\
basis & 0.3511 & 0.03023 & 0.55078 & $\&$ \\
basis & 0.1489 & 0.96977 & 0.05078 & $\&$ \\
basis & 0.8511 & 0.46977 & 0.44922 & $\&$ \\
basis & 0.6489 & 0.53023 & 0.94922 & $\&$ \\
basis & 0.53023 & 0.94922 & 0.6489 & $\&$ \\
basis & 0.46977 & 0.44922 & 0.8511 & $\&$ \\
basis & 0.96977 & 0.05078 & 0.1489 & $\&$ \\
basis & 0.03023 & 0.55078 & 0.3511 & $\&$ \\
basis & 0.44922 & 0.8511 & 0.46977 & $\&$ \\
basis & 0.94922 & 0.6489 & 0.53023 & $\&$ \\
basis & 0.55078 & 0.3511 & 0.03023 & $\&$ \\
basis & 0.05078 & 0.1489 & 0.96977 & $\&$ \\
basis & 0.21977 & 0.3989 & 0.30078 & $\&$ \\
basis & 0.28023 & 0.6011 & 0.80078 & $\&$ \\
basis & 0.78023 & 0.8989 & 0.19922 & $\&$ \\
basis & 0.71977 & 0.1011 & 0.69922 & $\&$ \\
basis & 0.30078 & 0.21977 & 0.3989 & $\&$ \\
basis & 0.80078 & 0.28023 & 0.6011 & $\&$ \\
basis & 0.19922 & 0.78023 & 0.8989 & $\&$ \\
basis & 0.69922 & 0.71977 & 0.1011 & $\&$ \\
basis & 0.3989 & 0.30078 & 0.21977 & $\&$ \\
basis & 0.6011 & 0.80078 & 0.28023 & $\&$ \\
basis & 0.8989 & 0.19922 & 0.78023 & $\&$ \\
basis & 0.1011 & 0.69922 & 0.71977 & $\&$ \\
basis & 0.6489 & 0.96977 & 0.44922 & $\&$ \\
basis & 0.8511 & 0.03023 & 0.94922 & $\&$ \\
basis & 0.1489 & 0.53023 & 0.55078 & $\&$ \\
basis & 0.3511 & 0.46977 & 0.05078 & $\&$ \\
basis & 0.46977 & 0.05078 & 0.3511 & $\&$ \\
basis & 0.53023 & 0.55078 & 0.1489 & $\&$ \\
basis & 0.03023 & 0.94922 & 0.8511 & $\&$ \\
basis & 0.96977 & 0.44922 & 0.6489 & $\&$ \\
basis & 0.55078 & 0.1489 & 0.53023 & $\&$ \\
basis & 0.05078 & 0.3511 & 0.46977 & $\&$ \\
basis & 0.44922 & 0.6489 & 0.96977 & $\&$ \\
basis & 0.94922 & 0.8511 & 0.03023 & $\&$ \\
basis & 0.125 & 0 & 0.25 & $\&$ \\
basis & 0.375 & 0 & 0.75 & $\&$ \\
basis & 0.875 & 0.5 & 0.25 & $\&$ \\
basis & 0.625 & 0.5 & 0.75 & $\&$ \\
basis & 0.25 & 0.125 & 0 & $\&$ \\
basis & 0.75 & 0.375 & 0 & $\&$
\end{tabular}




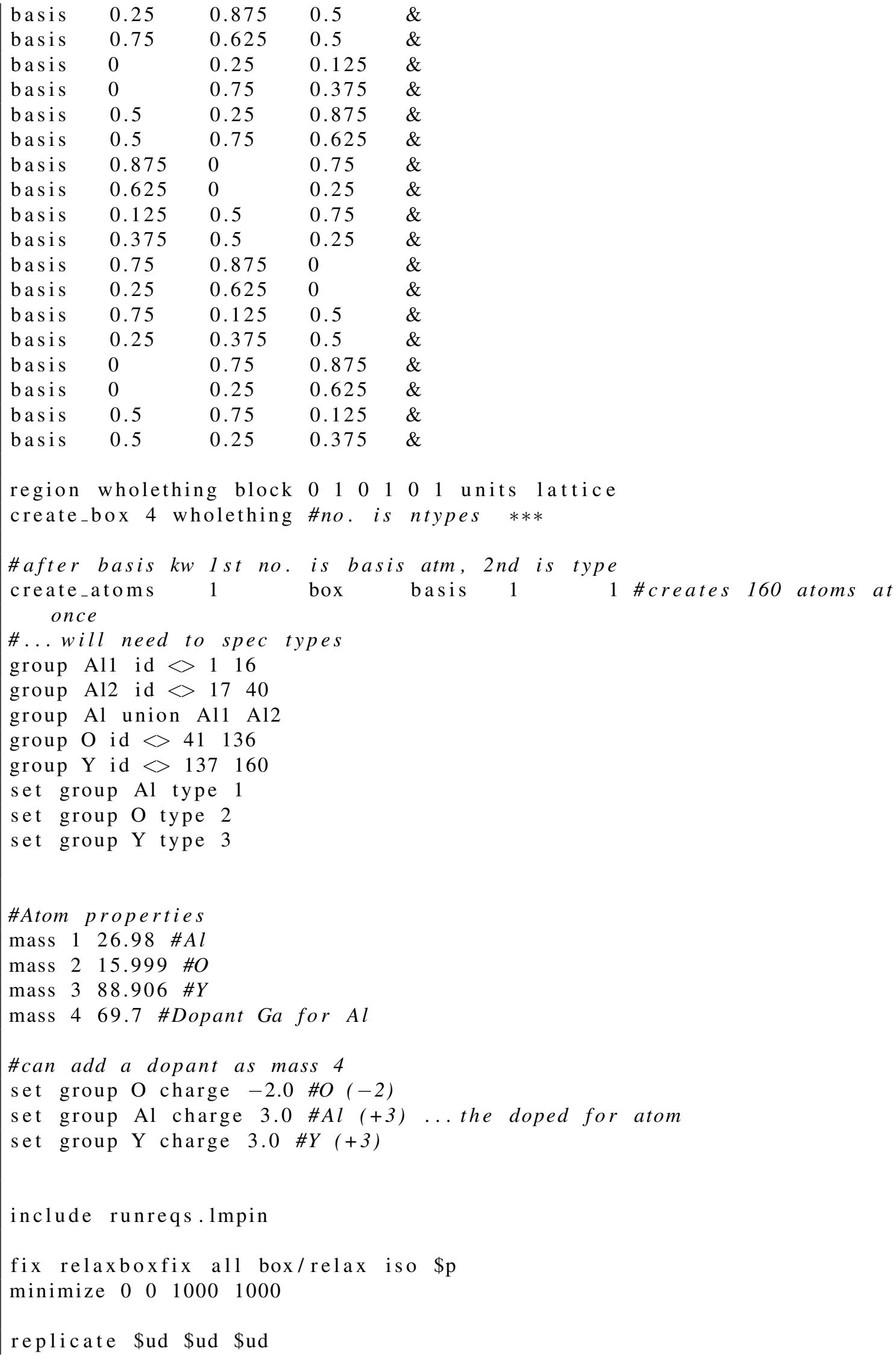


\#random doping

set group Al type/fraction 4 \$dp \$dseed \#***

group Ga type 4

set group Ga charge $3.0 \# G a$

variable T0 equal 10

velocity all create $\$\{\mathrm{~T} 0\}$ $\$$ vseed dist gaussian

reset_timestep 0

write_restart r. restart

\section{Interatomic Potentials}

runreqs.lmpin

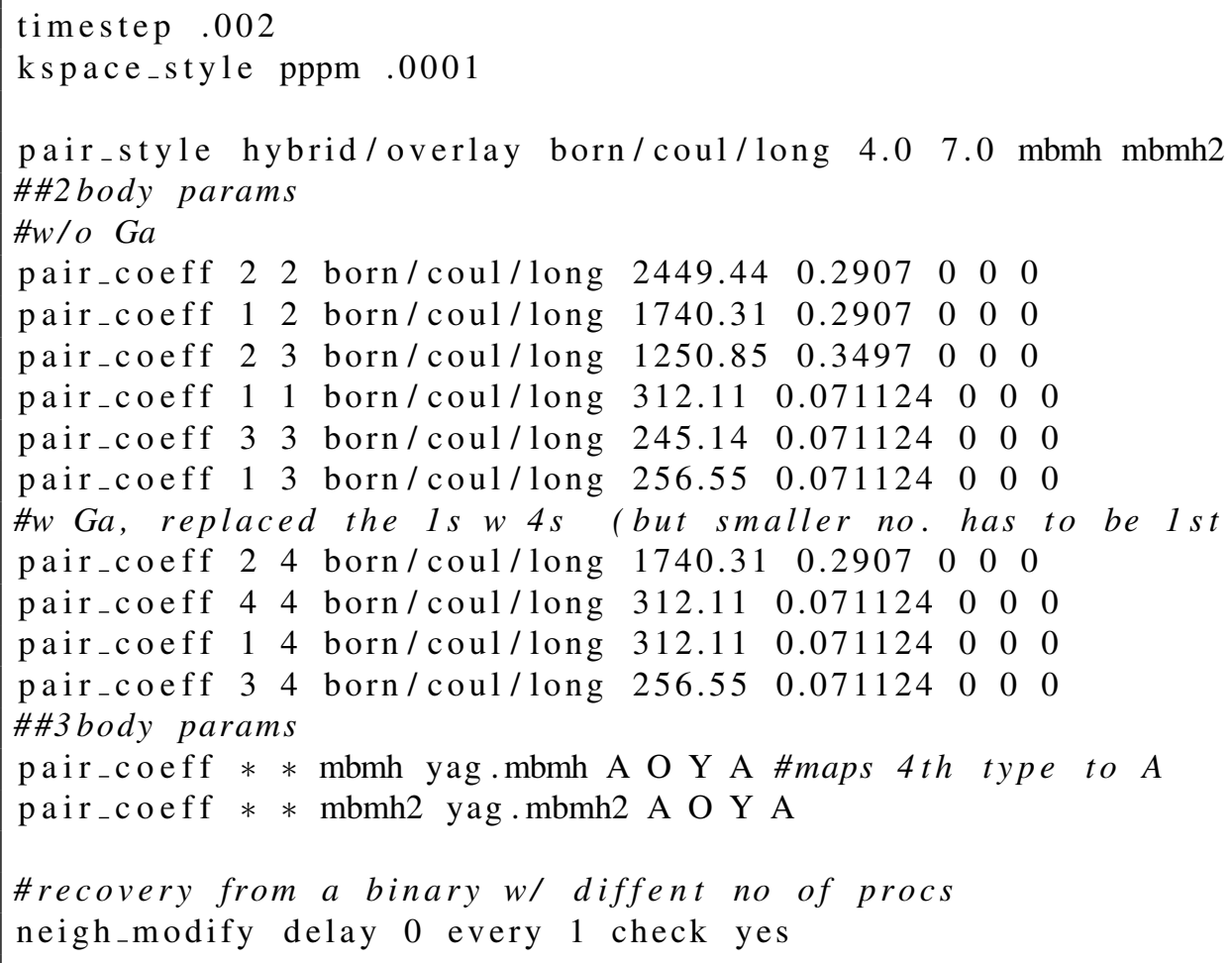

\section{3-Body parameters (yag.mbmh)}

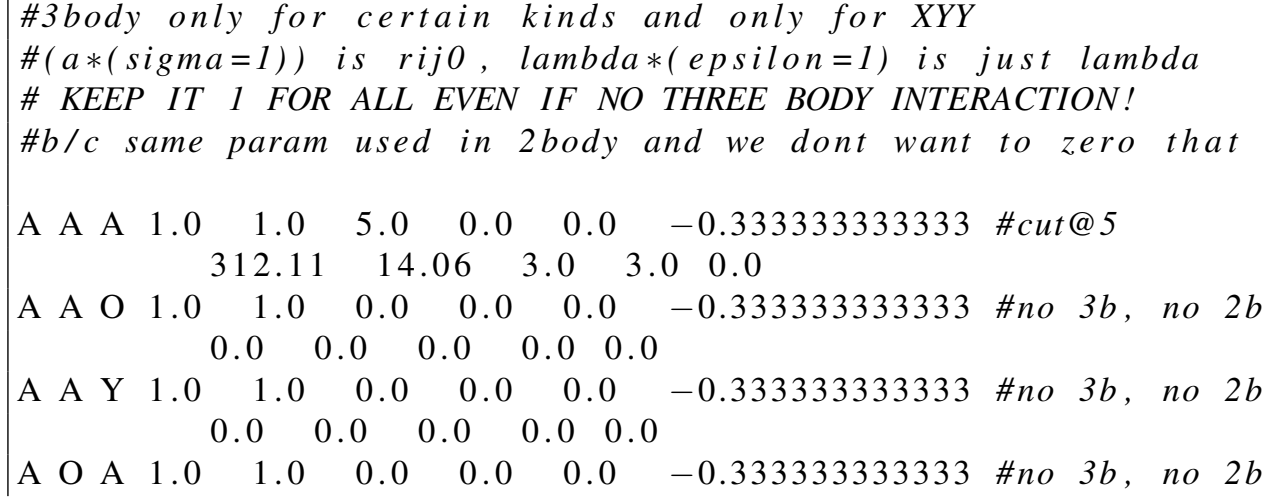




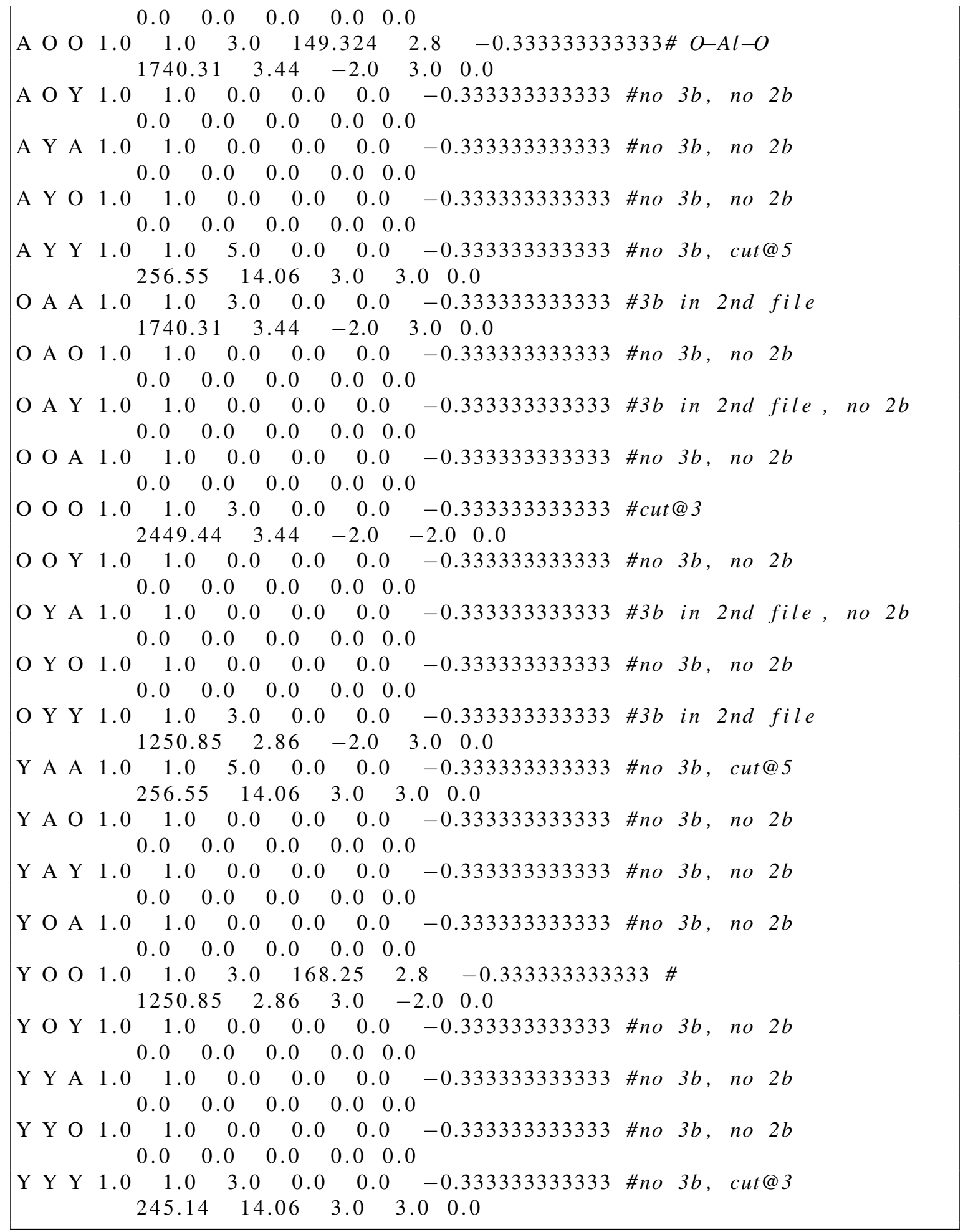

3-Body parameters (yag.mbmh2)

\#3body only for certain kinds and only for XYY

\# $(a *(\operatorname{sigma}=1))$ is rijO, lambda*(epsilon $=1)$ is just lambda

\# KEEP IT I FOR ALL EVEN IF NO THREE BODY INTERACTION!

\#actually zero the two-body interaction here 


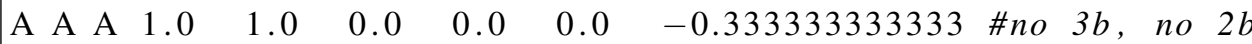
$\begin{array}{lllll}0.0 & 0.0 & 0.0 & 0.0 & 0.0\end{array}$

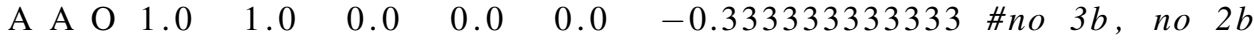
$\begin{array}{lllll}0.0 & 0.0 & 0.0 & 0.0 & 0.0\end{array}$

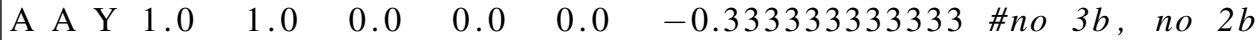
$\begin{array}{lllll}0.0 & 0.0 & 0.0 & 0.0 & 0.0\end{array}$

$\begin{array}{lllllllll}\text { A O A } 1.0 & 1.0 & 0.0 & 0.0 & 0.0 & -0.333333333333\end{array}$ \#no $3 b$, no $2 b$ $\begin{array}{lllll}0.0 & 0.0 & 0.0 & 0.0 & 0.0\end{array}$

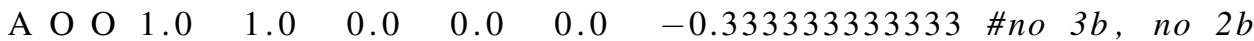
$\begin{array}{lllll}0.0 & 0.0 & 0.0 & 0.0 & 0.0\end{array}$

$\begin{array}{llllllllll}\text { A O Y } & 1.0 & 1.0 & 0.0 & 0.0 & 0.0 & -0.333333333333\end{array}$ \#no $3 b$, no $2 b$ $\begin{array}{lllll}0.0 & 0.0 & 0.0 & 0.0 & 0.0\end{array}$

A Y A $1.0 \quad 1.0 \quad 0.0 \quad 0.0 \quad 0.0 \quad-0.333333333333$ \#no $3 b$, no $2 b$ $\begin{array}{lllll}0.0 & 0.0 & 0.0 & 0.0 & 0.0\end{array}$

$\begin{array}{llllllllll}\text { A } & Y & O & 1.0 & 1.0 & 0.0 & 0.0 & 0.0 & -0.333333333333\end{array}$ \#no $3 b$, no $2 b$ $\begin{array}{lllll}0.0 & 0.0 & 0.0 & 0.0 & 0.0\end{array}$

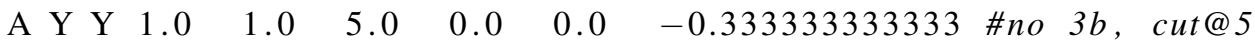
$\begin{array}{lllll}256.55 & 14.06 & 3.0 & 3.0 & 0.0\end{array}$

$\begin{array}{lllllllll}\mathrm{O} & \mathrm{A} & \mathrm{A} & 1.0 & 1.0 & 2.6 & 6.242 & 2.0 & -0.333333333333\end{array}$ \#3b in this file $\begin{array}{lllll}0.0 & 0.0 & 0.0 & 0.0 & 0.0\end{array}$

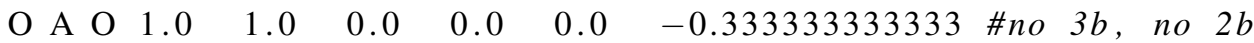
$\begin{array}{lllll}0.0 & 0.0 & 0.0 & 0.0 & 0.0\end{array}$

$\mathrm{O}$ A Y $1.0 \quad 1.0 \quad 2.6 \quad 6.242 \quad 2.0 \quad-0.333333333333$ \#3b in this file (symm ?) , no $2 b$

$\begin{array}{lllll}0.0 & 0.0 & 0.0 & 0.0 & 0.0\end{array}$

O O A $1.0 \quad 1.0 \quad 0.0 \quad 0.0 \quad 0.0 \quad-0.333333333333$ \#no $3 b$, no $2 b$ $\begin{array}{lllll}0.0 & 0.0 & 0.0 & 0.0 & 0.0\end{array}$

$\begin{array}{llllllllll}\mathrm{O} & \mathrm{O} & \mathrm{O} & 1.0 & 1.0 & 0.0 & 0.0 & 0.0 & -0.333333333333\end{array}$ \#no $3 b$, no $2 b$ $\begin{array}{lllll}0.0 & 0.0 & 0.0 & 0.0 & 0.0\end{array}$

$\begin{array}{lllllllll}\text { O O Y } & 1.0 & 1.0 & 0.0 & 0.0 & 0.0 & -0.333333333333 & \text { \#no } 3 b, \text { no } 2 b\end{array}$ $\begin{array}{lllll}0.0 & 0.0 & 0.0 & 0.0 & 0.0\end{array}$

$\mathrm{O}$ Y A $1.0 \quad 1.0 \quad 2.6 \quad 6.242 \quad 2.0 \quad-0.333333333333$ \#3b in this file (symm ?) , no $2 b$ $\begin{array}{lllll}0.0 & 0.0 & 0.0 & 0.0 & 0.0\end{array}$

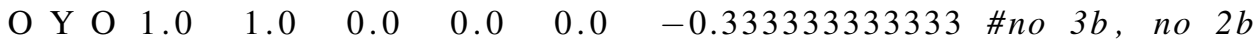
$\begin{array}{lllll}0.0 & 0.0 & 0.0 & 0.0 & 0.0\end{array}$

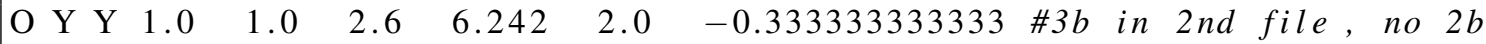
$\begin{array}{lllll}0.0 & 0.0 & 0.0 & 0.0 & 0.0\end{array}$

Y A A $1.0 \quad 1.0 \quad 0.0 \quad 0.0 \quad 0.0 \quad-0.333333333333$ \#no $3 b$, no $2 b$ $\begin{array}{lllll}0.0 & 0.0 & 0.0 & 0.0 & 0.0\end{array}$

Y A O $1.0 \quad 1.0 \quad 0.0 \quad 0.0 \quad 0.0 \quad-0.333333333333$ \#no $3 b$, no $2 b$ $\begin{array}{lllll}0.0 & 0.0 & 0.0 & 0.0 & 0.0\end{array}$

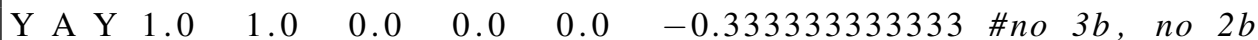
$\begin{array}{lllll}0.0 & 0.0 & 0.0 & 0.0 & 0.0\end{array}$

Y O A $1.0 \quad 1.0 \quad 0.0 \quad 0.0 \quad 0.0 \quad-0.333333333333$ \#no $3 b$, no $2 b$ $\begin{array}{lllll}0.0 & 0.0 & 0.0 & 0.0 & 0.0\end{array}$

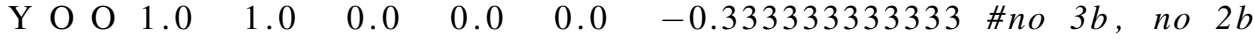
$\begin{array}{lllll}0.0 & 0.0 & 0.0 & 0.0 & 0.0\end{array}$

Y O Y $1.0 \quad 1.0 \quad 0.0 \quad 0.0 \quad 0.0 \quad-0.333333333333$ \#no $3 b$, no $2 b$ $\begin{array}{lllll}0.0 & 0.0 & 0.0 & 0.0 & 0.0\end{array}$

Y Y A $1.0 \quad 1.0 \quad 0.0 \quad 0.0 \quad 0.0 \quad-0.333333333333$ \#no $3 b$, no $2 b$ $\begin{array}{lllll}0.0 & 0.0 & 0.0 & 0.0 & 0.0\end{array}$

$\begin{array}{llllllllll}\text { Y Y O } & 1.0 & 1.0 & 0.0 & 0.0 & 0.0 & -0.333333333333 & \text { \#no } & 3 b, \text { no } 2 b\end{array}$ 


$\begin{array}{ccccccccc} & & 0.0 & 0.0 & 0.0 & 0.0 & 0.0 & & \\ \text { Y Y Y } 1.0 & 1.0 & 0.0 & 0.0 & 0.0 & -0.333333333333 & \text { \#no } 3 b, \text { no } 2 b \\ & 0.0 & 0.0 & 0.0 & 0.0 & 0.0\end{array}$

\section{Simulation Execution}

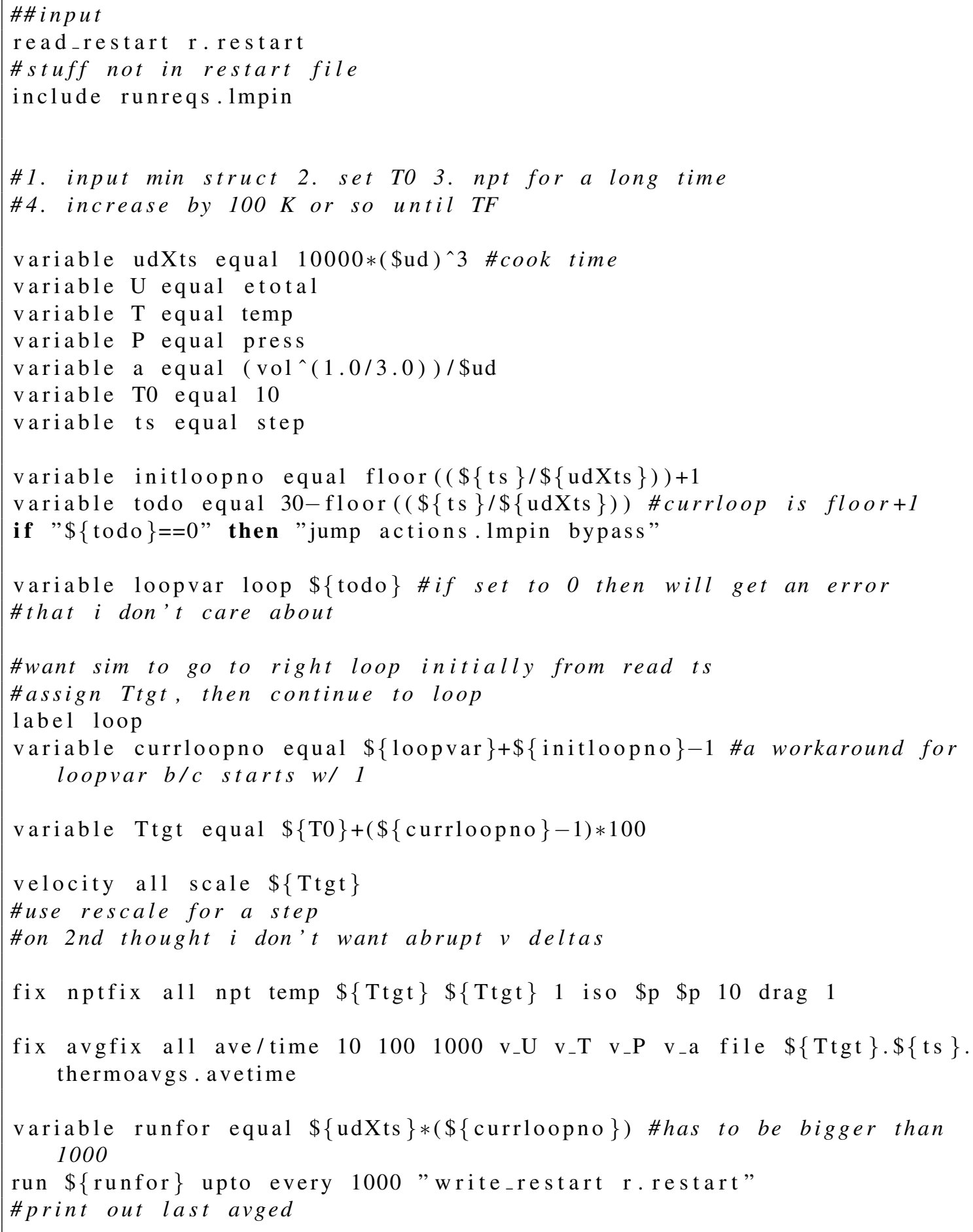


next loopvar

jump actions.lmpin loop

label bypass

LAMMPS SCRIPTS FOR MELTING TEMPERATURE

\section{Simulation Setup}

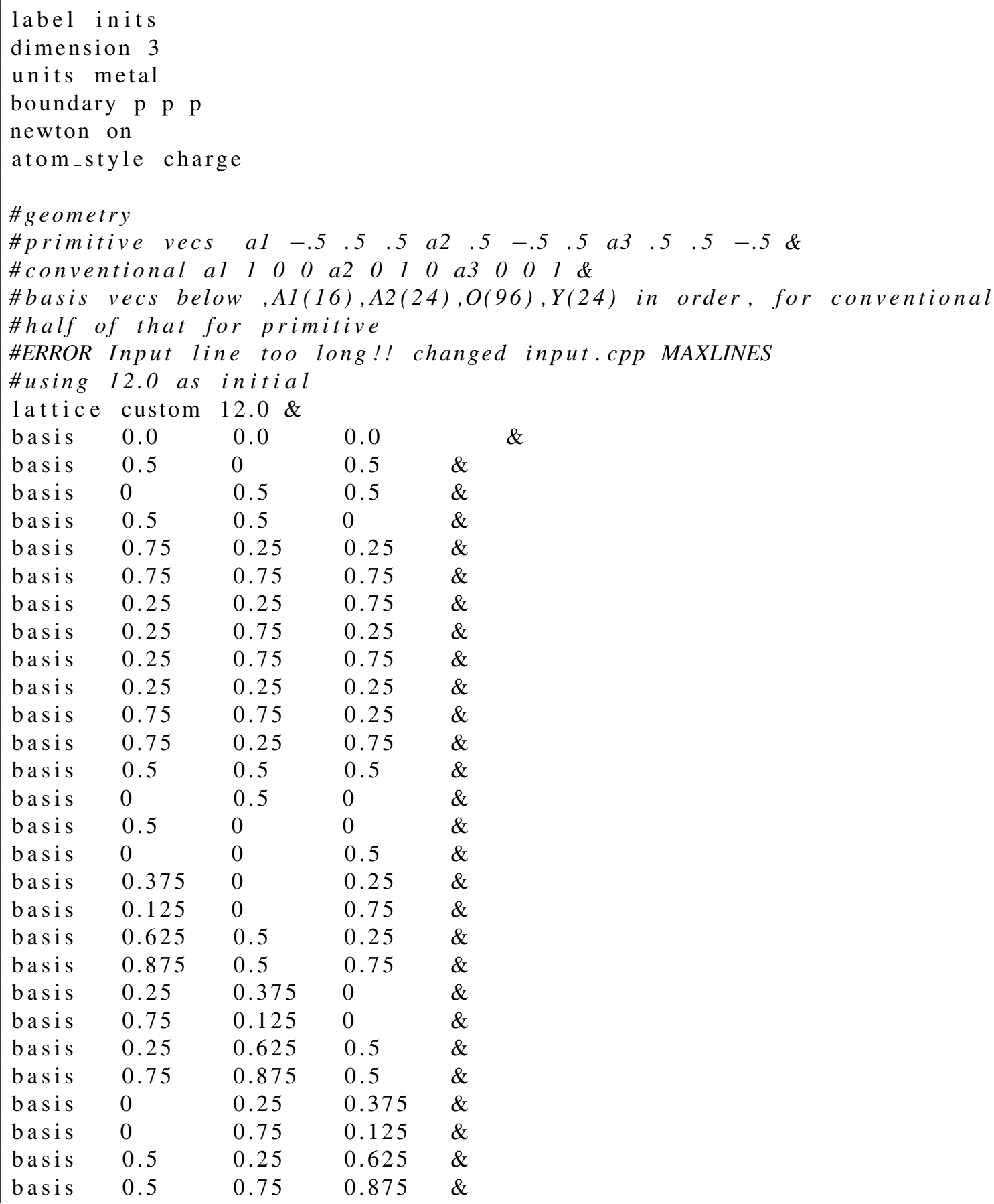




\begin{tabular}{|lllll} 
basis & 0.75 & 0.625 & 0 & $\&$ \\
basis & 0.75 & 0.375 & 0.5 & $\&$ \\
basis & 0.25 & 0.875 & 0 & $\&$ \\
basis & 0.25 & 0.125 & 0.5 & $\&$ \\
basis & 0.125 & 0.5 & 0.25 & $\&$ \\
basis & 0.875 & 0 & 0.25 & $\&$ \\
basis & 0.375 & 0.5 & 0.75 & $\&$ \\
basis & 0.625 & 0 & 0.75 & $\&$ \\
basis & 0 & 0.25 & 0.875 & $\&$ \\
basis & 0.5 & 0.25 & 0.125 & $\&$ \\
basis & 0 & 0.75 & 0.625 & $\&$ \\
basis & 0.5 & 0.75 & 0.375 & $\&$ \\
basis & 0.28023 & 0.1011 & 0.19922 & $\&$ \\
basis & 0.21977 & 0.8989 & 0.69922 & $\&$ \\
basis & 0.71977 & 0.6011 & 0.30078 & $\&$ \\
basis & 0.78023 & 0.3989 & 0.80078 & $\&$ \\
basis & 0.19922 & 0.28023 & 0.1011 & $\&$ \\
basis & 0.69922 & 0.21977 & 0.8989 & $\&$ \\
basis & 0.30078 & 0.71977 & 0.6011 & $\&$ \\
basis & 0.80078 & 0.78023 & 0.3989 & $\&$ \\
basis & 0.1011 & 0.19922 & 0.28023 & $\&$ \\
basis & 0.8989 & 0.69922 & 0.21977 & $\&$ \\
basis & 0.6011 & 0.30078 & 0.71977 & $\&$ \\
basis & 0.3989 & 0.80078 & 0.78023 & $\&$ \\
basis & 0.8511 & 0.53023 & 0.05078 & $\&$ \\
basis & 0.6489 & 0.46977 & 0.55078 & $\&$ \\
basis & 0.3511 & 0.96977 & 0.94922 & $\&$ \\
basis & 0.1489 & 0.03023 & 0.44922 & $\&$ \\
basis & 0.03023 & 0.44922 & 0.1489 & $\&$ \\
basis & 0.96977 & 0.94922 & 0.3511 & $\&$ \\
basis & 0.46977 & 0.55078 & 0.6489 & $\&$ \\
basis & 0.53023 & 0.05078 & 0.8511 & $\&$ \\
basis & 0.94922 & 0.3511 & 0.96977 & $\&$ \\
basis & 0.44922 & 0.1489 & 0.03023 & $\&$ \\
basis & 0.05078 & 0.8511 & 0.53023 & $\&$ \\
basis & 0.55078 & 0.6489 & 0.46977 & $\&$ \\
basis & 0.71977 & 0.8989 & 0.80078 & $\&$ \\
basis & 0.78023 & 0.1011 & 0.30078 & $\&$ \\
basis & 0.28023 & 0.3989 & 0.69922 & $\&$ \\
basis & 0.21977 & 0.6011 & 0.19922 & $\&$ \\
basis & 0.80078 & 0.71977 & 0.8989 & $\&$ \\
basis & 0.30078 & 0.78023 & 0.1011 & $\&$ \\
basis & 0.69922 & 0.28023 & 0.3989 & $\&$ \\
basis & 0.19922 & 0.21977 & 0.6011 & $\&$ \\
basis & 0.8989 & 0.80078 & 0.71977 & $\&$ \\
basis & 0.1011 & 0.30078 & 0.78023 & $\&$ \\
basis & 0.3989 & 0.69922 & 0.28023 & $\&$ \\
basis & 0.6011 & 0.19922 & 0.21977 & $\&$ \\
basis & 0.1489 & 0.46977 & 0.94922 & $\&$ \\
basis & 0.3511 & 0.53023 & 0.44922 & $\&$ \\
basis & 0.6489 & 0.03023 & 0.05078 & $\&$ \\
basis & 0.8511 & 0.96977 & 0.55078 & $\&$ \\
basis & 0.96977 & 0.55078 & 0.8511 & $\&$ \\
basis & 0.03023 & 0.05078 & 0.6489 & $\&$
\end{tabular}




\begin{tabular}{|lllll} 
basis & 0.53023 & 0.44922 & 0.3511 & $\&$ \\
basis & 0.46977 & 0.94922 & 0.1489 & $\&$ \\
basis & 0.05078 & 0.6489 & 0.03023 & $\&$ \\
basis & 0.55078 & 0.8511 & 0.96977 & $\&$ \\
basis & 0.94922 & 0.1489 & 0.46977 & $\&$ \\
basis & 0.44922 & 0.3511 & 0.53023 & $\&$ \\
basis & 0.78023 & 0.6011 & 0.69922 & $\&$ \\
basis & 0.71977 & 0.3989 & 0.19922 & $\&$ \\
basis & 0.21977 & 0.1011 & 0.80078 & $\&$ \\
basis & 0.28023 & 0.8989 & 0.30078 & $\&$ \\
basis & 0.69922 & 0.78023 & 0.6011 & $\&$ \\
basis & 0.19922 & 0.71977 & 0.3989 & $\&$ \\
basis & 0.80078 & 0.21977 & 0.1011 & $\&$ \\
basis & 0.30078 & 0.28023 & 0.8989 & $\&$ \\
basis & 0.6011 & 0.69922 & 0.78023 & $\&$ \\
basis & 0.3989 & 0.19922 & 0.71977 & $\&$ \\
basis & 0.1011 & 0.80078 & 0.21977 & $\&$ \\
basis & 0.8989 & 0.30078 & 0.28023 & $\&$ \\
basis & 0.3511 & 0.03023 & 0.55078 & $\&$ \\
basis & 0.1489 & 0.96977 & 0.05078 & $\&$ \\
basis & 0.8511 & 0.46977 & 0.44922 & $\&$ \\
basis & 0.6489 & 0.53023 & 0.94922 & $\&$ \\
basis & 0.53023 & 0.94922 & 0.6489 & $\&$ \\
basis & 0.46977 & 0.44922 & 0.8511 & $\&$ \\
basis & 0.96977 & 0.05078 & 0.1489 & $\&$ \\
basis & 0.03023 & 0.55078 & 0.3511 & $\&$ \\
basis & 0.44922 & 0.8511 & 0.46977 & $\&$ \\
basis & 0.94922 & 0.6489 & 0.53023 & $\&$ \\
basis & 0.55078 & 0.3511 & 0.03023 & $\&$ \\
basis & 0.05078 & 0.1489 & 0.96977 & $\&$ \\
basis & 0.21977 & 0.3989 & 0.30078 & $\&$ \\
basis & 0.28023 & 0.6011 & 0.80078 & $\&$ \\
basis & 0.78023 & 0.8989 & 0.19922 & $\&$ \\
basis & 0.71977 & 0.1011 & 0.69922 & $\&$ \\
basis & 0.30078 & 0.21977 & 0.3989 & $\&$ \\
basis & 0.80078 & 0.28023 & 0.6011 & $\&$ \\
basis & 0.19922 & 0.78023 & 0.8989 & $\&$ \\
basis & 0.69922 & 0.71977 & 0.1011 & $\&$ \\
basis & 0.3989 & 0.30078 & 0.21977 & $\&$ \\
basis & 0.6011 & 0.80078 & 0.28023 & $\&$ \\
basis & 0.8989 & 0.19922 & 0.78023 & $\&$ \\
basis & 0.1011 & 0.69922 & 0.71977 & $\&$ \\
basis & 0.6489 & 0.96977 & 0.44922 & $\&$ \\
basis & 0.8511 & 0.03023 & 0.94922 & $\&$ \\
basis & 0.1489 & 0.53023 & 0.55078 & $\&$ \\
basis & 0.3511 & 0.46977 & 0.05078 & $\&$ \\
basis & 0.46977 & 0.05078 & 0.3511 & $\&$ \\
basis & 0.53023 & 0.55078 & 0.1489 & $\&$ \\
basis & 0.03023 & 0.94922 & 0.8511 & $\&$ \\
basis & 0.96977 & 0.44922 & 0.6489 & $\&$ \\
basis & 0.55078 & 0.1489 & 0.53023 & $\&$ \\
basis & 0.05078 & 0.3511 & 0.46977 & $\&$ \\
basis & 0.44922 & 0.6489 & 0.96977 & $\&$ \\
basis & 0.94922 & 0.8511 & 0.03023 & $\&$
\end{tabular}




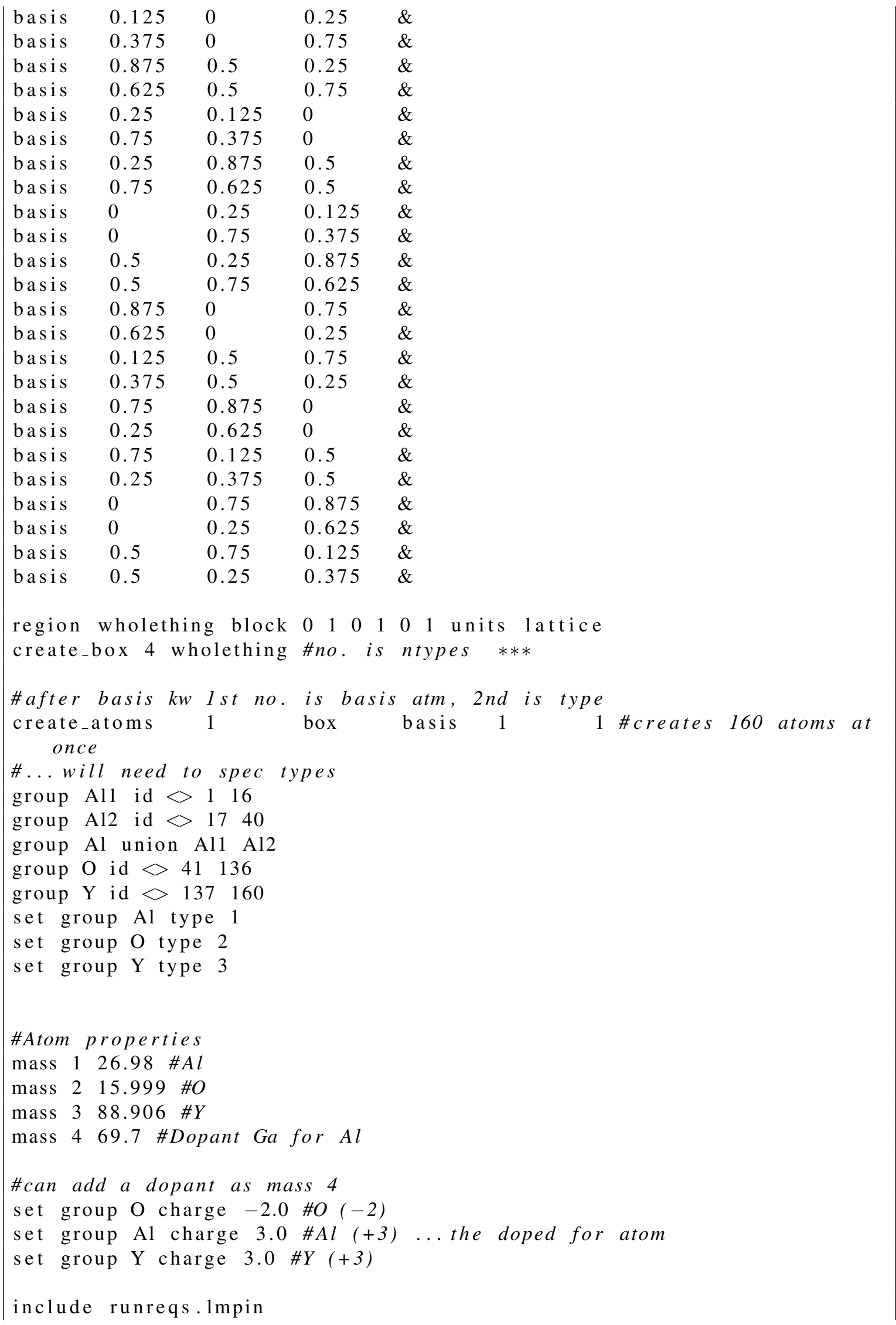




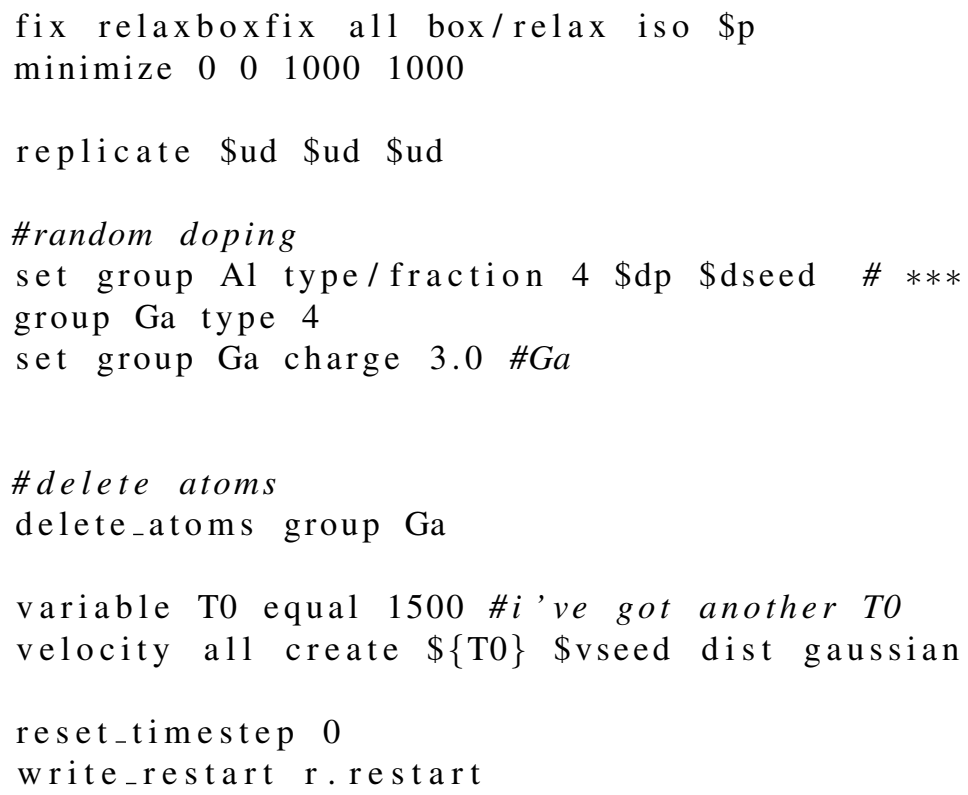

\section{Interatomic Potentials}

runreqs.lmpin

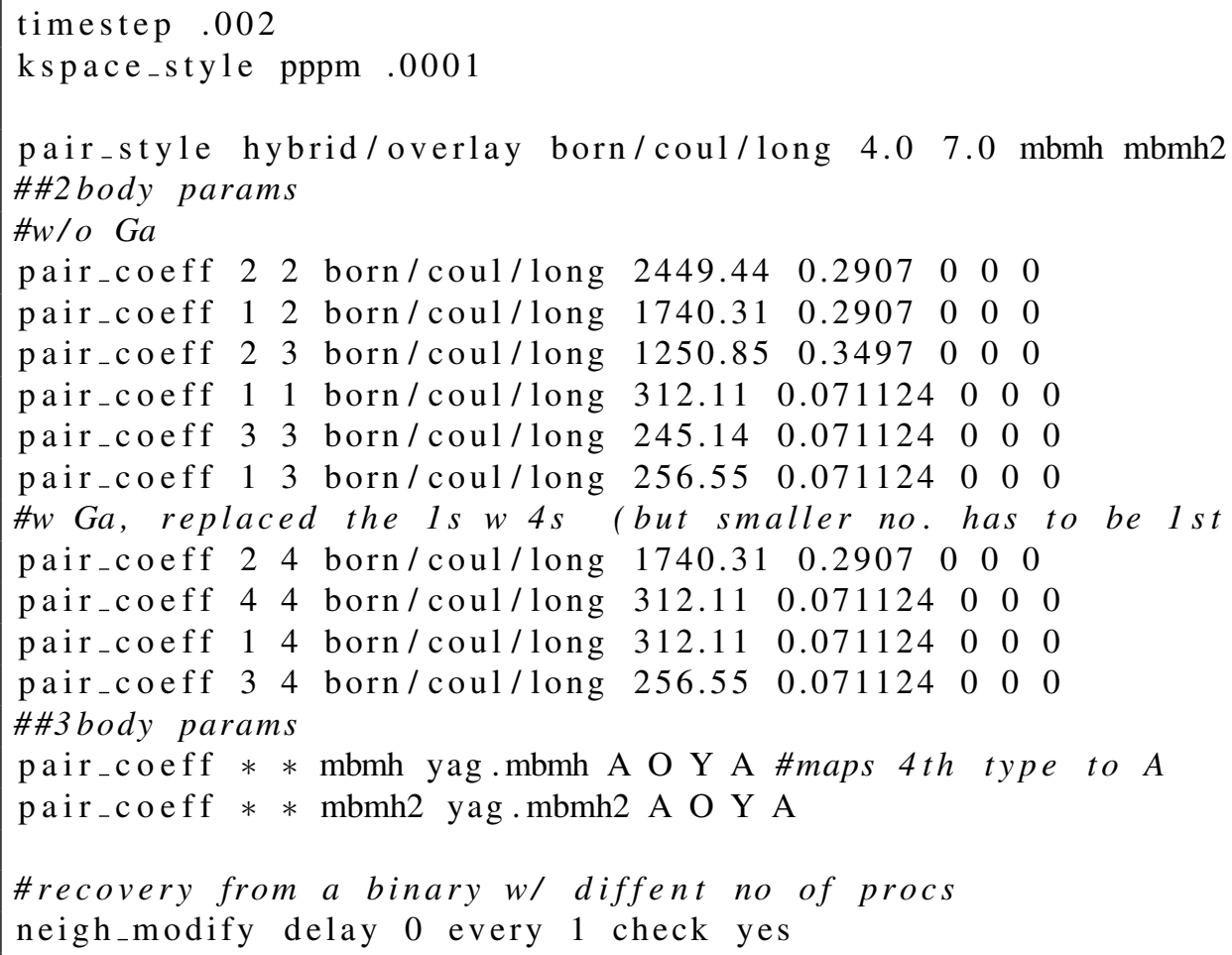

3-Body parameters (yag.mbmh)

\#3body only for certain kinds and only for XYY

$\#(a *(\operatorname{sigma}=1))$ is rijO, lambda*(epsilon=1) is just lambda

\# KEEP IT 1 FOR ALL EVEN IF NO THREE BODY INTERACTION! 


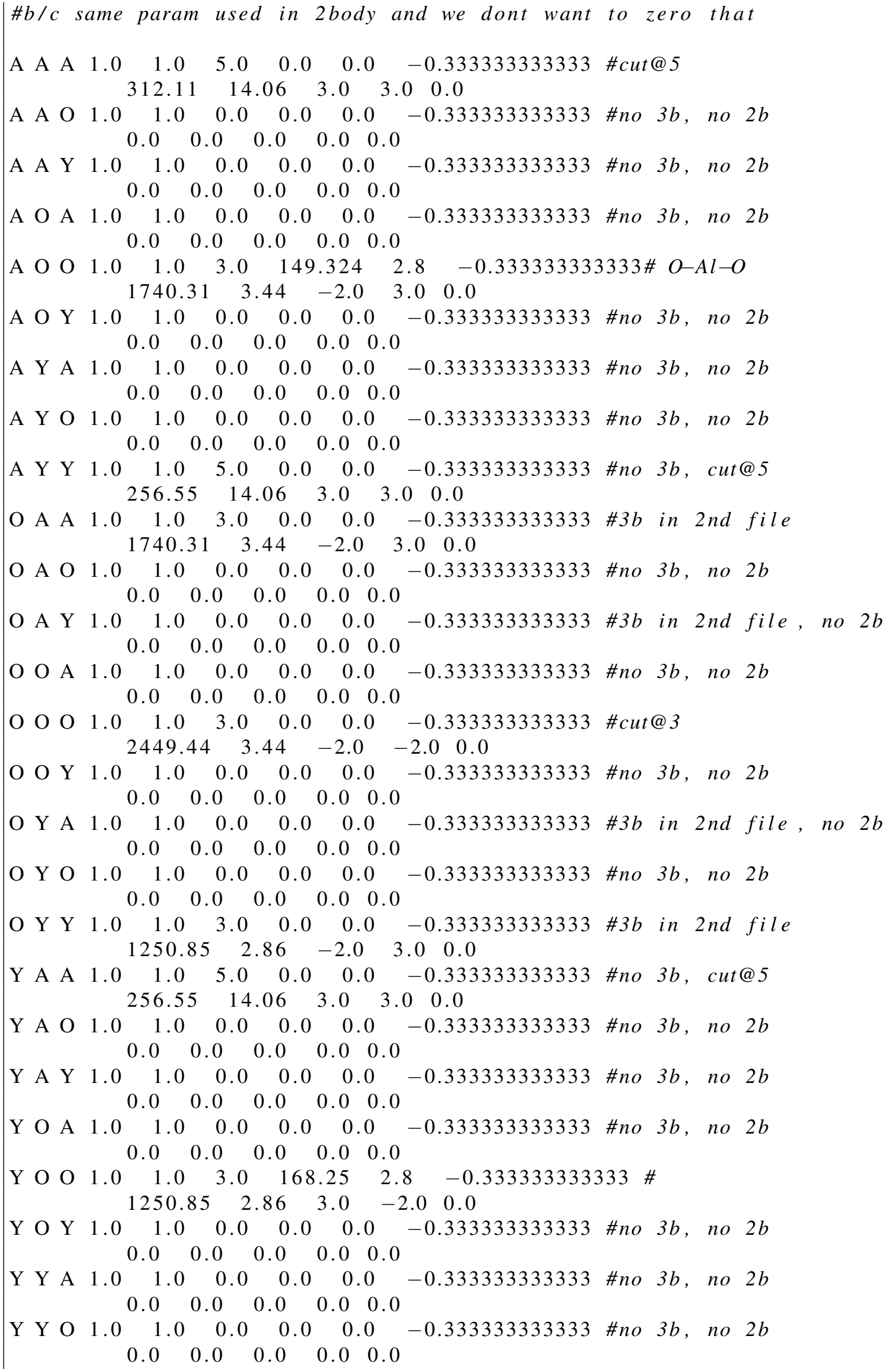


\begin{tabular}{|llllllllll} 
Y Y Y & 1.0 & 1.0 & 3.0 & 0.0 & 0.0 & -0.333333333333 & \#no & 3b, cut@3
\end{tabular} $245.14 \quad 14.06 \quad 3.0 \quad 3.0 \quad 0.0$

3-Body parameters (yag.mbmh2)

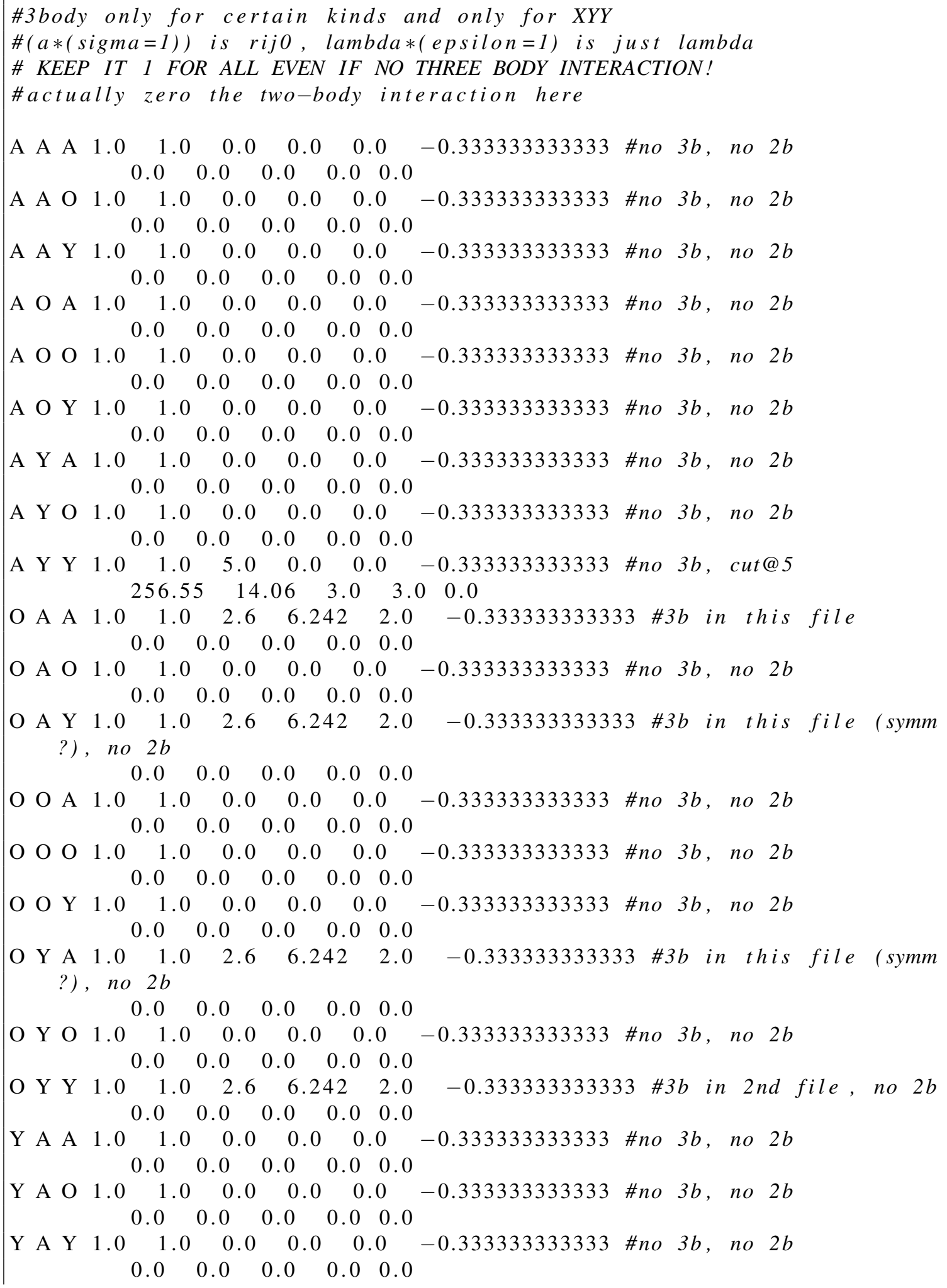




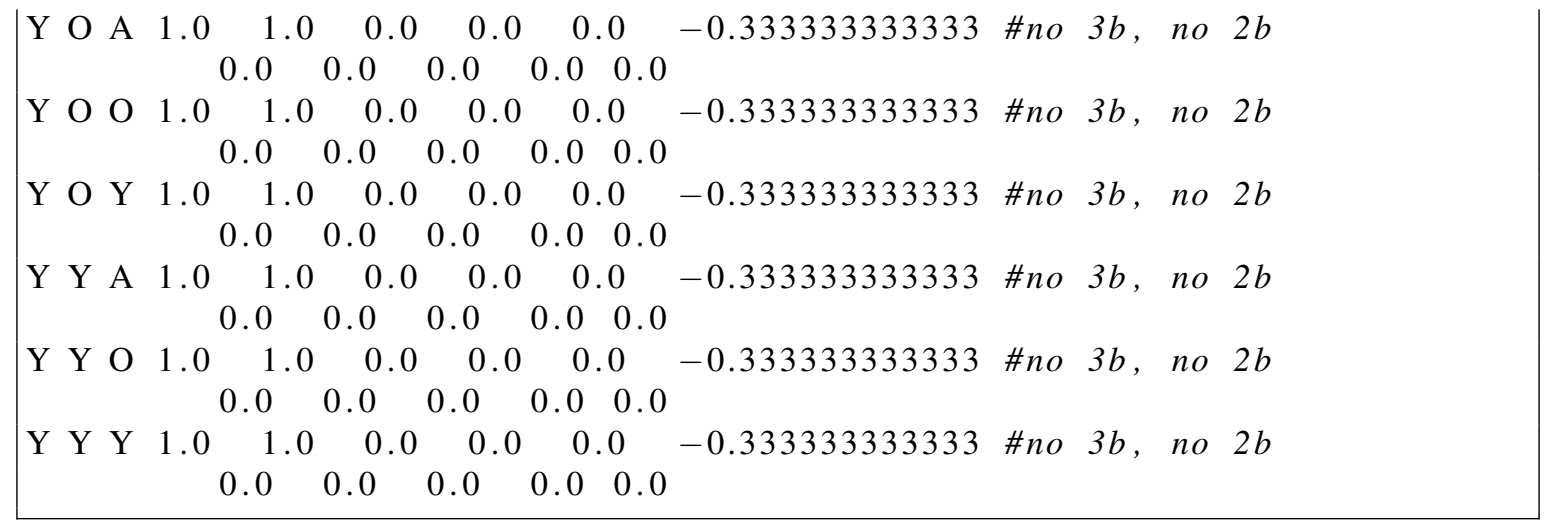

\section{Simulation Execution}

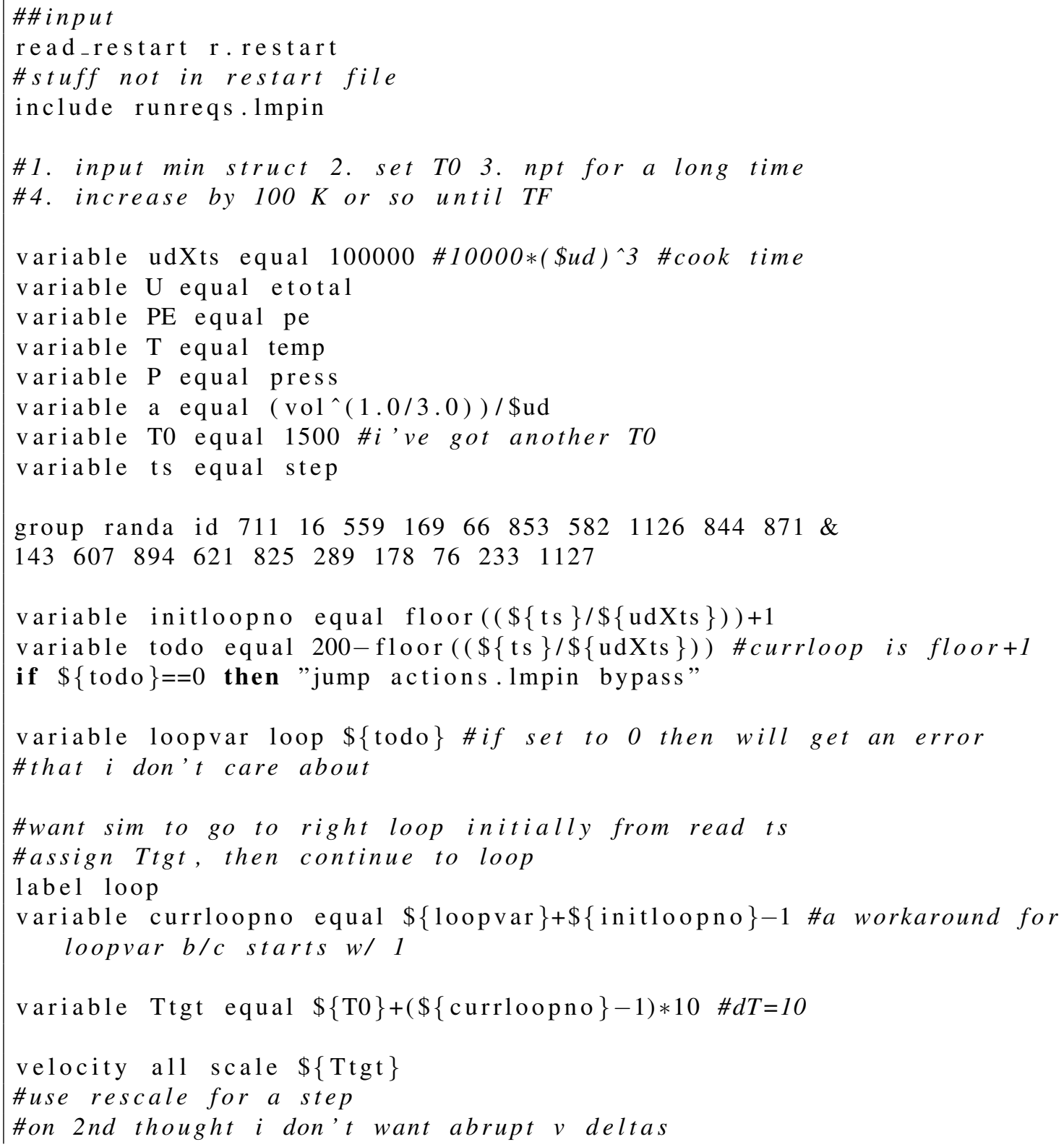




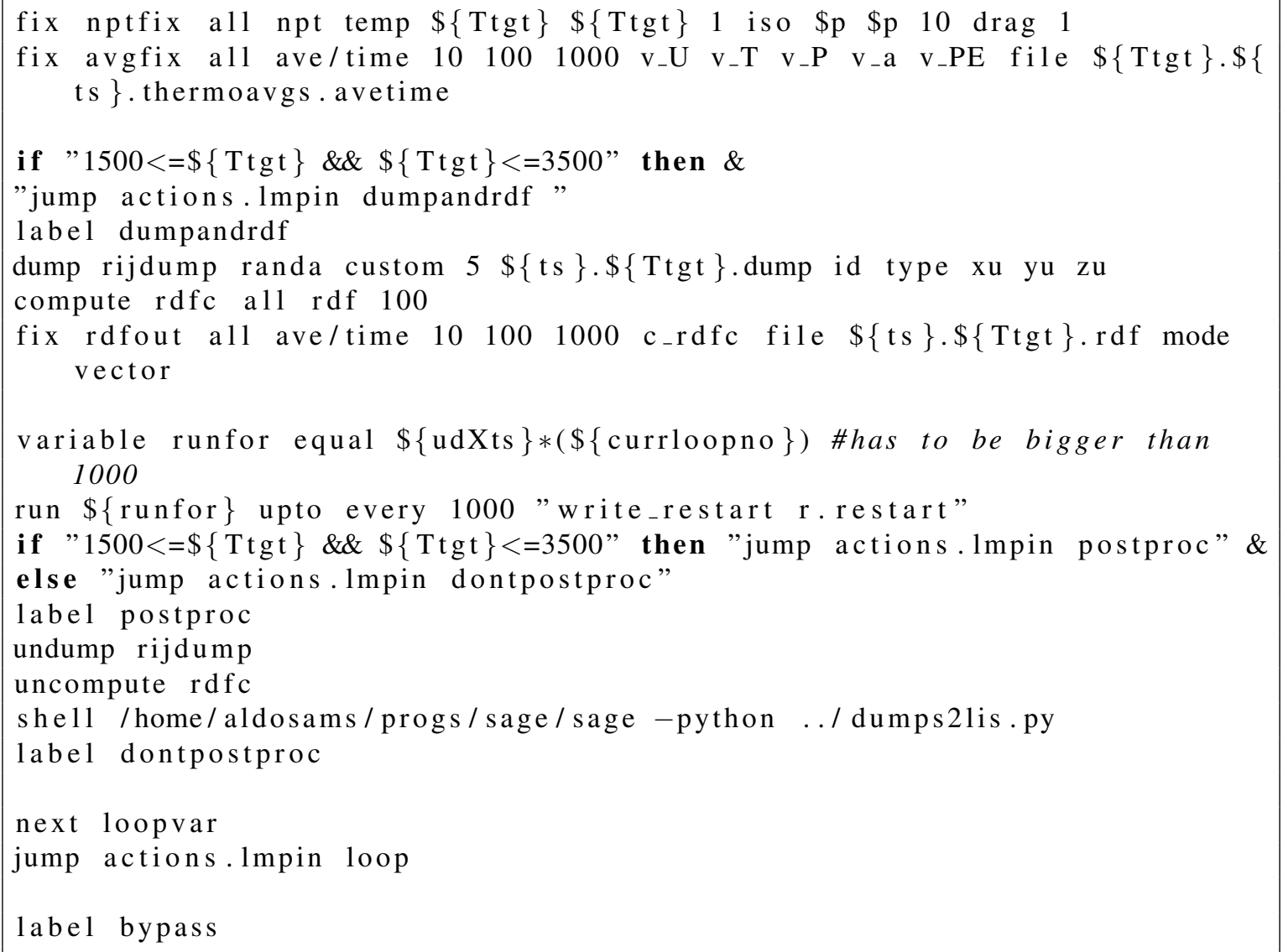




\section{LAMMPS NON-EQUILIBRIUM THERMAL CONDUCTIVITY CODE Simulation Setup Script}

This script sets up the simulation.

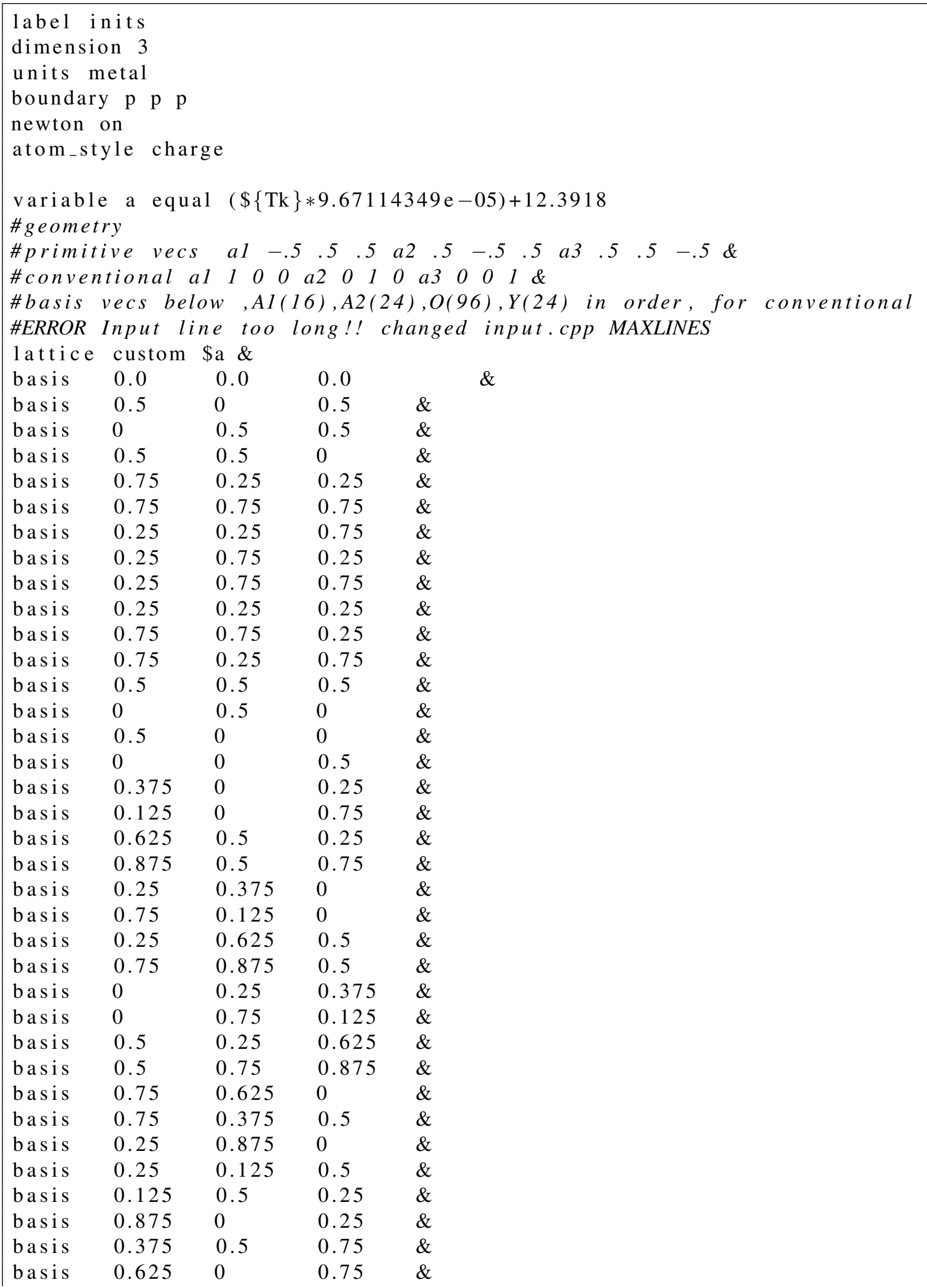




\begin{tabular}{|lllll} 
basis & 0 & 0.25 & 0.875 & $\&$ \\
basis & 0.5 & 0.25 & 0.125 & $\&$ \\
basis & 0 & 0.75 & 0.625 & $\&$ \\
basis & 0.5 & 0.75 & 0.375 & $\&$ \\
basis & 0.28023 & 0.1011 & 0.19922 & $\&$ \\
basis & 0.21977 & 0.8989 & 0.69922 & $\&$ \\
basis & 0.71977 & 0.6011 & 0.30078 & $\&$ \\
basis & 0.78023 & 0.3989 & 0.80078 & $\&$ \\
basis & 0.19922 & 0.28023 & 0.1011 & $\&$ \\
basis & 0.69922 & 0.21977 & 0.8989 & $\&$ \\
basis & 0.30078 & 0.71977 & 0.6011 & $\&$ \\
basis & 0.80078 & 0.78023 & 0.3989 & $\&$ \\
basis & 0.1011 & 0.19922 & 0.28023 & $\&$ \\
basis & 0.8989 & 0.69922 & 0.21977 & $\&$ \\
basis & 0.6011 & 0.30078 & 0.71977 & $\&$ \\
basis & 0.3989 & 0.80078 & 0.78023 & $\&$ \\
basis & 0.8511 & 0.53023 & 0.05078 & $\&$ \\
basis & 0.6489 & 0.46977 & 0.55078 & $\&$ \\
basis & 0.3511 & 0.96977 & 0.94922 & $\&$ \\
basis & 0.1489 & 0.03023 & 0.44922 & $\&$ \\
basis & 0.03023 & 0.44922 & 0.1489 & $\&$ \\
basis & 0.96977 & 0.94922 & 0.3511 & $\&$ \\
basis & 0.46977 & 0.55078 & 0.6489 & $\&$ \\
basis & 0.53023 & 0.05078 & 0.8511 & $\&$ \\
basis & 0.94922 & 0.3511 & 0.96977 & $\&$ \\
basis & 0.44922 & 0.1489 & 0.03023 & $\&$ \\
basis & 0.05078 & 0.8511 & 0.53023 & $\&$ \\
basis & 0.55078 & 0.6489 & 0.46977 & $\&$ \\
basis & 0.71977 & 0.8989 & 0.80078 & $\&$ \\
basis & 0.78023 & 0.1011 & 0.30078 & $\&$ \\
basis & 0.28023 & 0.3989 & 0.69922 & $\&$ \\
basis & 0.21977 & 0.6011 & 0.19922 & $\&$ \\
basis & 0.80078 & 0.71977 & 0.8989 & $\&$ \\
basis & 0.30078 & 0.78023 & 0.1011 & $\&$ \\
basis & 0.69922 & 0.28023 & 0.3989 & $\&$ \\
basis & 0.19922 & 0.21977 & 0.6011 & $\&$ \\
basis & 0.8989 & 0.80078 & 0.71977 & $\&$ \\
basis & 0.1011 & 0.30078 & 0.78023 & $\&$ \\
basis & 0.3989 & 0.69922 & 0.28023 & $\&$ \\
basis & 0.6011 & 0.19922 & 0.21977 & $\&$ \\
basis & 0.1489 & 0.46977 & 0.94922 & $\&$ \\
basis & 0.3511 & 0.53023 & 0.44922 & $\&$ \\
basis & 0.6489 & 0.03023 & 0.05078 & $\&$ \\
basis & 0.8511 & 0.96977 & 0.55078 & $\&$ \\
basis & 0.96977 & 0.55078 & 0.8511 & $\&$ \\
basis & 0.03023 & 0.05078 & 0.6489 & $\&$ \\
basis & 0.53023 & 0.44922 & 0.3511 & $\&$ \\
basis & 0.46977 & 0.94922 & 0.1489 & $\&$ \\
basis & 0.05078 & 0.6489 & 0.03023 & $\&$ \\
basis & 0.55078 & 0.8511 & 0.96977 & $\&$ \\
basis & 0.94922 & 0.1489 & 0.46977 & $\&$ \\
basis & 0.44922 & 0.3511 & 0.53023 & $\&$ \\
basis & 0.78023 & 0.6011 & 0.69922 & $\&$ \\
basis & 0.71977 & 0.3989 & 0.19922 & $\&$
\end{tabular}




\begin{tabular}{|lllll} 
basis & 0.21977 & 0.1011 & 0.80078 & $\&$ \\
basis & 0.28023 & 0.8989 & 0.30078 & $\&$ \\
basis & 0.69922 & 0.78023 & 0.6011 & $\&$ \\
basis & 0.19922 & 0.71977 & 0.3989 & $\&$ \\
basis & 0.80078 & 0.21977 & 0.1011 & $\&$ \\
basis & 0.30078 & 0.28023 & 0.8989 & $\&$ \\
basis & 0.6011 & 0.69922 & 0.78023 & $\&$ \\
basis & 0.3989 & 0.19922 & 0.71977 & $\&$ \\
basis & 0.1011 & 0.80078 & 0.21977 & $\&$ \\
basis & 0.8989 & 0.30078 & 0.28023 & $\&$ \\
basis & 0.3511 & 0.03023 & 0.55078 & $\&$ \\
basis & 0.1489 & 0.96977 & 0.05078 & $\&$ \\
basis & 0.8511 & 0.46977 & 0.44922 & $\&$ \\
basis & 0.6489 & 0.53023 & 0.94922 & $\&$ \\
basis & 0.53023 & 0.94922 & 0.6489 & $\&$ \\
basis & 0.46977 & 0.44922 & 0.8511 & $\&$ \\
basis & 0.96977 & 0.05078 & 0.1489 & $\&$ \\
basis & 0.03023 & 0.55078 & 0.3511 & $\&$ \\
basis & 0.44922 & 0.8511 & 0.46977 & $\&$ \\
basis & 0.94922 & 0.6489 & 0.53023 & $\&$ \\
basis & 0.55078 & 0.3511 & 0.03023 & $\&$ \\
basis & 0.05078 & 0.1489 & 0.96977 & $\&$ \\
basis & 0.21977 & 0.3989 & 0.30078 & $\&$ \\
basis & 0.28023 & 0.6011 & 0.80078 & $\&$ \\
basis & 0.78023 & 0.8989 & 0.19922 & $\&$ \\
basis & 0.71977 & 0.1011 & 0.69922 & $\&$ \\
basis & 0.30078 & 0.21977 & 0.3989 & $\&$ \\
basis & 0.80078 & 0.28023 & 0.6011 & $\&$ \\
basis & 0.19922 & 0.78023 & 0.8989 & $\&$ \\
basis & 0.69922 & 0.71977 & 0.1011 & $\&$ \\
basis & 0.3989 & 0.30078 & 0.21977 & $\&$ \\
basis & 0.6011 & 0.80078 & 0.28023 & $\&$ \\
basis & 0.8989 & 0.19922 & 0.78023 & $\&$ \\
basis & 0.1011 & 0.69922 & 0.71977 & $\&$ \\
basis & 0.6489 & 0.96977 & 0.44922 & $\&$ \\
basis & 0.8511 & 0.03023 & 0.94922 & $\&$ \\
basis & 0.1489 & 0.53023 & 0.55078 & $\&$ \\
basis & 0.3511 & 0.46977 & 0.05078 & $\&$ \\
basis & 0.46977 & 0.05078 & 0.3511 & $\&$ \\
basis & 0.53023 & 0.55078 & 0.1489 & $\&$ \\
basis & 0.03023 & 0.94922 & 0.8511 & $\&$ \\
basis & 0.96977 & 0.44922 & 0.6489 & $\&$ \\
basis & 0.55078 & 0.1489 & 0.53023 & $\&$ \\
basis & 0.05078 & 0.3511 & 0.46977 & $\&$ \\
basis & 0.44922 & 0.6489 & 0.96977 & $\&$ \\
basis & 0.94922 & 0.8511 & 0.03023 & $\&$ \\
basis & 0.125 & 0 & 0.25 & $\&$ \\
basis & 0.375 & 0 & 0.75 & $\&$ \\
basis & 0.875 & 0.5 & 0.25 & $\&$ \\
basis & 0.625 & 0.5 & 0.75 & $\&$ \\
basis & 0.25 & 0.125 & 0 & $\&$ \\
basis & 0.75 & 0.375 & 0 & $\&$ \\
basis & 0.25 & 0.875 & 0.5 & $\&$ \\
basis & 0.75 & 0.625 & 0.5 & $\&$
\end{tabular}




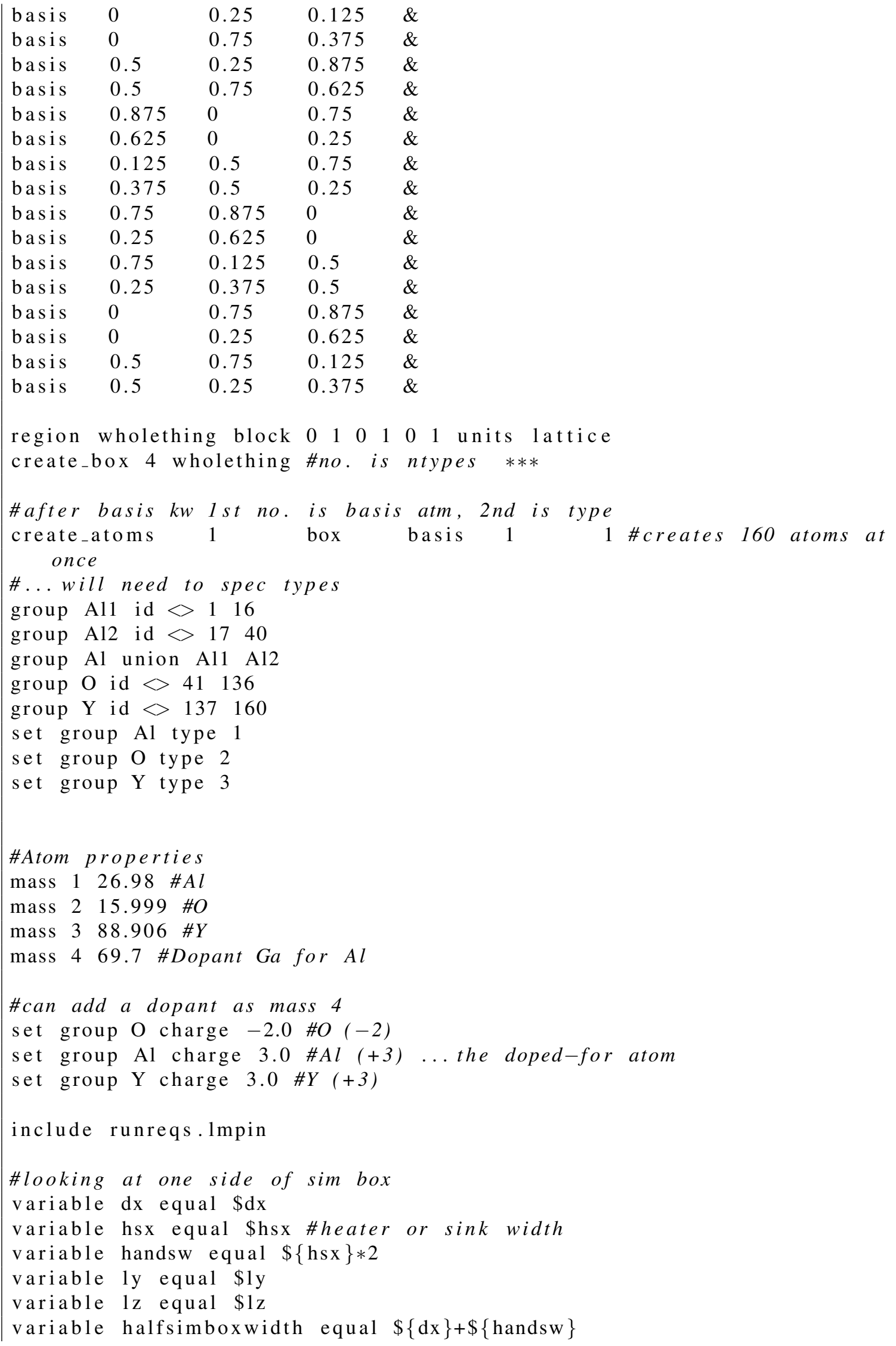




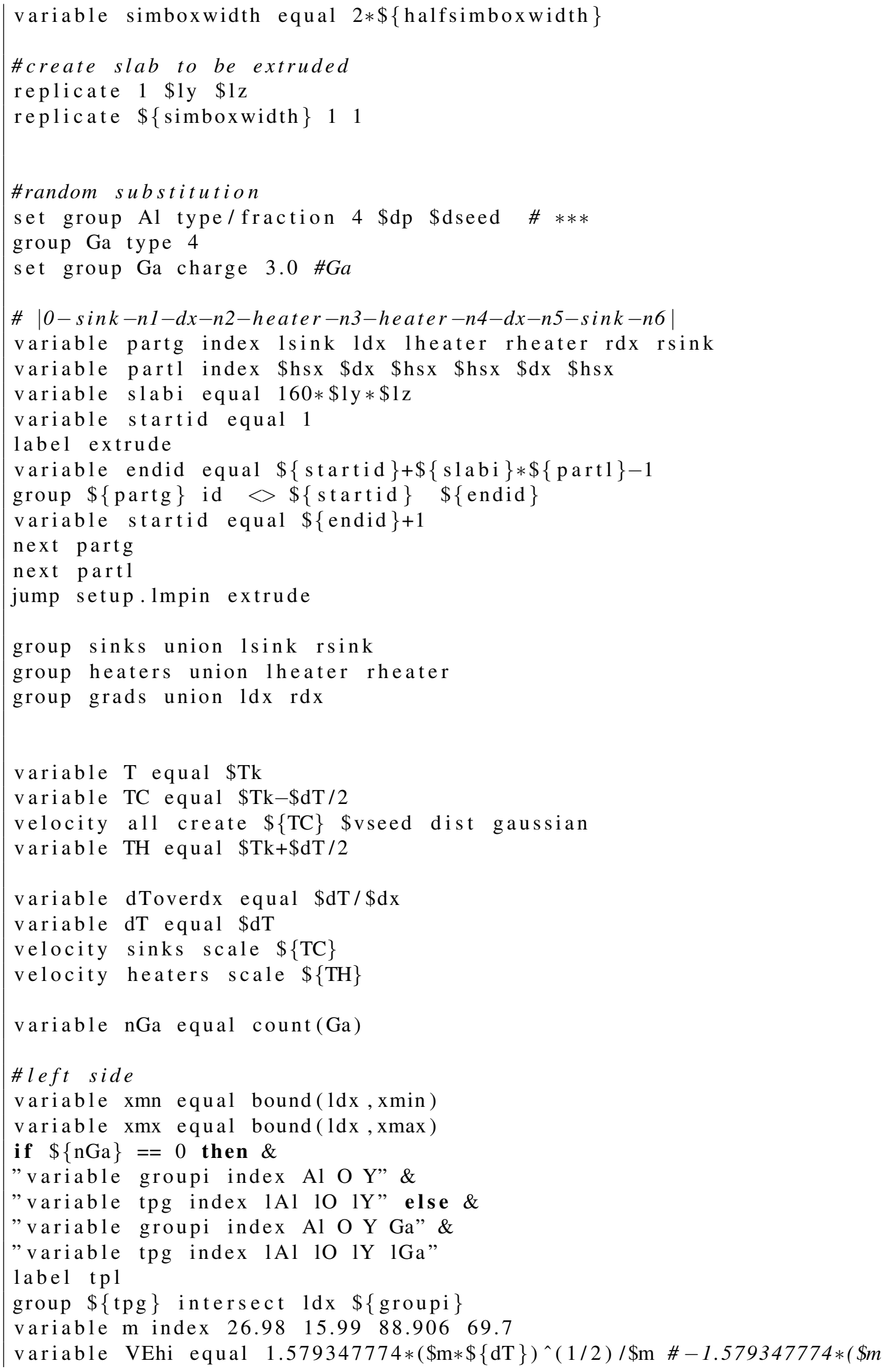




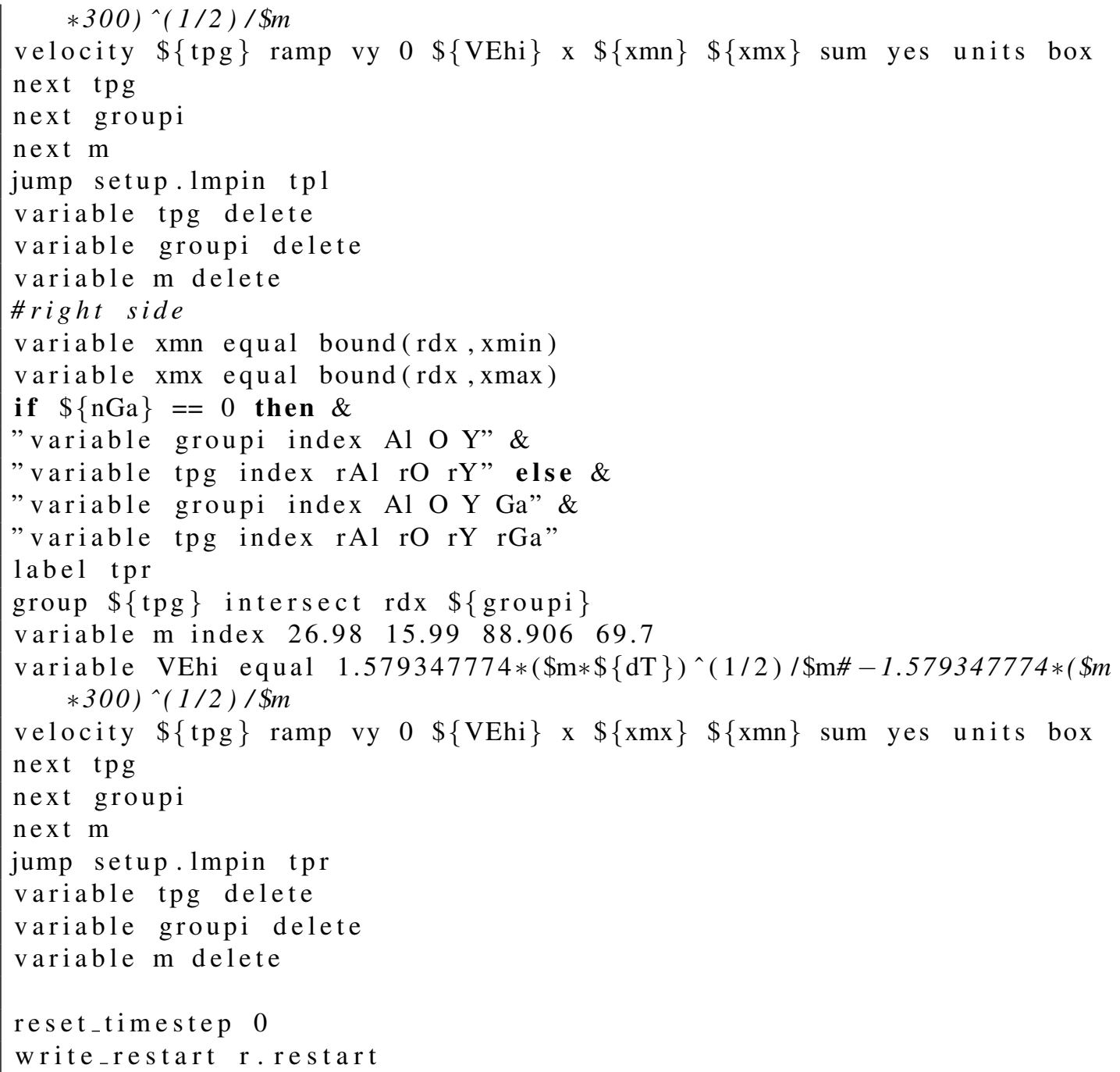

\section{Interatomic Potentials}

runreqs.lmpin

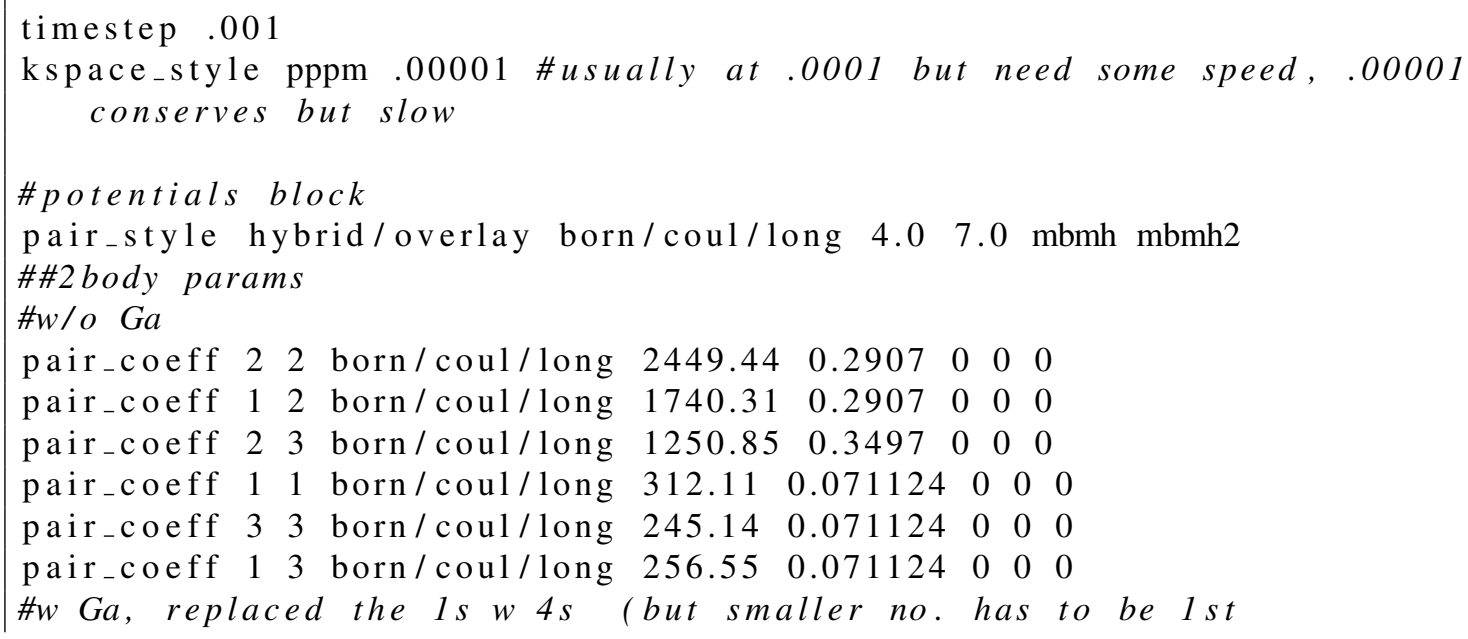


pair_coeff 24 born/coul/long $1740.31 \quad 0.2907 \quad 0 \quad 0 \quad 0$

pair_coeff 44 born/coul/long $312.11 \quad 0.071124 \quad 0 \quad 00$

pair_coeff 14 born/coul/long $312.11 \quad 0.071124 \quad 0 \quad 00$

pair_coeff 34 born/coul/long $256.55 \quad 0.071124 \quad 0 \quad 0 \quad 0$

\#\#3body params

pair_coeff $* *$ mbmh/yag/commoninput/potentials/0/yag.mbmh A O Y A \#maps 4 th type to $A$

pair_coeff $* *$ mbmh2/yag/commoninput/potentials/0/yag.mbmh2 A O Y A

\# recovery from a binary w/ different no of procs

neigh_modify delay 0 every 1 check yes

3-Body parameters (yag.mbmh)

\#3body only for certain kinds and only for XYY

\# $(a *(\operatorname{sigma}=1))$ is rij0, lambda*(epsilon=1) is just lambda

\# KEEP IT 1 FOR ALL EVEN IF NO THREE BODY INTERACTION!

$\# b / c$ same param used in 2 body and we dont want to zero that

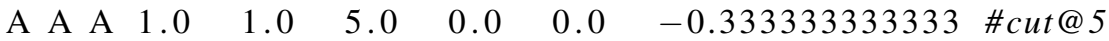

$\begin{array}{lllll}312.11 & 14.06 & 3.0 & 3.0 & 0.0\end{array}$

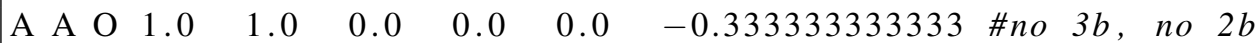

$\begin{array}{lllll}0.0 & 0.0 & 0.0 & 0.0 & 0.0\end{array}$

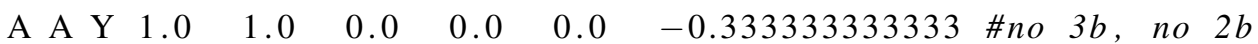
$\begin{array}{lllll}0.0 & 0.0 & 0.0 & 0.0 & 0.0\end{array}$

A O A $1.0 \quad 1.0 \quad 0.0 \quad 0.0 \quad 0.0 \quad-0.333333333333$ \#no $3 b$, no $2 b$ $\begin{array}{lllll}0.0 & 0.0 & 0.0 & 0.0 & 0.0\end{array}$

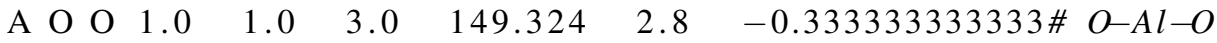

$\begin{array}{lllll}1740.31 & 3.44 & -2.0 & 3.0 & 0.0\end{array}$

A O Y $1.0 \quad 1.0 \quad 0.0 \quad 0.0 \quad 0.0 \quad-0.333333333333$ \#no $3 b$, no $2 b$ $\begin{array}{lllll}0.0 & 0.0 & 0.0 & 0.0 & 0.0\end{array}$

A Y A $1.0 \quad 1.0 \quad 0.0 \quad 0.0 \quad 0.0 \quad-0.333333333333$ \#no $3 b$, no $2 b$ $\begin{array}{lllll}0.0 & 0.0 & 0.0 & 0.0 & 0.0\end{array}$

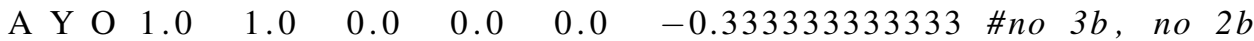
$\begin{array}{lllll}0.0 & 0.0 & 0.0 & 0.0 & 0.0\end{array}$

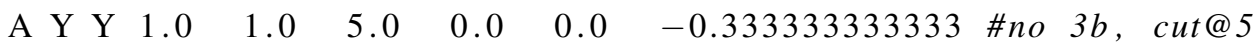
$\begin{array}{lllll}256.55 & 14.06 & 3.0 & 3.0 & 0.0\end{array}$

O A A $1.0 \quad 1.0 \quad 3.0 \quad 0.0 \quad 0.0 \quad-0.333333333333 \quad$ \# $b$ in ind file $\begin{array}{lllll}1740.31 & 3.44 & -2.0 & 3.0 & 0.0\end{array}$

$\begin{array}{llllllllll}\mathrm{O} & \mathrm{A} & \mathrm{O} & 1.0 & 1.0 & 0.0 & 0.0 & 0.0 & -0.333333333333\end{array}$ \#no $3 b$, no $2 b$ $\begin{array}{lllll}0.0 & 0.0 & 0.0 & 0.0 & 0.0\end{array}$

$\mathrm{O}$ A Y $1.0 \quad 1.0 \quad 0.0 \quad 0.0 \quad 0.0 \quad-0.333333333333 \quad$ \#3b in 2 nd file, no $2 b$ $\begin{array}{lllll}0.0 & 0.0 & 0.0 & 0.0 & 0.0\end{array}$

O O A $1.0 \quad 1.0 \quad 0.0 \quad 0.0 \quad 0.0 \quad-0.333333333333$ \#no $3 b$, no $2 b$ $\begin{array}{lllll}0.0 & 0.0 & 0.0 & 0.0 & 0.0\end{array}$

$\begin{array}{llllllll}\text { O O O } & 1.0 & 1.0 & 3.0 & 0.0 & 0.0 & -0.333333333333 & \text { \#cut@3 }\end{array}$ $2449.44 \quad 3.44 \quad-2.0 \quad-2.0 \quad 0.0$

$\begin{array}{llllllllll}\mathrm{O} O \mathrm{Y} & 1.0 & 1.0 & 0.0 & 0.0 & 0.0 & -0.333333333333 & \text { \#no } 3 b, \text { no } 2 b\end{array}$ $\begin{array}{lllll}0.0 & 0.0 & 0.0 & 0.0 & 0.0\end{array}$

$\mathrm{O} Y \mathrm{Y} \quad 1.0 \quad 1.0 \quad 0.0 \quad 0.0 \quad 0.0 \quad-0.333333333333$ \#3b in 2nd file, no $2 b$ $\begin{array}{lllll}0.0 & 0.0 & 0.0 & 0.0 & 0.0\end{array}$

$\begin{array}{llllllllll}\mathrm{O} & \mathrm{Y} & \mathrm{O} & 1.0 & 1.0 & 0.0 & 0.0 & 0.0 & -0.333333333333 & \text { \#no } 3 b, \text { no } 2 b\end{array}$ $\begin{array}{lllll}0.0 & 0.0 & 0.0 & 0.0 & 0.0\end{array}$

O Y Y $1.0 \quad 1.0 \quad 3.0 \quad 0.0 \quad 0.0 \quad-0.333333333333 \quad$ \#3b in 2nd file $\begin{array}{lllll}1250.85 & 2.86 & -2.0 & 3.0 & 0.0\end{array}$ 


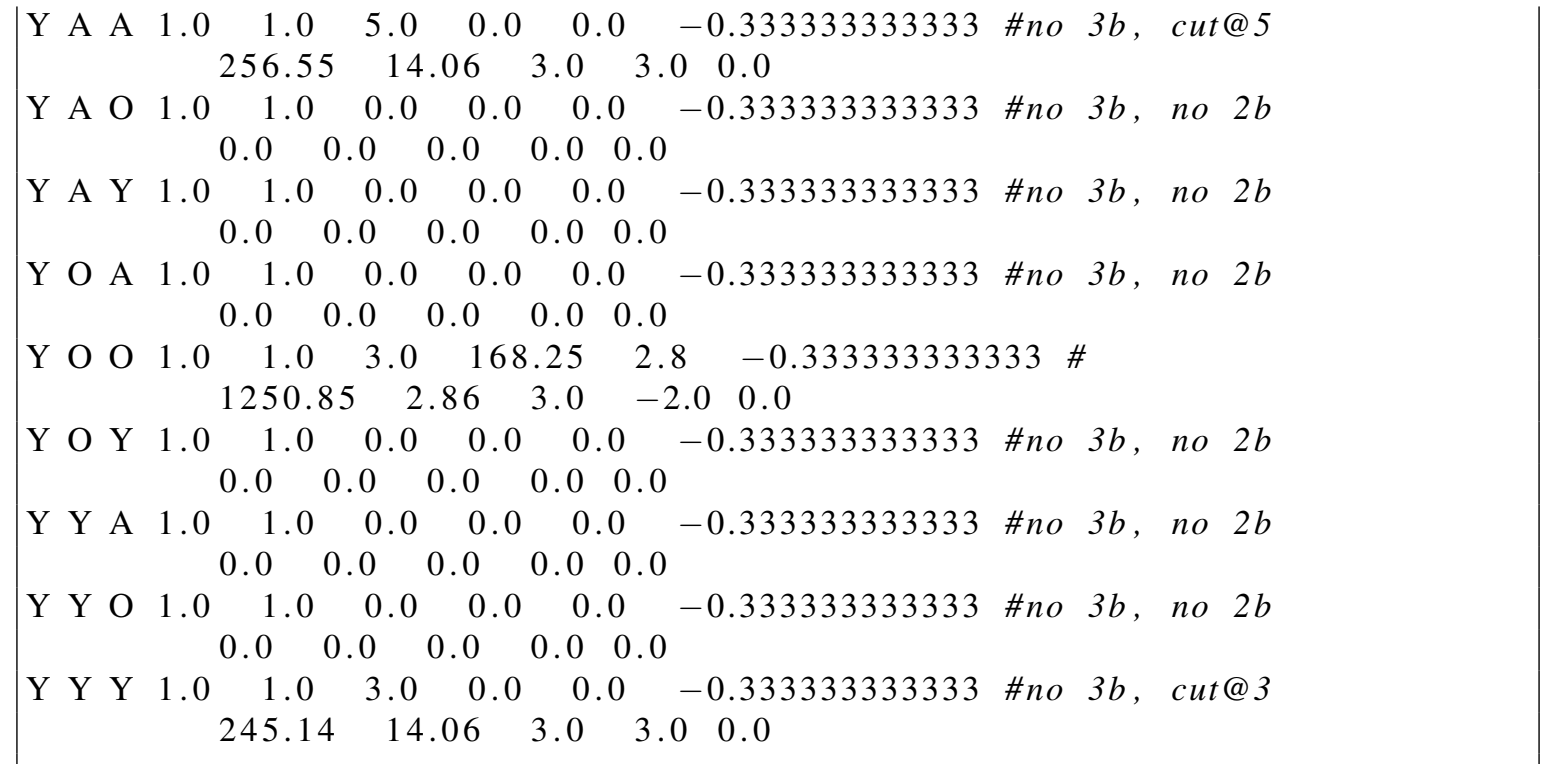

\section{3-Body parameters (yag.mbmh2)}

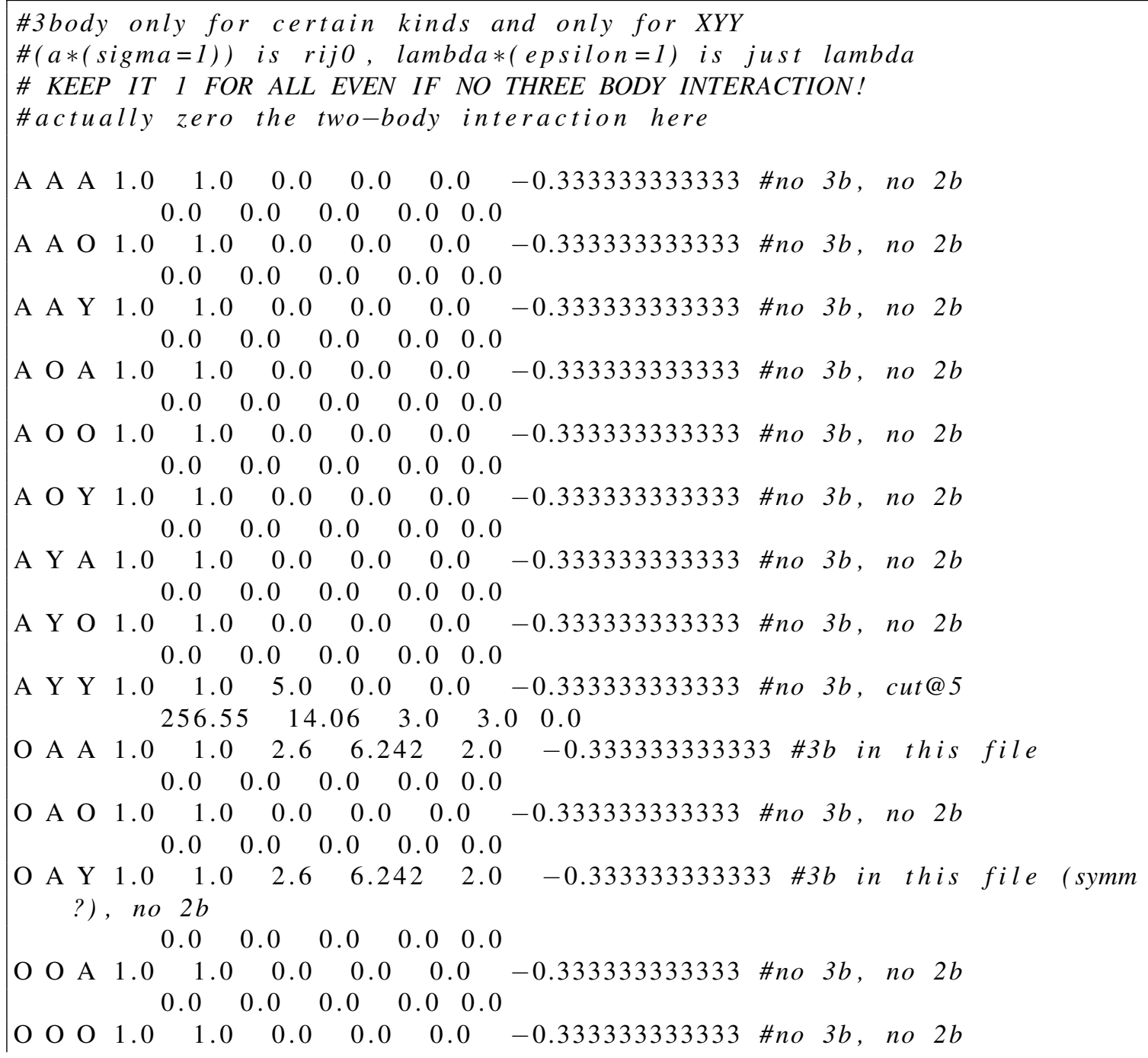




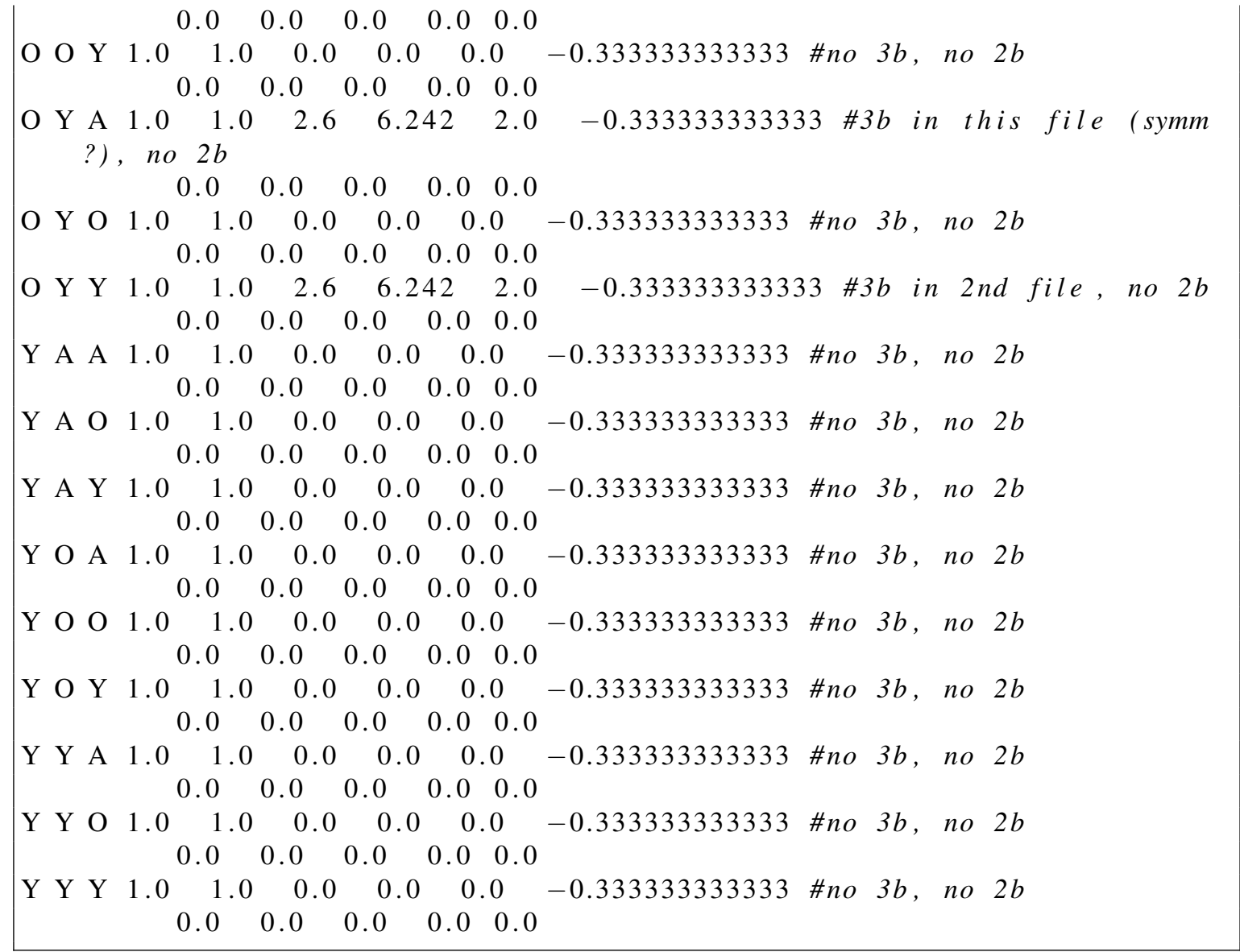

\section{Simulation Execution}

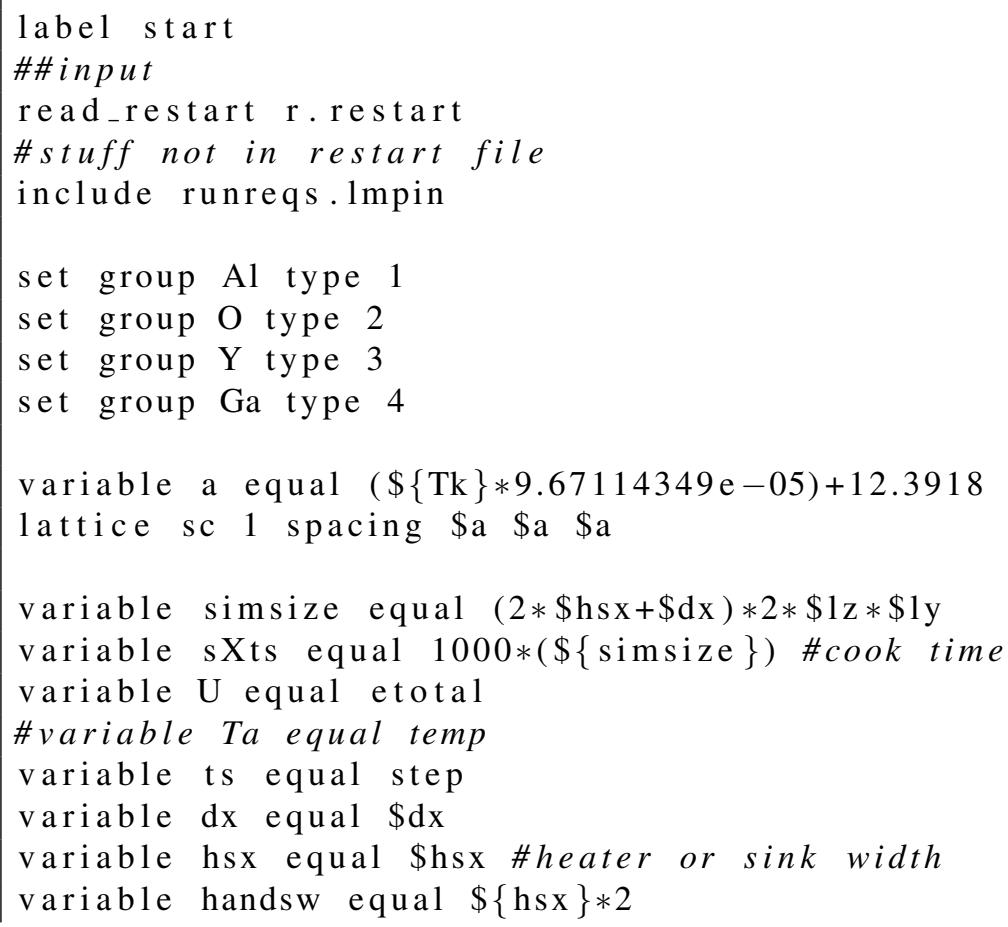




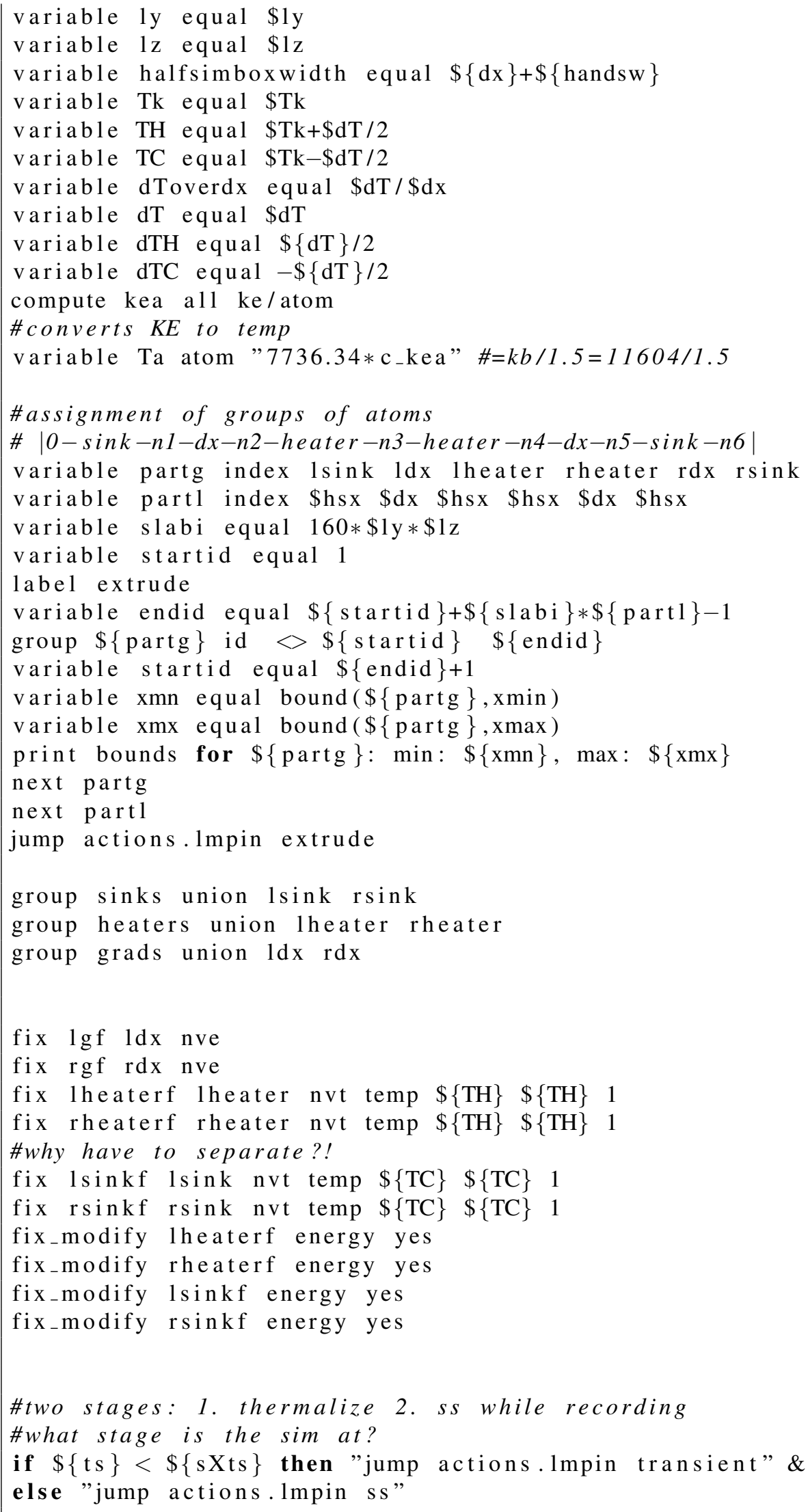




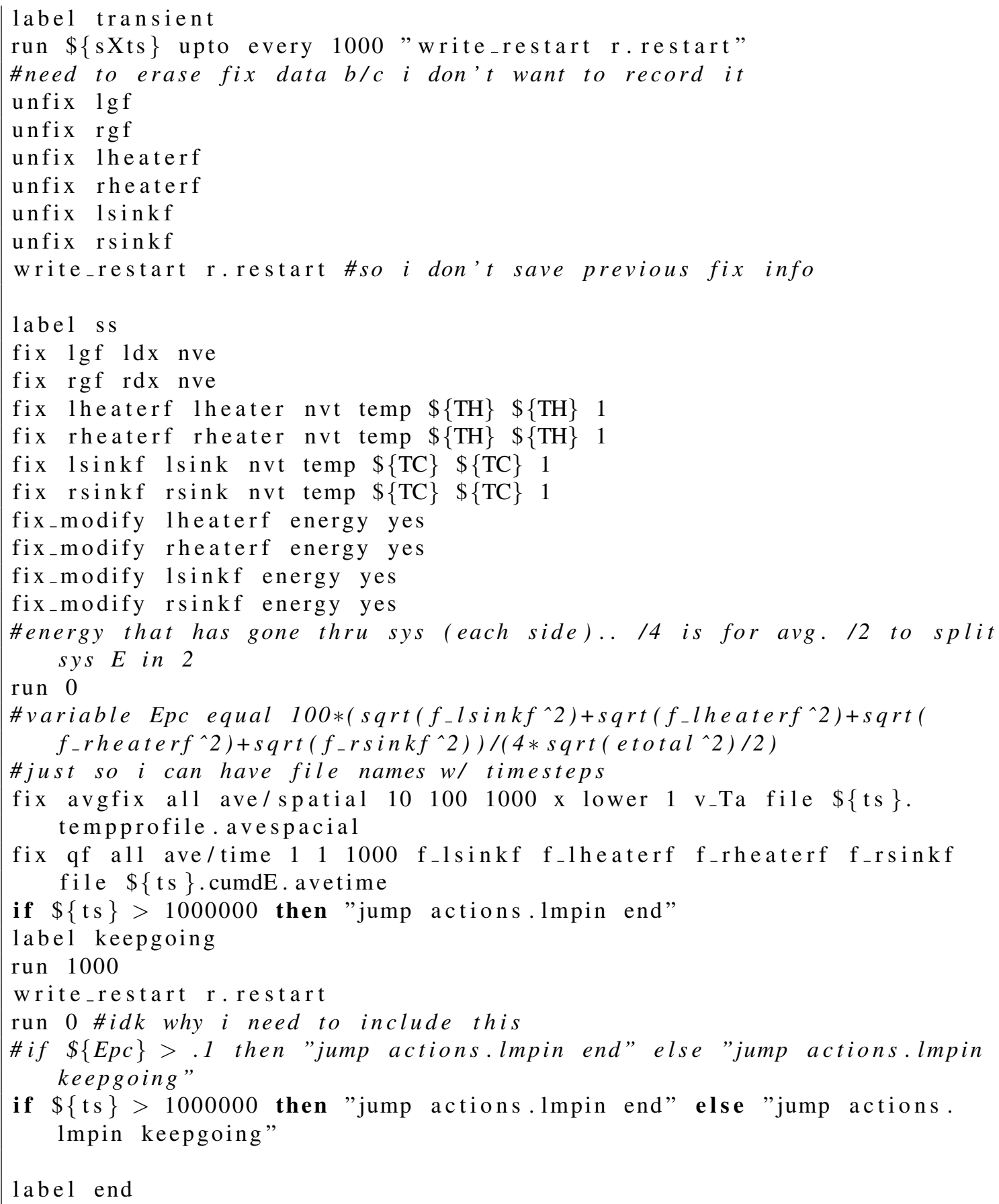




\section{BIBLIOGRAPHY}

[1] "Jmol: an open-source java viewer for chemical structures in 3d," On the WWW, URL http: //www . jmol.org/.

[2] L. Dobrzycki, E. Bulska, D. A. Pawlak, Z. Frukacz, and K. Wozniak, "Structure of YAG crystals doped/substituted with erbium and ytterbium," Inorganic Chemistry, vol. 43, no. 24, pp. 7656-7664, 2004, pMID: 15554630.

[3] A. Potdevin, G. Chadeyron, and R. Mahiou, " $\mathrm{Tb}^{3+}$-doped yttrium garnets: Promising tunable green phosphors for solid-state lighting," Chemical Physics Letters, vol. 490, no. $1-3$, pp. $50-53,2010$.

[4] R. Hansel, S. Allison, and G. Walker, "Temperature-dependent luminescence of gallium-substituted YAG:Ce," Journal of Materials Science, vol. 45, pp. 146-150, 2010, 10.1007/s10853-009-3906-9.

[5] X. Q. Cao, R. Vassen, and D. Stoever, "Ceramic materials for thermal barrier coatings," Journal of the European Ceramic Society, vol. 24, no. 1, pp. 1 - 10, 2004.

[6] A. Ikesue, Y. L. Aung, T. Taira, T. Kamimura, K. Yoshida, and G. L. Messing, "Progress in ceramic lasers," Annual Review of Materials Research, vol. 36, no. 1, pp. 397-429, 2006.

[7] K. Sattler, Handbook of Nanophysics: Nanoparticles and Quantum Dots, ser. Handbook of Nanophysics.

[8] J. L. Caslavsky and D. J. Viechnicki, "Melting behaviour and metastability of yttrium aluminium garnet (YAG) and $\mathrm{YAlO}_{3}$ determined by optical differential thermal analysis," Journal of Materials Science, vol. 15, pp. 1709-1718, 1980, 10.1007/BF00550589.

[9] R. Wynne, J. L. Daneu, and T. Y. Fan, "Thermal coefficients of the expansion and refractive index in YAG," Appl. Opt., vol. 38, no. 15, pp. 3282-3284, 1999.

[10] Red Optronics, "Undoped yag crystal $\left(\mathrm{Y}_{3} \mathrm{Al}_{5} \mathrm{O}_{12}\right)$," On the WWW, URL http://www . redoptronics.com/undoped-YAG-crystal.html.

[11] H. Qiu, P. Yang, J. Dong, P. Deng, J. Xu, and W. Chen, "The influence of Yb concentration on laser crystal Yb:YAG," Materials Letters, vol. 55, no. 1-2, pp. 1 - 7, 2002.

[12] J. Morikawa, C. Leong, T. Hashimoto, T. Ogawa, Y. Urata, S. Wada, M. Higuchi, and J. ichi Takahashi, "Thermal conductivity/diffusivity of $\mathrm{Nd}^{3+}$ doped $\mathrm{GdVO}_{4}, \mathrm{YVO}_{4}$, $\mathrm{LuVO}_{4}$, and $\mathrm{Y}_{3} \mathrm{Al}_{5} \mathrm{O}_{12}$ by temperature wave analysis," Journal of Applied Physics, vol. 103 , no. 6 , p. 063522, 2008.

[13] J. F. W. Gunsser, U. Wolfmeier, 1.3.8.4 Debye Temperature, A. M. H. K.-H. Hellwege, Ed. Springer-Verlag, 1978, vol. 12a. 
[14] J. Bodzenta, A. Kaźmierczak-Bałata, K. B. Wokulska, J. Kucytowski, T. Łukasiewicz, and W. Hofman, "Influence of doping on thermal diffusivity of single crystals used in photonics: measurements based on thermal wave methods," Appl. Opt., vol. 48, no. 7, pp. C46-C54, 2009.

[15] C. Jun, C. Dong-Quan, and Z. Jing-Lin, "Molecular dynamics simulation of thermodynamic properties of YAG," Chinese Physics, vol. 16, no. 9, p. 2779, 2007.

[16] S. Plimpton, "Fast parallel algorithms for short-range molecular-dynamics," Journal of Computational Physics, vol. 117, no. 1, pp. 1-19, March 11995.

[17] L. Verlet, "Computer "Experiments" on Classical Fluids. II. Equilibrium Correlation Functions," Phys. Rev., vol. 165, no. 1, pp. 201-214, Jan 1968.

[18] M. P. Tosi and F. G. Fumi, "Ionic sizes and born repulsive parameters in the NaCltype alkali halides-II : The generalized Huggins-Mayer form," Journal of Physics and Chemistry of Solids, vol. 25, no. 1, pp. 45 - 52, 1964.

[19] Z. Phys., vol. 11.

[20] M. E. Glicksman, Principles of Solidification : an introduction to modern casting and crystal growth concepts. New York, New York: Springer, 2011, ch. 1, p. 17.

[21] J. G. O. Ojwang, R. van Santen, G. J. Kramer, A. C. T. van Duin, and I. W. A. Goddard, "Predictions of melting, crystallization, and local atomic arrangements of aluminum clusters using a reactive force field," vol. 129, no. 24, p. 244506, 2008.

[22] D. P. Sellan, E. S. Landry, J. E. Turney, A. J. H. McGaughey, and C. H. Amon, Phys. Rev. $B$, vol. 81, Jun. 